\title{
SWIFT X-RAY OBSERVATIONS OF CLASSICAL NOVAE. II. THE SUPER SOFT SOURCE SAMPLE
}

\author{
Greg J. Schwarz ${ }^{1}$, Jan-Uwe Ness ${ }^{2}$, J. P. Osborne ${ }^{3}$, K. L. Page ${ }^{3}$, P. A. Evans ${ }^{3}$, A. P. Beardmore ${ }^{3}$, Frederick M. Walter ${ }^{4}$, \\ L. Andrew Helton ${ }^{5}$, Charles E. Woodward ${ }^{6}$, Mike Bode ${ }^{7}$, Sumner Starrfield ${ }^{8}$, and Jeremy J. Drake ${ }^{9}$ \\ ${ }^{1}$ American Astronomical Society, 2000 Florida Avenue, NW, Suite 400, Washington, DC 20009-1231, USA; Greg.Schwarz@ aas.org \\ ${ }^{2}$ XMM-Newton Science Operations Centre, ESAC, Apartado 78, 28691 Villanueva de la Canada, Madrid, Spain \\ ${ }^{3}$ Department of Physics and Astronomy, University of Leicester, Leicester LE1 7RH, UK \\ ${ }^{4}$ Department of Physics and Astronomy, Stony Brook University, Stony Brook, NY 11794-3800, USA \\ ${ }^{5}$ SOFIA Science Center, USRA, NASA Ames Research Center, M.S. N211-3, Moffett Field, CA 94035, USA \\ ${ }^{6}$ Minnesota Institute of Astrophysics, 116 Church Street S.E., University of Minnesota, Minneapolis, MN 55455, USA \\ ${ }^{7}$ Astrophysics Research Institute, Liverpool John Moores University, Birkenhead CH41 1LD, UK \\ ${ }^{8}$ School of Earth and Space Exploration, Arizona State University, P.O. Box 871404, Tempe, AZ 85287-1404, USA \\ ${ }^{9}$ Smithsonian Astrophysical Observatory, 60 Garden Street, MS 3, Cambridge, MA 02138, USA \\ Received 2011 April 13; accepted 2011 October 27; published 2011 November 29
}

\begin{abstract}
The Swift gamma-ray burst satellite is an excellent facility for studying novae. Its rapid response time and sensitive $\mathrm{X}$-ray detector provides an unparalleled opportunity to investigate the previously poorly sampled evolution of novae in the X-ray regime. This paper presents Swift observations of 52 Galactic/Magellanic Cloud novae. We included the X-Ray Telescope (0.3-10 keV) instrument count rates and the UltraViolet and Optical Telescope (1700-8000 A) filter photometry. Also included in the analysis are the publicly available pointed observations of 10 additional novae the X-ray archives. This is the largest X-ray sample of Galactic/Magellanic Cloud novae yet assembled and consists of 26 novae with Super Soft X-ray emission, 19 from Swift observations. The data set shows that the faster novae have an early hard X-ray phase that is usually missing in slower novae. The Super Soft X-ray phase occurs earlier and does not last as long in fast novae compared to slower novae. All the Swift novae with sufficient observations show that novae are highly variable with rapid variability and different periodicities. In the majority of cases, nuclear burning ceases less than three years after the outburst begins. Previous relationships, such as the nuclear burning duration versus $t_{2}$ or the expansion velocity of the eject and nuclear burning duration versus the orbital period, are shown to be poorly correlated with the full sample indicating that additional factors beyond the white dwarf mass and binary separation play important roles in the evolution of a nova outburst. Finally, we confirm two optical phenomena that are correlated with strong, soft X-ray emission which can be used to further increase the efficiency of X-ray campaigns.
\end{abstract}

Key words: novae, cataclysmic variables - ultraviolet: stars - X-rays: stars

Online-only material: figure set, machine-readable table

\section{INTRODUCTION}

Novae occur in binary systems in which a Roche lobe filling secondary is losing hydrogen-rich material through the inner Lagrangian point onto a white dwarf (WD) primary. Mass transfer can also occur in long-period systems if the secondary has a significant wind, e.g., the giant secondary in RS Oph or V407 Cyg. Core material is mixed into the accreted material and is violently ejected into space when the pressure at the WD-accretion interface becomes great enough to initiate a thermonuclear runaway (TNR). Novae eject, into the interstellar medium (ISM), a mixture of material accreted from the companion star, highly processed material from the underlying WD, and products of nucleosynthesis occurring during the TNR. As a result of the TNR, up to $10^{-4} M_{\odot}$ of material can be ejected from the WD enriched in $\mathrm{C}, \mathrm{N}, \mathrm{O}, \mathrm{Ne}, \mathrm{Mg}, \mathrm{Al}$, and other species (José et al. 2006) at $v \sim 10^{2}-10^{4} \mathrm{~km} \mathrm{~s}^{-1}$. Any remaining hydrogen still bound to the WD continues to burn in hydrostatic equilibrium until it is consumed or ejected via a wind.

Initially, the radiative output of a nova occurs in the optical but as the photosphere of the WD recedes, the spectral energy distribution (SED) shifts to higher energies (Gallagher \& Starrfield 1978). The rate of the optical decline defines a nova's primary characteristics (e.g., Warner 2008, and references therein), namely, the time to decline 2 mag from visual maximum, $t_{2}$. The decline rate depends on the amount of mass ejected, its velocity, composition, and if it runs into circumbinary material. The bolometric luminosity during the outburst is high, near or exceeding the Eddington limit (for the fastest novae), and thus additional material is ejected via a strong stellar wind (Schwarz et al. 1998, 2001). In some novae the collision between this fast wind and the initial exploded mass or any pre-existing circumbinary material can produce $\mathrm{X}$-ray emission from shocks. The emission from this early X-ray phase is hard, has a low luminosity, of order $10^{33}-10^{35} \mathrm{erg} \mathrm{s}^{-1}$, and declines relatively rapidly (Balman et al. 1998; Orio et al. 2001b). As fuel continues to burn, mass loss causes the photosphere of the WD to shrink (MacDonald et al. 1985). The effective temperature increases, peaking in the soft X-rays, at $(2-8) \times 10^{5} \mathrm{~K}$ (Krautter et al. 1996; Shore et al. 1996; Rauch et al. 2010). Once the ejecta have cleared sufficiently, and if the line of sight extinction is not severe, some novae exhibit characteristics similar to the Super Soft X-ray binary sources (SSSs; Kahabka \& van den Heuvel 1997 ) with strong and soft, $E_{\text {peak }}<1 \mathrm{keV}$, X-ray emission. This point in novae evolution is called the SSS phase. At low spectral resolution, the UV/X-ray SEDs resemble blackbodies, but higher resolution Chandra or XMM grating observations 
reveal a significantly more complex picture. The spectra frequently have P-Cygni profiles or emission lines superimposed on a line blanketed atmosphere. Models sophisticated enough to interpret the high-resolution data are only now becoming available (van Rossum \& Ness 2010). Once nuclear burning ends, the X-ray light curve rapidly declines as the WD cools marking the end of the SSS phase and the outburst. At some point mass transfer resumes and eventually another eruption occurs. These are called classical novae $(\mathrm{CNe})$ until a second outburst is observed then they become recurrent novae ( $\mathrm{RNe})$. Detailed reviews of nova evolution are presented by Starrfield et al. (2008) and Bode (2010). Hernanz \& Sala (2010) discuss the theoretical implications of X-ray observations of novae, while Schaefer (2010) discusses the current understanding of the RN class.

An important, but not the sole driver of the nova phenomenon is the mass of the WD. Explosions on larger mass WDs expel less mass but at higher velocities. They have larger luminosities, are in outburst for less time, and (should) have shorter recurrence times than novae on lower mass WDs. Highmass $\left(>1.25 M_{\odot}\right)$ WDs reach TNR ignition more rapidly than low-mass WDs and thus do not have the chance to accrete as much material. They also reach higher peak temperatures during the TNR leading to a more energetic explosion. However, other factors are believed to play important roles leading to a nova event. These include the composition of the WD, either $\mathrm{CO}$ or $\mathrm{ONe}$, the initial temperature of the WD during accretion, the mass accretion rate (Yaron et al. 2005), the composition of the accreted material (Starrfield et al. 2000), and the mixing history of the core/envelope. All impact how much mass can be transferred to the WD before an outburst begins. Models show that different combinations of these characteristics can reproduce a wide range of nova outbursts (Yaron et al. 2005; Woodward \& Starrfield 2011). Unfortunately, very few of these parameters have been observationally verified in any nova.

The X-ray regime is a crucial component for the study of novae providing insight into TNR burning processes, WD mass and composition, accretion and mixing mechanisms, dust grain formation and destruction, and mass-loss processes. In addition, high-mass novae such as RNe are potential SN Ia progenitors via the single degenerate scenario (e.g., Walder et al. 2008, 2010; Meng \& Yang 2010). To make progress understanding the physics of these important astrophysical phenomena, observations of a large number of novae are required to sample all the contributing factors. Prior to the launch of Swift the general X-ray temporal evolution of novae was far from complete as only a few novae had been observed at more than one epoch in X-rays.

Swift is an excellent facility for studying novae as it has a superb soft X-ray response with its X-Ray Telescope (XRT) instrument (Burrows et al. 2005). Ness et al. (2007) show how the XRT favorably compares with currently available X-ray instruments. Swift also has a co-aligned UV/optical instrument, the UltraViolet and Optical Telescope (UVOT; see Roming et al. 2005 for details), which provides either six filter photometry or low-resolution grism spectroscopy. The other Swift instrument is a $\gamma$-ray detector, BAT. However, novae are generally not strong $\gamma$-ray sources (Hernanz \& José 2005). The decay of ${ }^{22} \mathrm{Na}$ (half-life 2.6 years) generates a $1275 \mathrm{keV}$ emission line but only $>1.25 M_{\odot}$ WDs are predicted to produce sufficient ${ }^{22} \mathrm{Na}$ during the TNR. This line has not yet been definitively detected by satellites (Hernanz 2008) but there is a recent claim by Suzuki \& Shigeyama (2010) that their models with Compton decay of ${ }^{22} \mathrm{Na}$ can account for the hard X-ray flux in V2491 Cyg provided an exceptionally large amount of ${ }^{22} \mathrm{Na}, 3 \times$ $10^{-5} M_{\odot}$, was synthesized. Another $\gamma$-ray emission mechanism is electron-positron annihilation very early in the outburst but this is expected to be detectable only in nearby novae. The symbiotic RN RS Oph, at $1.6 \mathrm{kpc}$, was clearly detected in the lowest energy channels of the Swift/Burst Alert Telescope (Senziani et al. 2008), but that emission is consistent with that from high temperature shocks as the outburst ejecta plow into the pre-existing red giant wind. Recently the symbiotic RN V407 Cyg was detected in the $\mathrm{GeV}$ band by Fermi Large Area Telescope (LAT; Abdo et al. 2010) only a few days after visual maximum. Abdo et al. (2010) show that the $\gamma$-ray emission can be explained by either Compton scattering of infrared photons in the red giant wind or $\pi^{0}$ decay from proton-proton collisions. Lu et al. (2011) predict that $\pi^{0} \gamma$-rays will be created in the high circumbinary densities of very long orbital periods systems such as V407 Cyg, with a period of $\sim 43$ years (Munari et al. 1990; Shore et al. 2011).

Swift has a rapid response target of opportunity (ToO) procedure and flexible scheduling, which is critical in obtaining well-sampled X-ray light curves of transient events. Initial Swift results of 11 novae were presented in Ness et al. (2007). Since that time significantly more data have been obtained by the Swift Nova-CV group, ${ }^{10}$ which has devised an observing strategy to efficiently utilize the satellite's unique capabilities and maximize the science return by observing interesting and bright novae with low extinction recently discovered in the Milky Way and Magellanic Clouds. In five years Swift has performed multiple visits for 52 classical and recurrent Galactic/Magellanic Cloud novae totaling well over $2 \mathrm{Ms}$ of exposure time.

Here we present a summary of all the Galactic/Magellanic Cloud Swift nova observations from launch (2004 November $20)$ to 2010 July 31 using the XRT (0.3-10 keV) X-ray instrument (count rates and hardness ratios) and the available UVOT (1700-8000 A) filter photometry. Swift observations of novae in the M31 group are reported in Henze et al. (2010), Pietsch (2010), and references within. We combine the Swift Galactic/Magellanic Cloud data with archival pointed observations of $\mathrm{CNe}$ and RNe from ROSAT, XMM, Chandra, BeppoSAX, RXTE, and ASCA to produce the most comprehensive $X$-ray sample of local nova. The sample includes 26 systems that were observed during the SSS phase.

In Section 2, we summarize the properties of the 62 novae in the X-ray sample. The averaged Swift XRT count rates and UVOT magnitudes for each observational session are also provided. Studies of high-frequency phenomena in individual objects are either left for future work or have previously been published (V458 Vul, V2941 Cyg, V598 Pup, RS Oph, V407 Cyg, and V723 Cas in Ness et al. 2008a, 2009a; Page et al. 2010, 2009; Osborne et al. 2011; Shore et al. 2011, respectively). Sections 3 and 4 detail the observations and results during the hard and SSS phases, respectively. A discussion follows in Section 5 articulating trends between the SSS duration and $t_{2}$, expansion velocity of the ejecta, and orbital period plus the role of SSS emission in dust-forming novae. Also included is a discussion on the origin of the different variability observed in the X-ray and UV light curves of the Swift sources. Optical characteristics indicative of SSS emission in $\mathrm{CN}$ and $\mathrm{RN}$ are

\footnotetext{
10 The current members of the group and observation strategy are provided at
} http://www.swift.ac.uk/nova-cv/. 
Table 1

Observable Characteristics of Galactic/Magellanic Cloud Novae with X-Ray Observations

\begin{tabular}{|c|c|c|c|c|c|c|c|c|c|c|}
\hline Name $^{a}$ & $\begin{array}{l}V_{\max }^{\mathrm{b}} \\
(\mathrm{mag})\end{array}$ & $\begin{array}{c}\text { Date }^{c} \\
\text { (JD) }\end{array}$ & $\begin{array}{c}t_{2}{ }^{\mathrm{d}} \\
\text { (days) }\end{array}$ & $\begin{array}{l}\mathrm{FWHM}^{\mathrm{e}} \\
\left(\mathrm{km} \mathrm{s}^{-1}\right)\end{array}$ & $\begin{array}{c}E(B-V) \\
(\mathrm{mag})\end{array}$ & $\begin{array}{c}N_{\mathrm{H}}{ }^{\mathrm{f}} \\
\left(\mathrm{cm}^{-2}\right)\end{array}$ & $\begin{array}{l}\text { Period } \\
\text { (days) }\end{array}$ & $\begin{array}{c}D \\
(\mathrm{kpc})\end{array}$ & Dust?g & RN? \\
\hline CI Aql & $8.83(1)$ & $2451665.5(1)$ & $32(2)$ & $2300(3)$ & $0.8 \pm 0.2(4)$ & $1.2 \mathrm{e}+22$ & $0.62(4)$ & $6.25 \pm 5(4)$ & $\mathrm{N}$ & $\mathrm{Y}$ \\
\hline $\operatorname{CSS081007}^{\mathrm{h}}$ & $\ldots$ & $2454596.5^{\mathrm{i}}$ & $\ldots$ & $\ldots$ & 0.146 & $1.1 \mathrm{e}+21$ & $1.77(5)$ & $4.45 \pm 1.95(6)$ & $\ldots$ & $\ldots$ \\
\hline GQ Mus & $7.2(7)$ & $2445352.5(7)$ & $18(7)$ & $1000(8)$ & $0.45(9)$ & $3.8 e+21$ & $0.059375(10)$ & $4.8 \pm 1(9)$ & $\mathrm{N}(7)$ & $\ldots$ \\
\hline IM Nor & $7.84(11)$ & $2452289(2)$ & $50(2)$ & $1150(12)$ & $0.8 \pm 0.2(4)$ & $8 e+21$ & $0.102(13)$ & $4.25 \pm 3.4(4)$ & $\mathrm{N}$ & $\mathrm{Y}$ \\
\hline KT Eri & $5.42(14)$ & $2455150.17(14)$ & $6.6(14)$ & $3000(15)$ & $0.08(15)$ & $5.5 e+20$ & $\ldots$ & $6.5(15)$ & $\mathrm{N}$ & M \\
\hline LMC 1995 & $10.7(16)$ & $2449778.5(16)$ & $15 \pm 2(17)$ & $\ldots$ & $0.15(203)$ & $7.8 e+20$ & . & 50 & $\ldots$ & $\ldots$ \\
\hline LMC 2000 & $11.45(18)$ & $2451737.5(18)$ & $9 \pm 2(19)$ & $1700(20)$ & $0.15(203)$ & $7.8 \mathrm{e}+20$ & $\ldots$ & 50 & $\ldots$ & $\ldots$ \\
\hline LMC 2005 & $11.5(21)$ & $2453700.5(21)$ & $63(22)$ & $900(23)$ & $0.15(203)$ & $1 e+21$ & $\ldots$ & 50 & M (24) & $\ldots$ \\
\hline LMC 2009a & $10.6(25)$ & $2454867.5(25)$ & $4 \pm 1$ & $3900(25)$ & $0.15(203)$ & $5.7 e+20$ & $1.19(26)$ & 50 & $\mathrm{~N}$ & $\mathrm{Y}$ \\
\hline SMC 2005 & $10.4(27)$ & $2453588.5(27)$ & $\ldots$ & $3200(28)$ & $\ldots$ & $5 e+20$ & $\ldots$ & 61 & $\ldots$ & $\ldots$ \\
\hline QY Mus & $8.1(29)$ & $2454739.90(29)$ & 60: & $\ldots$ & $0.71(30)$ & $4.2 e+21$ & $\ldots$ & $\ldots$ & M & $\ldots$ \\
\hline RS Oph & $4.5(31)$ & $2453779.44(14)$ & $7.9(14)$ & $3930(31)$ & $0.73(32)$ & $2.25 \mathrm{e}+21$ & $456(33)$ & $1.6 \pm 0.3(33)$ & $\mathrm{N}(34)$ & $\mathrm{Y}$ \\
\hline U Sco & $8.05(35)$ & $2455224.94(35)$ & $1.2(36)$ & $7600(37)$ & $0.2 \pm 0.1$ & $1.2 \mathrm{e}+21$ & $1.23056(36)$ & $12 \pm 2(4)$ & $\mathrm{N}$ & $\mathrm{Y}$ \\
\hline V1047 Cen & $8.5(38)$ & $2453614.5(39)$ & $6(40)$ & $840(38)$ & $\ldots$ & $1.4 \mathrm{e}+22$ & $\ldots$ & $\ldots$ & $\ldots$ & $\ldots$ \\
\hline V1065 Cen & $8.2(41)$ & $2454123.5(41)$ & $11(42)$ & $2700(43)$ & $0.5 \pm 0.1(42)$ & $3.75 \mathrm{e}+21$ & $\ldots$ & $9.05 \pm 2.8(42)$ & $\mathrm{Y}(42)$ & $\ldots$ \\
\hline V1187 Sco & $7.4(44)$ & $2453220.5(44)$ & $7:(45)$ & $3000(44)$ & $1.56(44)$ & $8.0 \mathrm{e}+21$ & $\ldots$ & $4.9 \pm 0.5(44)$ & $\mathrm{N}$ & $\ldots$ \\
\hline V1188 Sco & $8.7(46)$ & $2453577.5(46)$ & $7(40)$ & $1730(47)$ & $\ldots$ & $5.0 \mathrm{e}+21$ & $\ldots$ & $7.5(39)$ & $\ldots$ & $\ldots$ \\
\hline V1213 Cen & $8.53(48)$ & $2454959.5(48)$ & $11 \pm 2(49)$ & $2300(50)$ & $2.07(30)$ & $1.0 \mathrm{e}+22$ & $\ldots$ & $\ldots$ & $\ldots$ & $\ldots$ \\
\hline V1280 Sco & $3.79(51)$ & 2454147.65 (14) & $21(52)$ & $640(53)$ & $0.36(54)$ & $1.6 e+21$ & $\ldots$ & $1.6 \pm 0.4(54)$ & Y (54) & $\ldots$ \\
\hline V1281 Sco & $8.8(55)$ & $2454152.21(55)$ & 15: & $1800(56)$ & $0.7(57)$ & $3.2 \mathrm{e}+21$ & $\ldots$ & $\ldots$ & $\mathrm{N}$ & $\ldots$ \\
\hline V1309 Sco & $7.1(58)$ & $2454714.5(58)$ & $23 \pm 2(59)$ & $670(60)$ & $1.2(30)$ & $4.0 \mathrm{e}+21$ & $\ldots$ & $\ldots$ & $\ldots$ & $\ldots$ \\
\hline V1494 Aql & $3.8(61)$ & $2451515.5(61)$ & $6.6 \pm 0.5(61)$ & $1200(62)$ & $0.6(63)$ & $3.6 e+21$ & $0.13467(64)$ & $1.6 \pm 0.1(63)$ & $\mathrm{N}$ & $\ldots$ \\
\hline V1663 Aql & $10.5(65)$ & $2453531.5(65)$ & $17(66)$ & $1900(67)$ & $2:(68)$ & $1.6 e+22$ & $\ldots$ & $8.9 \pm 3.6(69)$ & $\mathrm{N}$ & $\ldots$ \\
\hline V1974 Cyg & $4.3(70)$ & $2448654.5(70)$ & $17(71)$ & 2000 (19) & $0.36 \pm 0.04(71)$ & $2.7 e+21$ & $0.081263(70)$ & $1.8 \pm 0.1(72)$ & $\mathrm{N}$ & $\ldots$ \\
\hline V2361 Cyg & $9.3(73)$ & $2453412.5(73)$ & $6(40)$ & $3200(74)$ & 1.2: $(75)$ & $7.0 \mathrm{e}+21$ & $\ldots$ & $\ldots$ & $\mathrm{Y}(40)$ & $\ldots$ \\
\hline V2362 Cyg & $7.8(76)$ & $2453831.5(76)$ & $9(77)$ & $1850(78)$ & $0.575 \pm 0.015(79)$ & $4.4 e+21$ & $0.06577(80)$ & $7.75 \pm 3(77)$ & $\mathrm{Y}(81)$ & $\ldots$ \\
\hline V2467 Cyg & $6.7(82)$ & $2454176.27(82)$ & $7(83)$ & $950(82)$ & $1.5(84)$ & $1.4 \mathrm{e}+22$ & $0.159(85)$ & $3.1 \pm 0.5(86)$ & M (87) & $\ldots$ \\
\hline V2468 Cyg & $7.4(88)$ & $2454534.2(88)$ & 10: & $1000(88)$ & $0.77(89)$ & $1.0 \mathrm{e}+22$ & $0.242(90)$ & $\ldots$ & $\mathrm{N}$ & $\ldots$ \\
\hline V2491 Cyg & $7.54(91)$ & $2454567.86(91)$ & $4.6(92)$ & $4860(93)$ & $0.43(94)$ & $4.7 e+21$ & 0.09580: (95) & $10.5(96)$ & $\mathrm{N}$ & M \\
\hline V2487 Oph & $9.5(97)$ & $2450979.5(97)$ & $6.3(98)$ & $10000(98)$ & $0.38 \pm 0.08(98)$ & $2.0 \mathrm{e}+21$ & $\ldots$ & $27.5 \pm 3(99)$ & N (100) & Y (101) \\
\hline V2540 Oph & $8.5(102)$ & $2452295.5(102)$ & $\ldots$ & $\ldots$ & $\ldots$ & $2.3 e+21$ & $0.284781(103)$ & $5.2 \pm 0.8(103)$ & $\mathrm{N}$ & $\ldots$ \\
\hline V2575 Oph & $11.1(104)$ & $2453778.8(104)$ & 20: & $560(104)$ & $1.4(105)$ & $3.3 e+21$ & $\ldots$ & $\ldots$ & N (105) & $\ldots$ \\
\hline V2576 Oph & $9.2(106)$ & $2453832.5(106)$ & 8: & $1470(106)$ & $0.25(107)$ & $2.6 e+21$ & $\ldots$ & $\ldots$ & $\mathrm{N}$ & $\ldots$ \\
\hline V2615 Oph & $8.52(108)$ & $2454187.5(108)$ & $26.5(108)$ & 800 (109) & $0.9(108)$ & $3.1 \mathrm{e}+21$ & $\ldots$ & $3.7 \pm 0.2(108)$ & Y (110) & $\ldots$ \\
\hline V2670 Oph & $9.9(111)$ & $2454613.11(111)$ & 15: & $600(112)$ & 1.3: (113) & $2.9 e+21$ & . & $\cdot$ & N (114) & $\cdots$ \\
\hline V2671 Oph & $11.1(115)$ & $2454617.5(115)$ & 8: & $1210(116)$ & $2.0(117)$ & $3.3 e+21$ & $\ldots$ & $\ldots$ & M (117) & $\ldots$ \\
\hline V2672 Oph & $10.0(118)$ & $2455060.02(118)$ & $2.3(119)$ & $8000(118)$ & $1.6 \pm 0.1(119)$ & $4.0 \mathrm{e}+21$ & $\ldots$ & $19 \pm 2(119)$ & $\ldots$ & M \\
\hline V351 Pup & $6.5(120)$ & $2448617.5(120)$ & $16(121)$ & $\ldots$ & $0.72 \pm 0.1(122)$ & $6.2 e+21$ & $0.1182(123)$ & $2.7 \pm 0.7(122)$ & $\mathrm{N}$ & $\ldots$ \\
\hline V382 Nor & $8.9(124)$ & $2453447.5(124)$ & $12(40)$ & $1850(23)$ & $\cdots$ & $1.7 e+22$ & $\ldots$ & $\ldots$ & $\ldots$ & $\cdots$ \\
\hline V382 Vel & $2.85(125)$ & $2451320.5(125)$ & $4.5(126)$ & $2400(126)$ & $0.05:(126)$ & $3.4 e+21$ & $0.146126(127)$ & $1.68 \pm 0.3(126)$ & $\mathrm{N}$ & $\ldots$ \\
\hline V407 Cyg & $6.8(128)$ & $2455266.314(128)$ & $5.9(129)$ & $2760(129)$ & $0.5 \pm 0.05$ & $8.8 e+21$ & $15595(131)$ & $2.7(131)$ & $\ldots$ & $\mathrm{Y}$ \\
\hline V458 Vul & $8.24(132)$ & 2454322.39 (132) & $7(133)$ & $1750(134)$ & $0.6(135)$ & $3.6 e+21$ & 0.06812255 (136) & $8.5 \pm 1.8(133)$ & N (135) & $\ldots$ \\
\hline V459 Vul & $7.57(137)$ & $2454461.5(137)$ & $18(138)$ & 910 (139) & $1.0(140)$ & $5.5 e+21$ & $\ldots$ & $3.65 \pm 1.35(138)$ & Y (140) & $\ldots$ \\
\hline V4633 Sgr & $7.8(141)$ & $2450895.5(141)$ & $19 \pm 3(142)$ & $1700(143)$ & $0.21(142)$ & $1.4 \mathrm{e}+21$ & $0.125576(144)$ & $8.9 \pm 2.5(142)$ & $\mathrm{N}$ & $\ldots$ \\
\hline V4643 Sgr & $8.07(145)$ & $2451965.867(145)$ & $4.8(146)$ & $4700(147)$ & $1.67(148)$ & $1.4 \mathrm{e}+22$ & $\ldots$ & $3(148)$ & $\mathrm{N}$ & $\ldots$ \\
\hline V4743 Sgr & $5.0(149)$ & 2452537.5 (149) & $9(150)$ & $2400(149)$ & $0.25(151)$ & $1.2 \mathrm{e}+21$ & $0.281(152)$ & $3.9 \pm 0.3(151)$ & $\mathrm{N}$ & $\ldots$ \\
\hline V4745 Sgr & $7.41(153)$ & $2452747.5(153)$ & $8.6(154)$ & $1600(155)$ & $0.1(154)$ & $9.0 \mathrm{e}+20$ & $0.20782(156)$ & $14 \pm 5(154)$ & $\ldots$ & $\ldots$ \\
\hline V476 Sct & $10.3(157)$ & 2453643.5 (157) & $15(158)$ & $\ldots$ & $1.9(158)$ & $1.2 \mathrm{e}+22$ & $\ldots$ & $4 \pm 1(158)$ & M (159) & $\ldots$ \\
\hline V477 Sct & $9.8(160)$ & $2453655.5(160)$ & $3(160)$ & $2900(161)$ & 1.2: (162) & $4 e+21$ & $\ldots$ & $\ldots$ & M (163) & $\ldots$ \\
\hline V5114 Sgr & $8.38(164)$ & $2453081.5(164)$ & $11(165)$ & $2000(23)$ & $\ldots$ & $1.5 e+21$ & $\ldots$ & $7.7 \pm 0.7(165)$ & N (166) & $\ldots$ \\
\hline V5115 Sgr & 7.7 (167) & $2453459.5(167)$ & $7(40)$ & $1300(168)$ & 0.53 (169) & $2.3 e+21$ & $\ldots$ & $\ldots$ & N (169) & $\ldots$ \\
\hline V5116 Sgr & $8.15(170)$ & $2453556.91(170)$ & $6.5(171)$ & 970 (172) & $0.25(173)$ & $1.5 e+21$ & $0.1238(171)$ & $11 \pm 3(173)$ & $\mathrm{N}(174)$ & $\ldots$ \\
\hline V5558 Sgr & $6.53(175)$ & $2454291.5(175)$ & 125 (176) & 1000 (177) & $0.80(178)$ & $1.6 e+22$ & $\ldots$ & $1.3 \pm 0.3(176)$ & N (179) & $\ldots$ \\
\hline V5579 Sgr & $5.56(180)$ & $2454579.62(180)$ & 7: & $1500(23)$ & $1.2(181)$ & $3.3 e+21$ & $\ldots$ & $\ldots$ & Y (181) & \\
\hline V5583 Sgr & $7.43(182)$ & 2455051.07 (182) & 5: & $2300(182)$ & $0.39(30)$ & $2.0 \mathrm{e}+21$ & $\ldots$ & 10.5 & $\ldots$ & $\ldots$ \\
\hline V574 Pup & $6.93(183)$ & $2453332.22(183)$ & $13(184)$ & $2800(184)$ & $0.5 \pm 0.1$ & $6.2 \mathrm{e}+21$ & $\ldots$ & $6.5 \pm 1$ & M (185) & $\ldots$ \\
\hline V597 Pup & $7.0(186)$ & 2454418.75 (186) & 3: & $1800(187)$ & $0.3(188)$ & $5.0 \mathrm{e}+21$ & 0.11119 (189) & $\ldots$ & N (188) & $\ldots$ \\
\hline V598 Pup & $3.46(14)$ & 2454257.79 (14) & $9 \pm 1(190)$ & $\ldots$ & $0.16(190)$ & $1.4 \mathrm{e}+21$ & $\ldots$ & $2.95 \pm 0.8(190)$ & $\ldots$ & $\ldots$ \\
\hline V679 Car & $7.55(191)$ & 2454797.77 (191) & 20: & $\ldots$ & $\ldots$ & $1.3 e+22$ & $\ldots$ & $\ldots$ & $\ldots$ & $\ldots$ \\
\hline V723 Cas & $7.1(192)$ & $2450069.0(192)$ & $263(2)$ & $600(193)$ & 0.5 (194) & $2.35 \mathrm{e}+21$ & $0.69(195)$ & $3.86 \pm 0.23(196)$ & $\mathrm{N}$ & $\ldots$ \\
\hline V838 Her & 5 (197) & 2448340.5 (197) & $2(198)$ & $\ldots$ & $0.5 \pm 0.1$ & $2.6 e+21$ & 0.2975 (199) & $3 \pm 1(198)$ & Y (200) & $\ldots$ \\
\hline XMMSL1 J06 ${ }^{\mathrm{j}}$ & $12(201)$ & $2453643.5(202)$ & $8 \pm 2(202)$ & $\ldots$ & $0.15(203)$ & $8.7 e+20$ & $\ldots$ & 50 & $\ldots$ & $\ldots$ \\
\hline
\end{tabular}


Notes. Numbers in parenthesis are the reference codes

a Novae with Swift observations are presented in bold.

b Visual maximum.

c Date of visual maximum.

d As measured from the visual light curve. A ":" indicates an uncertain value due to an estimate from the AAVSO light curve.

e Of Balmer lines measured at or near visual maximum.

${ }^{\mathrm{f}}$ Average Galactic $N_{\mathrm{H}}$ within 0.5 of the nova position as given in the HEASARC $N_{\mathrm{H}}$ tool.

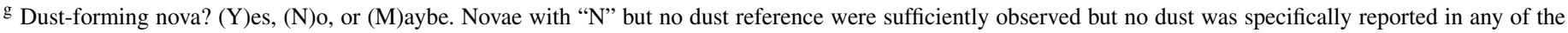
references.

${ }^{\mathrm{h}}$ Full nova name is CSS081007030559+054715.

${ }^{\mathrm{i}}$ An averaged date based on available photometry.

j Full nova name is XMMSL1 J060636.2-694933.

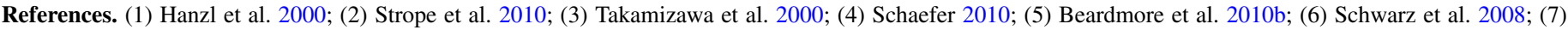

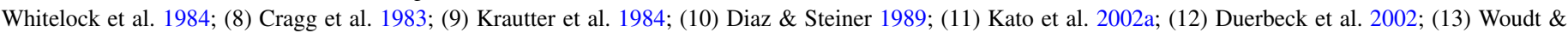

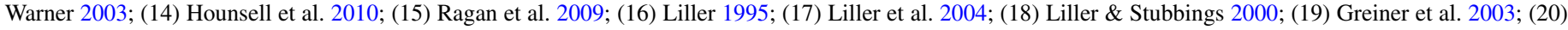

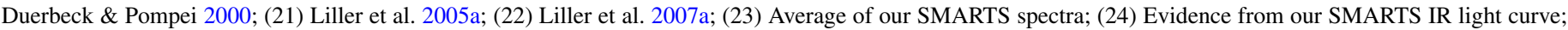

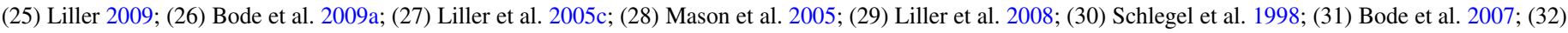

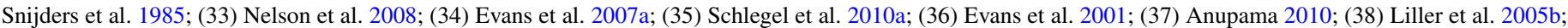

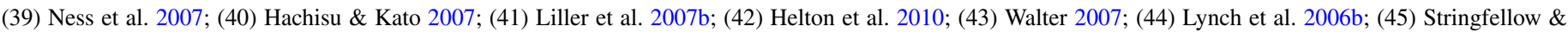

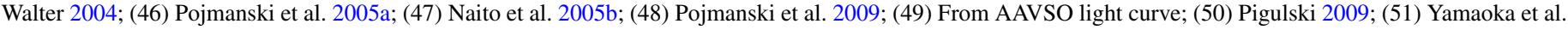

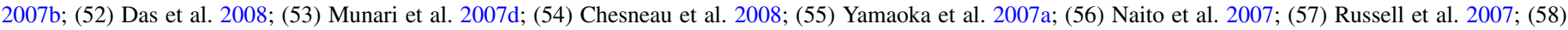

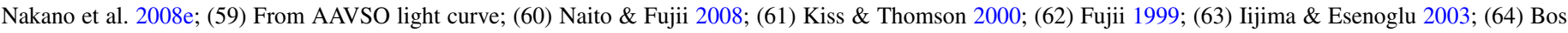

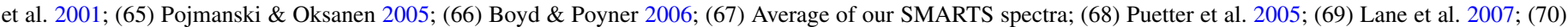

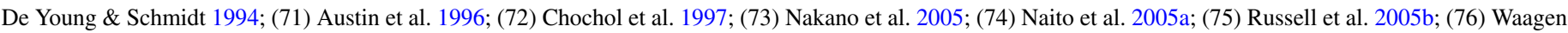

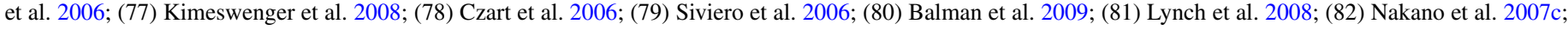

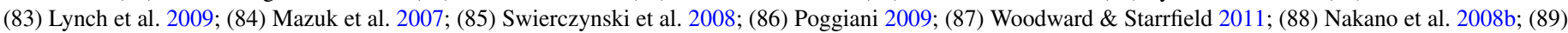

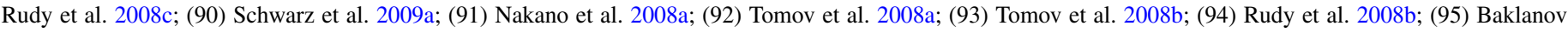

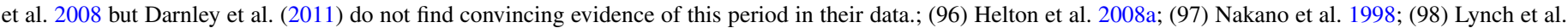

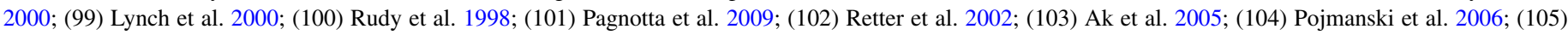

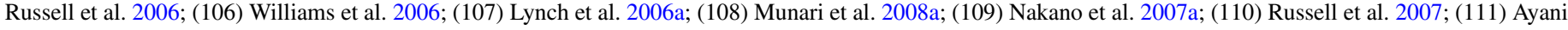

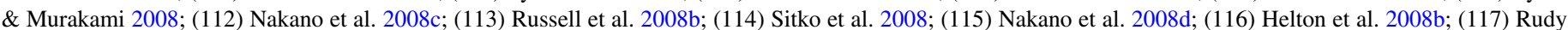

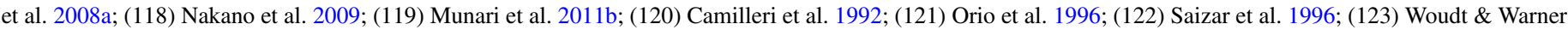

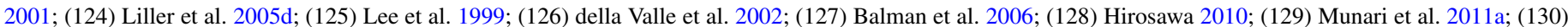

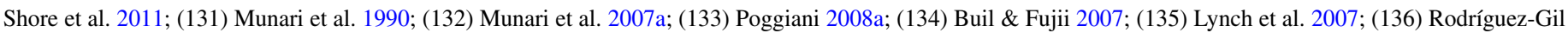

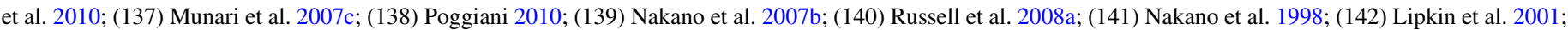

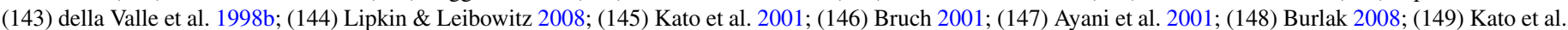

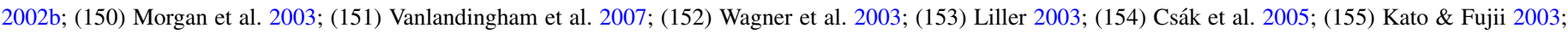

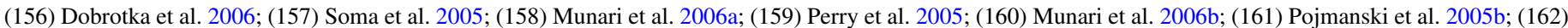

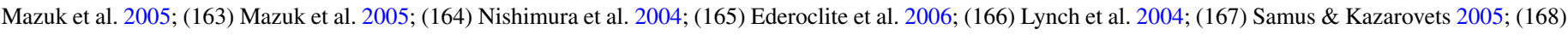

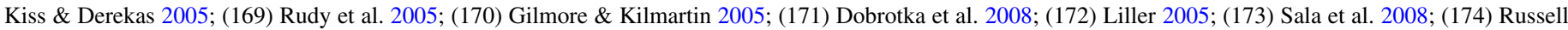

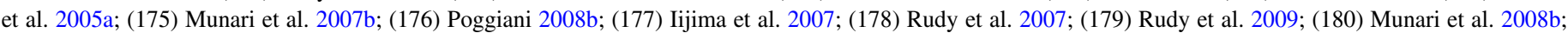

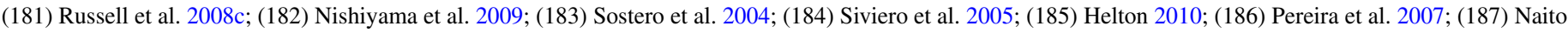

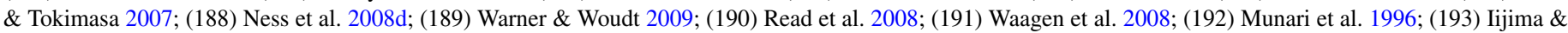

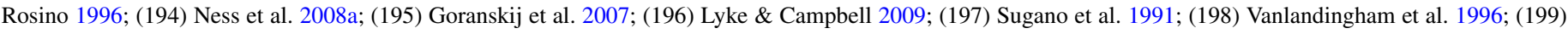
Szkody \& Ingram 1994; (200) Woodward et al. 1992; (201) Read et al. 2009; (202) Read et al. 2009; (203) Standard LMC value.

also presented. The last section, Section 6, provides a summary of this work.

\section{THE X-RAY DATA SET}

\subsection{Characteristics}

Table 1 presents the primary characteristics of all the Galactic/Magellanic Cloud novae with pointed X-ray observations prior to 2010 July 31 . In addition to the Swift data, the sample includes all the publicly available pointed observations from the ROSAT, XMM, Chandra, BeppoSAX, RXTE, and $A S C A$ archives. The columns give the nova name, visual magnitude at maximum, Julian date of visual maximum, time to decline 2 mag from visual maximum, the full width at halfmaximum (FWHM) of $\mathrm{H} \alpha$ or $\mathrm{H} \beta$ taken near visual maximum, $E(B-V)$ and averaged Galactic hydrogen column density, $N_{\mathrm{H}}$, along the line of sight, proposed orbital period, estimated distance, whether the nova was observed to form dust, and if the nova is a known RN. The numbers in the parentheses are the literature references given in the table notes. The names of novae with Swift observations are shown in bold.

Although P-Cygni absorption profiles provide the best values for the early velocities of the ejecta, they are not nearly as well reported in the literature as FWHMs of Balmer lines near maximum. Since nearly every nova has an FWHM citation as part of the spectroscopic confirmation of the initial visual detection, they are used as the expansion velocity proxy. Expansion velocities provide another way to classify a nova since more massive WDs eject less mass and at a greater velocity than low-mass WDs. This characteristic can be preferable to $t_{2}$ since the rate of decline can be difficult to determine for novae with secondary maxima or dust formation, or for those that 


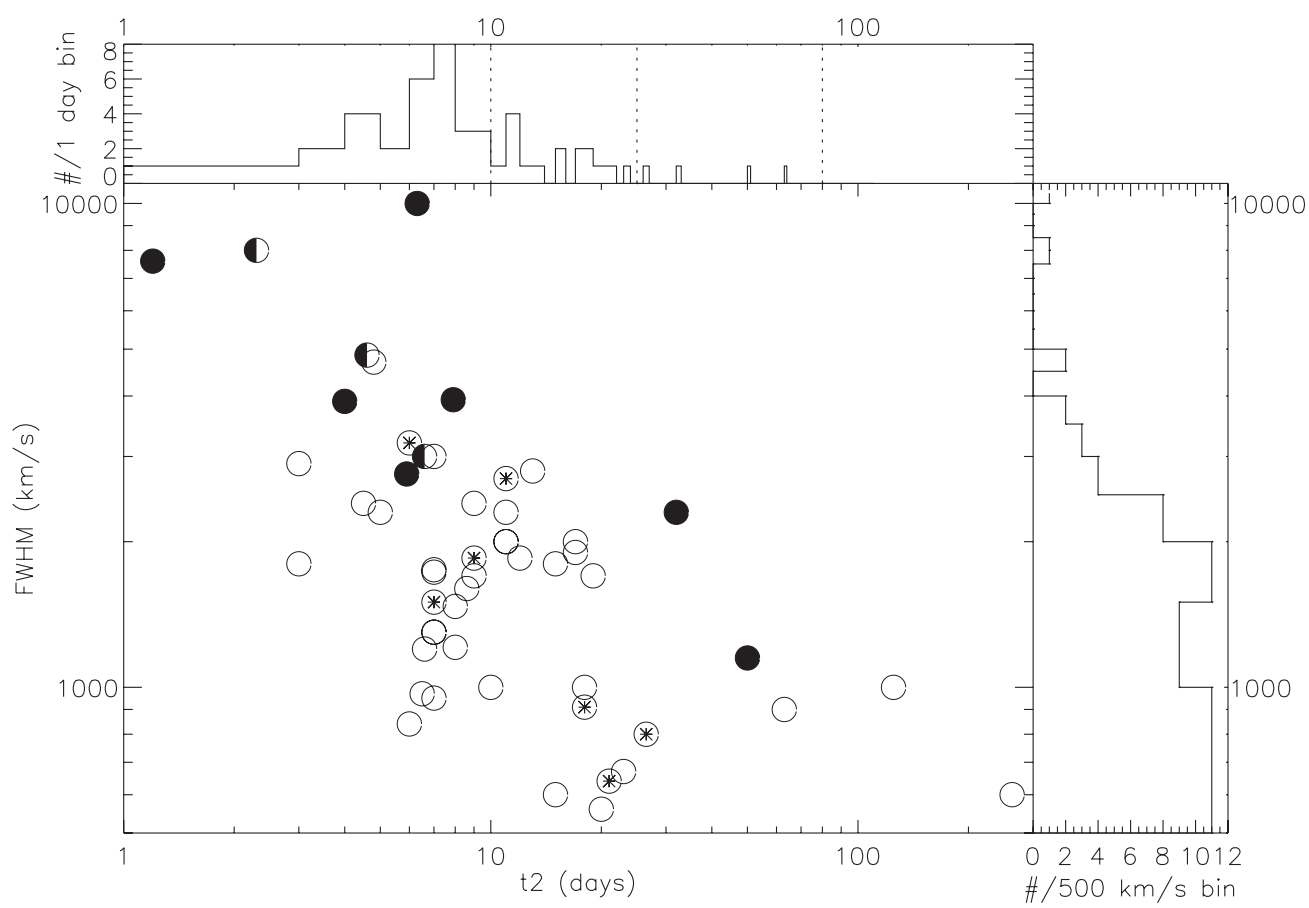

Figure 1. $t_{2}$ vs. FWHM near maximum for the novae in the sample. Filled circles are known RNe. Half filled circles are suspected RNe based on their characteristics. Circles with asterisks inside indicate dusty novae. The distribution histograms for $t_{2}$ and the FWHM are also shown in the secondary graphs. The dotted lines in the $t_{2}$ histogram show the boundaries between the "very fast," "fast," "moderately fast," and "slow" light curve classifications (Warner 2008). The majority of our novae belong to the "fast" or "very fast" classifications.

have poorly sampled early light curves. Both FWHM and $t_{2}$ are used as simple proxies for the WD mass. ${ }^{11}$ The $N_{\mathrm{H}}$ values were obtained from the HEASARC $N_{\mathrm{H}}$ tool $^{12}$ using the averaged LAB (Leiden/Argentine/Bonn Survey of Galactic H I Kalberla et al. 2005) and DL (Dickey \& Lockman 1990) maps within a 0.5 area around each nova.

Figure 1 shows the $t_{2}$ and FWHM distribution for all the novae with both values. The seven filled circles are known RNe, while the three half filled circles are suspected RNe. Dusty novae have an asterisk inside their circle symbols. As expected, the RNe tend toward large FWHM and fast $t_{2}$ times. In this sample the dusty novae are scattered throughout the FWHM- $t_{2}$ phase space showing no particular preference for any type of nova. Figure 1 also shows that there is a wide dispersion between FWHM and $t_{2}$, e.g., novae with $t_{2}$ of 10 days have FWHM values between 1000 and $3000 \mathrm{~km} \mathrm{~s}^{-1}$.

The top panel of Figure 1 shows the distribution of $t_{2}$ in one day bins. Using the light curve classifications of Warner (2008), the sample is heavily weighted toward very fast $\left(t_{2}<\right.$ 10 days) and fast (11 days $<t_{2}<25$ days) novae. These are intrinsically more luminous, with a larger rise from quiescence to maximum light. The peak is at eight days and with a median $t_{2}$ of nine days. There are only five novae in the entire sample with $t_{2}$ times greater than 50 days, IM Nor, LMC 2005, QY Mus, V723 Cas, and V5558 Sgr. The far right panel in Figure 1 gives the distribution for FWHM in $500 \mathrm{~km} \mathrm{~s}^{-1}$ bins. The majority of the novae in the sample have low expansion velocities with the peak in the $1500-2000 \mathrm{~km} \mathrm{~s}^{-1}$ bin. The median FWHM is $1800 \mathrm{~km} \mathrm{~s}^{-1}$. There are only five novae with FWHM $\geqslant$

\footnotetext{
11 While many other parameters also affect these observables, such as the accretion rate, these parameters are generally not known for specific novae and thus their contributions to the secular evolution cannot be determined.

12 http://heasarc.gsfc.nasa.gov/cgi-bin/Tools/w3nh/w3nh.pl
}

$4000 \mathrm{~km} \mathrm{~s}^{-1}$ in the sample and all but V4643 Sgr are RNe or suspected RNe.

The X-ray sample is biased toward fast novae for multiple reasons. The bulk of the observations are from Swift, and Swift has only been operational for five years. Fast systems, such as the RN RS Oph, will rise and fall on timescales of months (see Section 5.3), whereas slow novae, such as V1280 Sco, have not yet had sufficient time to evolve into soft X-ray sources (and may not) thus are therefore underrepresented. Slow novae also require more observing time to be monitored over their lifetime, particularly if the same coverage of the X-ray evolution is desired. Allocations of Swift observing time over multiple cycles are difficult to justify and execute unless a compelling scientific rationale is forthcoming, such as unusual or significant spectral variations (see Section 5.6), count rate oscillations, abundance pattern changes, etc. Slow and old novae (many tens of months post-outburst) are generally sampled once a year in part due to their slow evolution. However, the main reason the sample depicted in Figure 1 favors fast novae is due to the strong selection effect toward outbursts on high-mass WDs. While high-mass WDs, e.g., $\geqslant 1.2 M_{\odot}$, are relatively rare in the field, the timescale between outbursts is significantly shorter than for low-mass WDs, meaning they dominate any observational sample (Livio \& Truran 1994). Finally, high-mass WDs give rise to more luminous outbursts and the Swift Nova-CV group has a $V<8$ mag selection criterion, which leads to preferentially selecting brighter sources.

Figure 2 shows the distribution of our sample (Table 1) with respect to dust formation frequency. Only $\sim 16 \%$ of the novae in the sample had clear indications in the literature of dust formation in the ejecta. The dust formation frequency increases to $31 \%$ when including the seven novae where dust likely formed based on characteristics of the visual light curve but not yet confirmed by a measured SED excess in the thermal- and mid- 


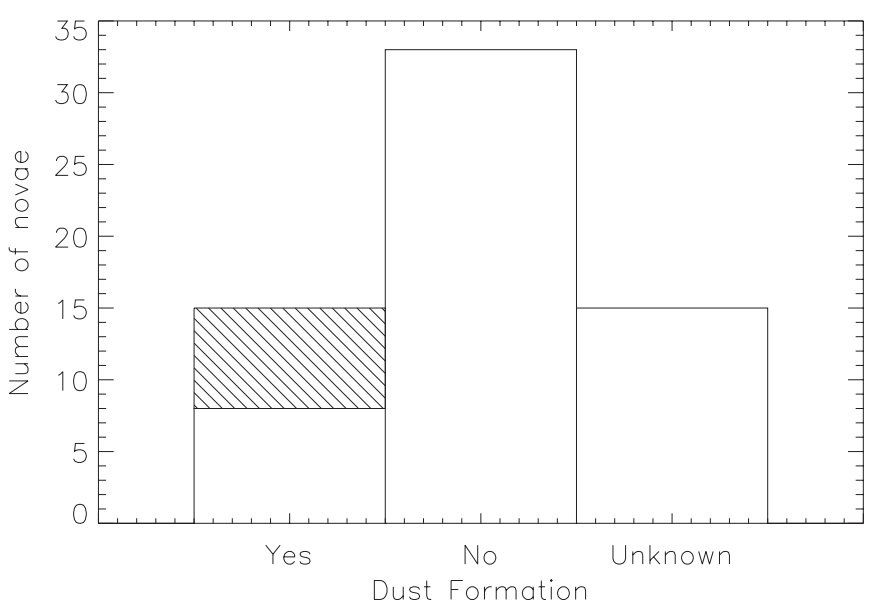

Figure 2. Distribution of dusty novae in the sample. The cross-hatched region is for novae that showed strong dust characteristics; however, the presence of dust in these systems has not been spectrophotometrically corroborated at IR wavelengths. The majority of novae in our X-ray selected sample (Table 1) did not form dust.

infrared, e.g., the "maybe"s in Column 10 of Table 1. This is consistent with the expectations of the general population of dusty novae which ranges from $18 \%$ to $\sim 40 \%$. The lower limit is set by Strope et al. (2010), who find that 93 well-sampled American Association of Variable Star Observers (AAVSO) novae have the large dip in their visual light curves indicative of strong dust formation (see Gehrz et al. 1998). The upper limit is from a recent Spitzer survey of IR bright novae that finds many novae have weak dust emission signatures with little or no dip in the visual light curve especially at late epochs (many hundreds of days post-outburst) when emission from the dust envelope is a few $\mu$ Jy (Woodward \& Starrfield 2011; Helton 2010).

In order to obtain the best X-ray and UV data, it is desirable to target novae with low extinction along the line of sight. However, determining the extinction early in the outburst is challenging. $N_{\mathrm{H}}$ maps are crude since they sample large regions of the sky. The region size used to derive the $N_{\mathrm{H}}$ values in Table 1 was 0.5 . Typically just a handful of sight lines are available in regions of this relatively small size. The problem is exacerbated in inhomogeneous areas such as the Galactic plane, where most novae are found. The extinction maps of Schlegel et al. (1998) can be used to obtain $E(B-V)$ since their spatial resolution is significantly higher. However, the Schlegel et al. (1998) maps suffer from large errors in the Galactic plane, $|b|<5^{\circ}$. Maps also give the total Galactic line of sight with no information versus distance and thus provide only an upper limit. $E(B-V)$ can also be determined from indirect methods but these require either high resolution spectroscopy to measure ISM absorption lines (e.g., Na I D $\lambda 5890 \AA$ A; Munari \& Zwitter 1997), the line strengths of optical and near-IR spectroscopy of O I lines (Rudy et al. 1989), or extensive $B$ and $V$ photometry during the early outburst (e.g., intrinsic $(B-V)$ at $V_{\max }$ or $t_{2}$; van den Bergh \& Younger 1987). Finally, $E(B-V)$ estimates can be affected by other factors occurring during the outburst such as dust formation or intrinsic absorption from the ejecta while the expanding material is still dense.

It is therefore desirable to check that the general relationship between $N_{\mathrm{H}}$ and $E(B-V)$ holds for novae. Figure 3 shows $N_{\mathrm{H}}$ versus $E(B-V)$ for the novae in this paper with the dotted line showing the average Milky Way extinction law, $E(B-V)=N_{\mathrm{H}} / 4.8 \times 10^{21}$ (Bohlin et al. 1978), as the dotted line. The circles represent novae with Galactic latitudes,

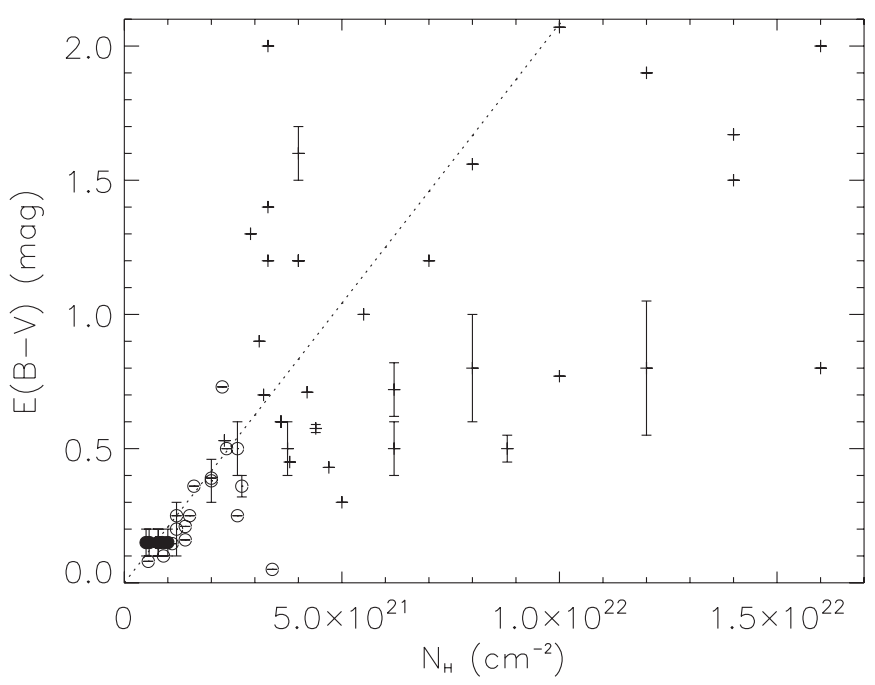

Figure 3. Local $N_{\mathrm{H}}$ value vs. the estimated $E(B-V)$. The values are from Table 1. The dotted line shows the $E(B-V)$ vs. $N_{\mathrm{H}}$ relationship of Bohlin et al. (1978). Circles are $|b| \geqslant 5^{\circ}$ novae, the $|b|<5^{\circ}$ novae are shown as pluses, and filled circles are Magellanic novae. Errors bars are given when available.

$|b| \geqslant 5^{\circ}$ while pluses are novae found within the disk, $|b|<$ $5^{\circ}$. Filled circles are Magellanic novae. Errors are present on all sources when available in the literature. There is good agreement with the relationship for novae with $E(B-V) \leqslant$ 0.6 and $N_{\mathrm{H}} \leqslant 2.9 \times 10^{21}$ with a correlation coefficient of 0.85 . These are primarily novae found outside of the galactic disk and thus fit the relationship well. Novae with these lowextinction values and column densities are ideal for soft X-ray detection. The relationship breaks down at larger values with a lower correlation coefficient of 0.64 for the entire sample as it is dominated by novae embedded within the Galactic disk. Novae with $E(B-V)$ values greater than 1.5 generally make poor SSS candidates due to the large extinction.

The maximum magnitude versus rate of decline relationship of della Valle \& Livio (1995) provides an estimate of the distances for the Galactic novae in Table 1. The distance estimate range extends from the relatively nearby V1280 Sco $(\sim 1 \mathrm{kpc})$ to the other side of the Galaxy for V2576 Oph ( $\sim 28 \mathrm{kpc})$. The median Galactic distance from this relationship is $5.5 \mathrm{kpc}$ for this sample.

\subsection{X-Ray Evolution}

All the available Swift XRT and UVOT data of novae in the public archive up to 2010 July 31 are presented in Table 2. The data were primarily obtained from pointed observations but a few serendipitous observations are also included. The full data set is available in the electronic edition with only V1281 Sco shown as an example here. The columns provide the Swift observation identification, exposure time, day of the observation from visual maximum (see Table 1), XRT total (0.3-10 keV) count rate, the Hard (1-10 keV) to Soft $(0.3-1 \mathrm{keV})$ hardness ratio, HR1, the Soft and Hard band count rates, the (Hard-Soft)/ (Hard+Soft) hardness ratio, HR2, and the uvw2 $\left(\lambda_{c}=1928 \AA\right)$, uvm2 (2246 ̊), uvw1 (2600 ̊), $u(3465 \AA), b(4392 \AA)$, and $v(5468 \AA)$ UVOT filter magnitudes, if available. The UVOT magnitudes do not include the systematic photometric calibration errors from Poole et al. (2008, Table 6).

There is one row in the table per observation ID, however this is not a fixed unit of time; most observation IDs are less than 0.13 days duration and the median exposure time is $1.76 \mathrm{ks}$. For 
Table 2

Swift XRT/UVOT Data for Novae in the Archive

\begin{tabular}{|c|c|c|c|c|c|c|c|c|c|c|c|c|c|}
\hline ObsID & $\begin{array}{l}\text { Exp } \\
(\mathrm{ks})\end{array}$ & Day $^{\mathrm{a}}$ & 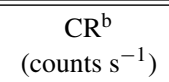 & $\mathrm{HR}^{\mathrm{c}}$ & $\begin{array}{c}\text { Soft } \\
\left(\text { counts s}^{-1}\right)\end{array}$ & $\begin{array}{c}\text { Hard } \\
\left(\text { counts s}^{-1}\right)\end{array}$ & $\mathrm{HR} 2^{\mathrm{c}}$ & $\begin{array}{l}\text { uvw2 } \\
\text { (mag) }\end{array}$ & $\begin{array}{l}\text { uvm2 } \\
\text { (mag) }\end{array}$ & $\begin{array}{l}\text { uvw1 } \\
\text { (mag) }\end{array}$ & $\begin{array}{c}u \\
(\mathrm{mag})\end{array}$ & $\begin{array}{c}b \\
(\mathrm{mag})\end{array}$ & $\begin{array}{c}v \\
(\mathrm{mag})\end{array}$ \\
\hline \multicolumn{14}{|c|}{ V1281 Sco } \\
\hline 00030891001 & 3.87 & 2.95 & $<0.0030$ & $\cdots$ & $\ldots$ & $\ldots$ & $\cdots$ & $\ldots$ & . & $\ldots$ & $\ldots$ & $\ldots$ & . \\
\hline 00037164001 & 5.24 & 338.66 & $0.1634_{-0.0079}^{+0.0079}$ & $0.0090 \pm 0.0074$ & 0.1619 & 0.0015 & -0.98 & $\ldots$ & $\ldots$ & $\ldots$ & $\ldots$ & $\ldots$ & . \\
\hline 00037164002 & 3.45 & 344.07 & $0.2429_{-0.0120}^{+0.0120}$ & $0.0062 \pm 0.0057$ & 0.2414 & 0.0015 & -0.99 & 19.50 & 19.64 & 18.20 & . & $\ldots$ & . \\
\hline 00037164003 & 4.24 & 351.05 & $0.6376_{-0.0282}^{+0.0282}$ & $0.0047 \pm 0.0053$ & 0.6346 & 0.0030 & -0.99 & 20.32 & $\ldots$ & $\ldots$ & $\ldots$ & $\ldots$ & . \\
\hline 00037164005 & 1.66 & 361.10 & $0.2727_{-0.0185}^{+0.0185}$ & $0.0012 \pm 0.0081$ & 0.2723 & 0.0003 & -1.00 & 20.43 & . & . & . & . &. \\
\hline 00037164006 & 2.89 & 366.41 & $0.2284_{-0.0129}^{+0.0129}$ & $0.0097 \pm 0.0089$ & 0.2262 & 0.0022 & -0.98 & 20.42 & $\ldots$ & $\ldots$ & $\ldots$ & $\ldots$ & $\ldots$ \\
\hline 00037164007 & 2.02 & 432.69 & $0.0853_{-0.0079}^{+0.0079}$ & $0.0002 \pm 0.0063$ & 0.0853 & 0.0000 & -1.00 & 20.22 & $\cdots$ & 19.11 & $\cdots$ & $\cdots$ & . \\
\hline 00090248001 & 4.68 & 819.99 & $<0.0013$ & $\ldots$ & $\ldots$ & $\ldots$ & $\ldots$ & 20.32 & $>20.56$ & 20.07 & $\ldots$ & $\ldots$ & 19.30 \\
\hline
\end{tabular}

Notes.

a Days after visual maximum, see Table 1.

${ }^{\mathrm{b}}$ Corrected for PSF losses and bad columns. The $3 \sigma$ upper limits are given when there is no detection better than $3 \sigma$.

${ }^{\mathrm{c}}$ Hardness ratios defined as HR1 $=H / S$ and HR2 $=(H-S) /(H+S)$ with Hard $(H)=1-10 \mathrm{keV}$ and Soft $(S)=0.3-1 \mathrm{keV}$.

(This table is available in its entirety in a machine-readable form in the online journal. A portion is shown here for guidance regarding its form and content.)

this exposure time, our $3 \sigma$ detection limit is 0.0037 counts $\mathrm{s}^{-1}$ (0.3-10 keV, corrected for typical point-spread function (PSF) coverage). This corresponds to an unabsorbed flux limit in the same band, assuming absorption by $N_{\mathrm{H}}=3 \times 10^{20}$ of $1.5 \times$ $10^{-13}$ and $2.0 \times 10^{-13} \mathrm{erg} \mathrm{cm}^{-2} \mathrm{~s}^{-1}$ for a $5 \mathrm{keV}$ optically thin thermal spectrum and a $50 \mathrm{eV}$ blackbody spectrum, respectively.

To create a self-consistent data set for Table 2 we used the software described by Evans et al. (2007b, 2009). This extracts source and background event lists from the data (using an annular source region where necessary to eliminate pile up), and then bins these data to form the light curve, applying corrections for pile up, bad pixels and the finite size of the source region as necessary. Since novae tend to be soft, we chose the energy bands for the hardness ratio to be $0.3-1 \mathrm{keV}$ and $1-10 \mathrm{keV}$. There is also evidence that, for very soft sources, pile up occurs at lower count rates than for hard sources; we thus set the threshold at which pile up is considered a risk to be $0.3(80)$ count s $^{-1}$ in PC (WT) mode (the defaults from Evans et al. 2007b are 0.6 and 150 , respectively).

We chose to group the data in one bin per Swift observation. In the current version of the online software (for this binning mode), background subtraction is only carried out using Gaussian statistics, and does not produce upper limits if this results in a non-detection. We thus took the "detailed" light curves produced by the web tools, which include the number of measured counts in each bin, the exposure time, and the correction factor (accounting for pile up, etc.). Following the approach of Evans et al. (2007b) for other binning methods, where any bins had fewer than 15 detected source counts, we used the Bayesian method of Kraft et al. (1991) to determine whether the source was detected at the $3 \sigma$ level. If this was not the case, a $3 \sigma$ upper limit was produced using this Bayesian method, otherwise a data point with standard $1 \sigma$ uncertainty was produced using the Kraft et al. (1991) approach.

The hardness ratios were always calculated using Gaussian statistics, unless one band had zero-detected source photons: in this case no ratio could be produced. The hardness ratios were defined as HR1 $=H / S$ and HR2 $=(H-S) /(H+S)$ where $H=$ $1.0-10 \mathrm{keV}$ and $S=0.3-1.0 \mathrm{keV}$.

Figures 4 and 5 show the X-ray observations of the Swift novae as a function of time since visual maximum. The novae are organized from bottom to top in increasing FWHM values (Table 1), with the FWHM alternating on the left and right sides of the figures. Novae with unknown FWHM are labeled "U" and placed at the bottom. Figure 4 shows the novae with confirmed SSS emission while Figure 5 shows the novae with no current SSS detections. Note that some novae in Figure 5, particularly the slowly evolving ones V5558 Sgr and V2468 Cyg, may eventually evolve to the SSS phase. Figure 6 is the same but for well-observed SSS novae observed prior to the launch of Swift; these novae typically have much poorer observational coverage. The black stars are the individual Swift observations. The figures also contain supplemental observations obtained with other X-ray facilities, Chandra, XMM, ASCA, RXTE, BeppoSAX, and ROSAT, which are shown as circles, downward pointing triangles, upward pointing triangles, yellow squares, diamonds, and red squares, respectively. The colors associated with each bar give the type of emission observed based on the hardness ratio. Red bars indicate time intervals when the HR2 of an individual source was $\lesssim-0.3$ and the uncertainty in the relative error was $<5 \%$; the photons in this case are primarily soft and these regions are associated with the SSS phase. Orange bars designate observations with the same hardness ratio but larger errors. Yellow shows regions between observations where hard/ soft change occurred. The orange and yellow regions represent the maximum limits of the soft phase since the transition occurred at some point during these times. Section 4 describes the SSS phase in greater detail. Green regions show times when the overall detected spectrum was hard, HR2 $>-0.3$ and Section 2.2 discusses this phase. Finally, blue represents times of non-detections. Table 3 also gives the color descriptions for Figures 4-6.

Several trends are evident in Figures 4-6. As the FWHM decreases, the novae in the sample become SSS later and remain in the SSS phase longer. This behavior is consistent with larger expansion velocity novae originating on higher mass WDs (Starrfield et al. 2009). In addition the early, hard detections are generally only observed in the high FWHM novae. The trends evident in Figures 4 and 6 allow for a straightforward interpretation of Figure 5-fast novae (loci at the top of the panel) are infrequently observed in the SSS phase as early $\mathrm{X}$-ray observations of these systems is often absent. The slower 


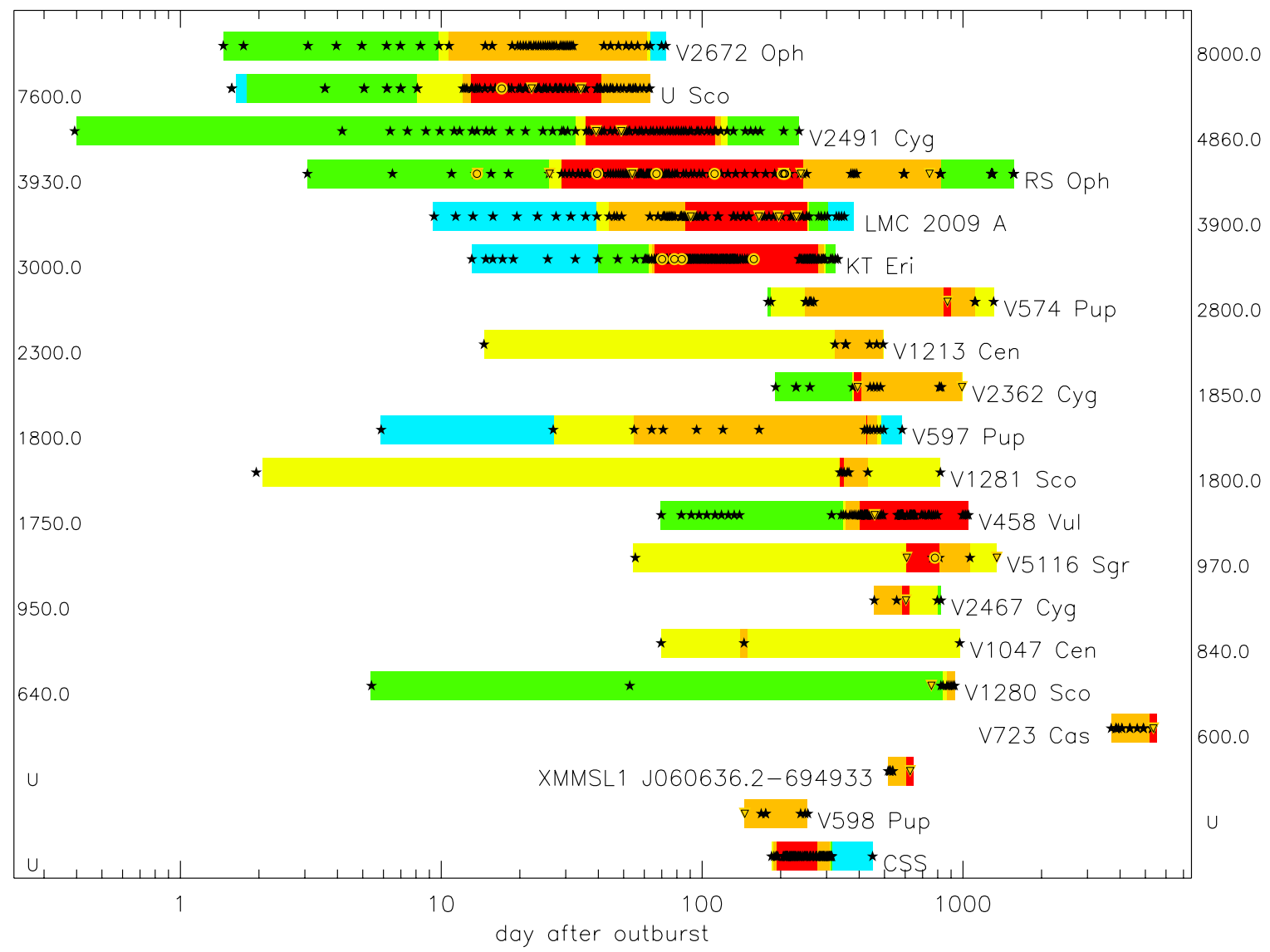

Figure 4. X-ray epochs for Swift sources with the best SSS phase coverage. The novae are arranged by increasing optical emission line FWHM with the FWHM values shown either left or right of the source. "U" is used for novae with unknown FWHM velocities. Refer to Table 3 for a summary of the color coding.

Table 3

Figures 4-6 Detection Definitions and Descriptions and Symbol Legend

\begin{tabular}{lll}
\hline \hline Color & \multicolumn{1}{c}{ HR2 ${ }^{\mathrm{a}}$ and Error } & \multicolumn{1}{c}{ X-Ray Emission } \\
\hline Blue & $\ldots$ & Undetected \\
Green & $>-0.3$ & Hard \\
Yellow & $\ldots$ & Transition between Green and Orange/Red classification. \\
Orange & $\lesssim-0.3 \%$ and $>5 \%$ error & Soft but with large uncertainty, highly variable during initial rise. \\
Red & $\lesssim-0.3 \%$ and $<5 \%$ error & Soft X-rays \\
\hline & & \\
\hline Swift & Stars & \\
Chandra & Circles & \\
XMM & Downward pointing triangles & \\
ASCA & Downward pointing triangles & \\
$R X T E$ & Yellow squares & \\
BeppoSAX & Diamonds & \\
ROSAT & Red squares & \\
\hline
\end{tabular}

Note. ${ }^{\text {a }} \mathrm{HR} 2=(H-S) /(H+S)$ with $\operatorname{Hard}(H)=1-10 \mathrm{keV}$ and Soft $(S)=0.3-1 \mathrm{keV}$.

novae at the bottom of the figure have not been followed with sufficient temporal coverage late in their evolution or they have not yet reached the SSS phase or have ceased nuclear burning before their ejecta clears sufficiently to observe SSS emission.

A note of caution about using Figures 4 and 5 to determine nuclear burning timescales is appropriate. These figures are based only on the strength and error of HR2 as provided in Table 2 which is based on a fixed hardness threshold for all novae in the table. Novae that have significant intrinsic hard emission such as V407 Cyg may not be classified as SSSs by this definition even though they have soft X-ray light curves typical of nuclear burning and cessation on the WD (see Section 4.3). High extinction will have a similar effect. The red regions generally also overestimate the duration of the SSS since that phase is also defined by a tremendous rise in the soft X-ray count rate. Sections 4.1 and 4.2 provide the determination of nuclear burning timescales for the X-ray nova sample.

For completeness, Table 4 gives a summary of all the publicly available, pointed XMM and Chandra nova observations. The columns are the nova name, the observational identifier, the exposure time, Julian date and day after visual maximum of the observation, and a short comment on the result of the 


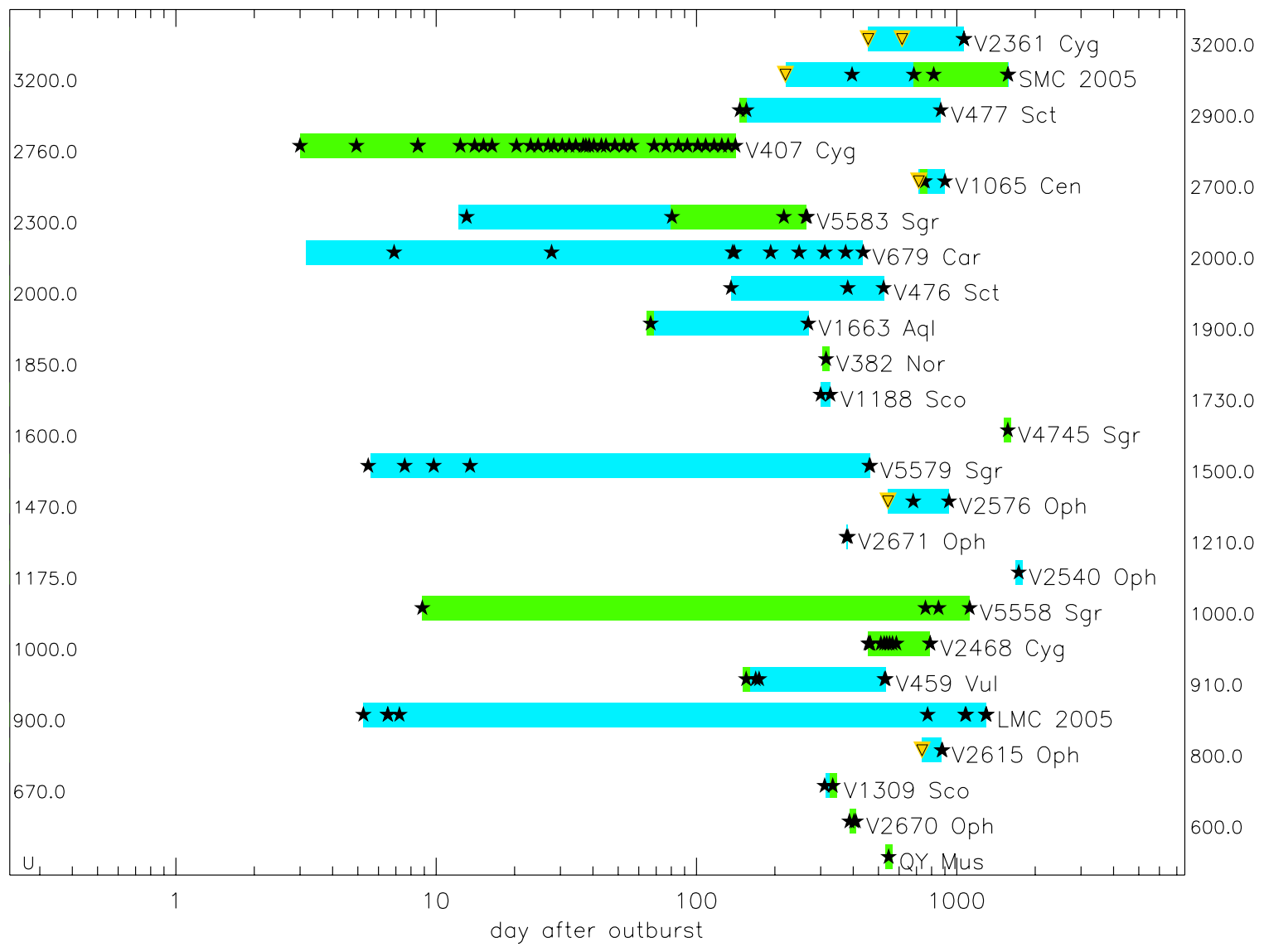

Figure 5. Same as Figure 4 except for the Swift sources without significant SSS detections.

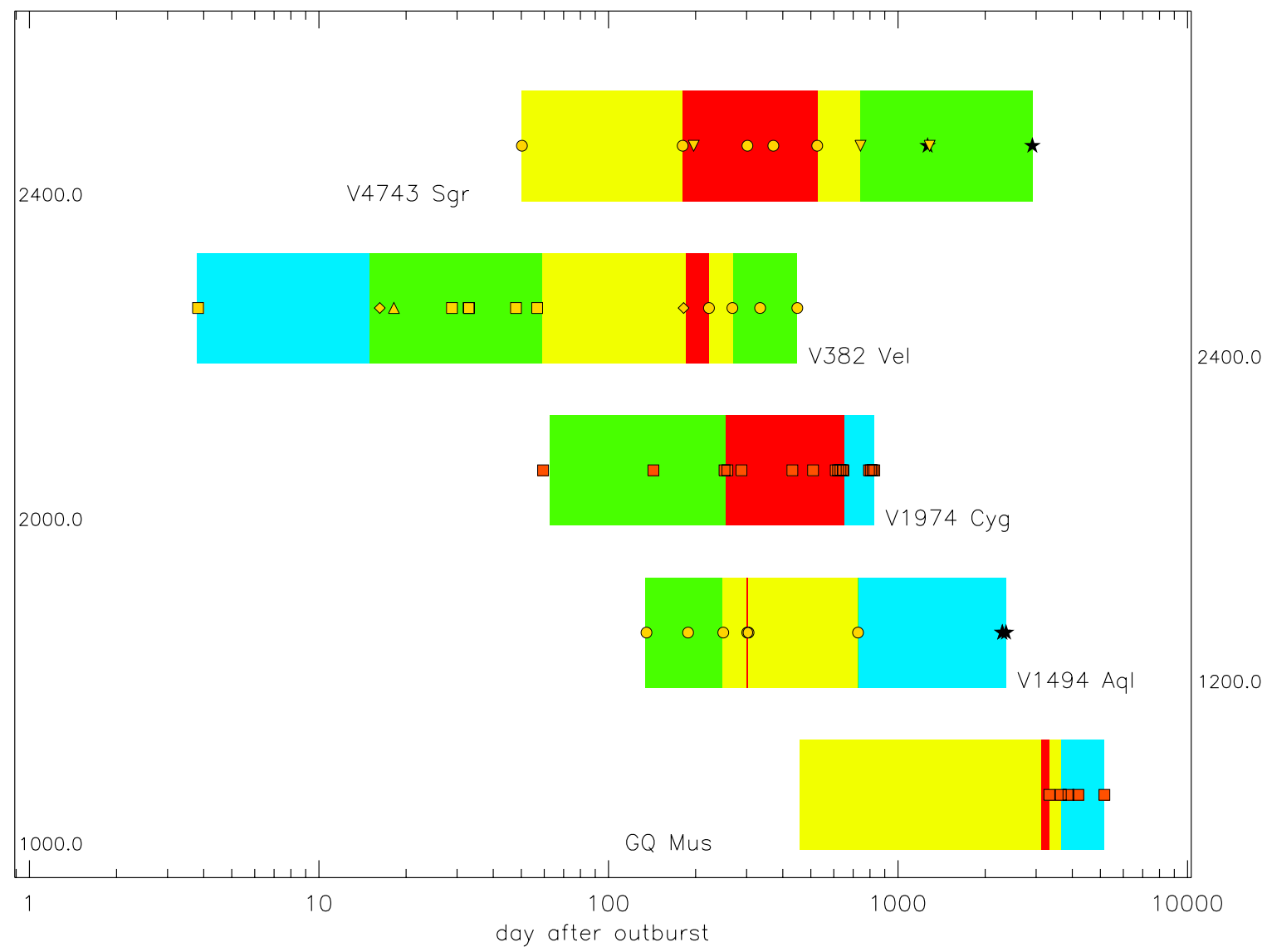

Figure 6. Same as Figure 4 but for pre-Swift SSS novae. 
Table 4

Pointed Chandra and XMM Observations of Recent Novae

\begin{tabular}{|c|c|c|c|c|c|}
\hline Name & ObsID $^{\mathrm{a}}$ & $\begin{array}{l}\text { Exp } \\
(\mathrm{ks})\end{array}$ & JD & Days $^{\mathrm{b}}$ & Result $^{\mathrm{c}}$ \\
\hline \multirow[t]{3}{*}{$\overline{\mathrm{CI}} \mathrm{Aql}$} & 2465 (ACIS-S) & 2.2 & 2452062 & 396 & Faint (1) \\
\hline & 2492 (ACIS-S) & 19.9 & 2452123 & 457 & Faint (1) \\
\hline & 0652760201 & 26.9 & 2455577 & 3912 & NPA \\
\hline $\operatorname{CSS} 081007^{\mathrm{d}}$ & 9970 (HRC-S/LETG) & 35.2 & 2454818 & 222: & SSS (2) \\
\hline \multirow[t]{2}{*}{ IM Nor } & 3434 (ACIS-S) & 5.6 & 2452317 & 28 & Not detected (3) \\
\hline & 2672 (ACIS-S) & 4.9 & 2452425 & 136 & Faint and hard (3) \\
\hline \multirow[t]{4}{*}{ KT Eri } & 12097 (HRC-S/LETG) & 15.2 & 2455219 & 69 & SSS (4) \\
\hline & 12100 (HRC-S/LETG) & 5.1 & 2455227 & 77 & SSS \\
\hline & 12101 (HRC-S/LETG) & 5.1 & 2455233 & 83 & SSS \\
\hline & 12203 (HRC-S/LETG) & 5.1 & 2455307 & 157 & SSS \\
\hline \multirow[t]{3}{*}{ Nova LMC 2000} & 0127720201 & 16.3 & 2451751 & 14 & Faint and hard (5) \\
\hline & 0127720301 & 10.0 & 2451785 & 48 & Hard (5) \\
\hline & 0127720401 & 10.5 & 2451998 & 291 & Not detected (5) \\
\hline \multirow[t]{4}{*}{ Nova LMC 2009a } & 0610000301 & 37.7 & 2454957 & 90 & SSS \\
\hline & 0610000501 & 58.1 & 2455032 & 165 & SSS \\
\hline & 0604590301 & 31.9 & 2455063 & 196 & SSS \\
\hline & 0604590401 & 51.2 & 2455097 & 230 & SSS \\
\hline Nova SMC 2005 & 0311590601 & 11.6 & 2453807 & 219 & Not detected \\
\hline \multirow[t]{3}{*}{ U Sco } & 12102 (HRC-S/LETG) & 23.2 & 2455241 & 17 & SSS (6) \\
\hline & 0650300201 & 63.8 & 2455247 & 23 & SSS (7) \\
\hline & 0561580301 & 62.8 & 2455259 & 35 & SSS (7) \\
\hline V1065 Cen & 0555690301 & 9.4 & 2454837 & 714 & Not detected \\
\hline \multirow[t]{5}{*}{ V1187 Sco } & 4532 (ACIS-S) & 5.2 & 2453305 & 96 & $<2 \mathrm{keV}+\mathrm{N}$ vil line \\
\hline & 4533 (HRC-S/LETG) & 26.1 & 2453401 & 181 & Not detected \\
\hline & 0404431101 & 4.7 & 2454161 & 941 & Not detected \\
\hline & 0404430301 & 9.4 & 2454161 & 941 & Not detected \\
\hline & 0555691001 & 7.1 & 2454904 & 1684 & Not detected \\
\hline V1280 Sco & 0555690601 & 4.5 & 2454903 & 756 & $1 \mathrm{keV}$ emission \\
\hline \multirow[t]{2}{*}{ V2361 Cyg } & 0405600101 & 11.1 & 2453868 & 456 & Not detected \\
\hline & 0405600401 & 14.9 & 2454028 & 616 & Not detected \\
\hline \multirow[t]{2}{*}{ V2362 Cyg } & 0506050101 & 9.9 & 2454225 & 394 & Thermal plasma (8) \\
\hline & 0550190501 & 27.9 & 2454821 & 990 & Very weak \\
\hline V2467 Cyg & 0555690501 & 7.0 & 2454780 & 605 & SSS \\
\hline \multirow[t]{4}{*}{ V2487 Oph } & 0085580401 & 8.3 & 2451965 & 986 & Thermal plasma (9) \\
\hline & 0085581401 & 8.1 & 2452157 & 1178 & Thermal plasma (9) \\
\hline & 0085581701 & 7.6 & 2452331 & 1352 & Thermal plasma (9) \\
\hline & 0085582001 & 8.5 & 2452541 & 1562 & Thermal plasma $+\mathrm{Fe} \mathrm{K} \alpha$ line (9) \\
\hline \multirow[t]{2}{*}{ V2491 Cyg } & 0552270501 & 39.3 & 2454606 & 39 & SSS (10) \\
\hline & 0552270601 & 30.0 & 2454616 & 49 & SSS (11) \\
\hline V2575 Oph & 0506050201 & 14.9 & 2454347 & 569 & Not detected \\
\hline V2576 Oph & 0506050301 & 11.5 & 2454376 & 544 & Not detected \\
\hline V2615 Oph & 0555690401 & 9.7 & 2454922 & 735 & Not detected \\
\hline V351 Pup & 0304010101 & 51.8 & 2453525 & 4908 & Faint \\
\hline V458 Vul & 0555691401 & 11.7 & 2454780 & 459 & Weak $1 \mathrm{keV}$ emission \\
\hline \multirow[t]{3}{*}{ V4633 Sgr } & 0085580301 & 10.2 & 2451828 & 933 & Weak (12) \\
\hline & 0085581201 & 7.3 & 2451977 & 1082 & Weak (12) \\
\hline & 0085581301 & 11.6 & 2452159 & 1264 & Weak (12) \\
\hline \multirow[t]{2}{*}{ V4643 Sgr } & 0148090101 & 11.9 & 2452716 & 750 & Not detected \\
\hline & 0148090501 & 11.0 & 2452894 & 928 & Not detected \\
\hline \multirow[t]{2}{*}{ V5114 Sgr } & 0404430401 & 7.9 & 2454167 & 1086 & Not detected \\
\hline & 0404431201 & 3.6 & 2454167 & 1086 & Not detected \\
\hline \multirow[t]{2}{*}{ V5115 Sgr } & 0405600301 & 9.2 & 2454005 & 566 & Weak SSS \\
\hline & 0550190201 & 14.9 & 2454925 & 1486 & Weak detection \\
\hline \multirow[t]{3}{*}{ V5116 Sgr } & 0405600201 & 12.9 & 2454164 & 608 & SSS (13) \\
\hline & 7462 (HRC-S/LETG) & 35.2 & 2454336 & 780 & SSS (14) \\
\hline & 0550190101 & 26.6 & 2454893 & 1337 & Not detected \\
\hline V574 Pup & 0404430201 & 16.6 & 2454203 & 872 & SSS \\
\hline V598 Pup & 0510010901 & 5.5 & 2454402 & 146 & SSS (15) \\
\hline XMMSL1 J060636 & 0510010501 & 8.9 & 2454270 & 627 & SSS (16) \\
\hline
\end{tabular}

Notes.

${ }^{a}$ Chandra observations have a four digit IDs and are followed by the instrument configuration. XMM observations have 10 digit IDs.

${ }^{\mathrm{b}}$ Days since visual maximum, see Table 1.

${ }^{\mathrm{c}}$ The number in parenthesis is the code to the published data. NPA stands for "Not Publicly Available" and indicates proprietary observations at the time of this publication.

${ }^{\mathrm{d}}$ Full novae names are CSS081007030559+054715 and XMMSL1 J060636.2-694933.

References. (1) Greiner \& Di Stefano 2002; (2) Nelson \& Orio 2009; (3) Orio et al. 2005; (4) Ness et al. 2010a; (5) Greiner et al. 2003; (6) Orio et al. 2010; (7) Ness et al. 2010b; (8) Hernanz et al. 2007; (9) Ferri et al. 2007; (10) Ness et al. 2008c; (11) Ness et al. 2008b; (12) Hernanz \& Sala 2007; (13) Sala et al. 2008; (14) Nelson \& Orio 2007; (15) Read et al. 2008; (16) Read et al. 2009. 
observation. The instrument setup is also given in the second column for the Chandra observations. In some cases this data set provides important information on the SSS status of some sources due to a lack of or weak Swift detections. An example would be the XMM observations of V574 Pup, which confirms that there was a strong SSS during the interval between 2005 and 2007 when there were no Swift data (Helton 2010).

\section{THE EARLY HARD X-RAY PHASE}

Some novae have hard X-ray emission, e.g., $>1 \mathrm{keV}$, early in the outburst. These novae tend to be fast or RNe. This initial hard emission is thought to arise from shock-heated gas inside the ejecta or from collisions with external material, e.g., the wind of the red giant secondary in RS Oph (Bode et al. 2006; Sokoloski et al. 2006; Vaytet et al. 2007; Drake et al. 2009). Early hard X-ray emission observed in the very fast nova V838 Her has been attributed to intra-ejecta shocks from a secularly increasing ejection velocity (Lloyd et al. 1992; O'Brien et al. 1994). Much later in the outburst when nuclear burning has ceased hard X-rays can again dominate. These hard X-rays come from line emission from the ejected shell and/or emission from the accretion disk (Krautter 2002), or in the case of RS Oph, the re-emergence of the declining shocked wind emission once the SSS emission has faded (Bode et al. 2008).

Every nova with an FWHM $\geqslant 3000 \mathrm{~km} \mathrm{~s}^{-1}$ and observations within 100 days after visual maximum in the Swift sample exhibited hard X-rays. This detection rate is partially due to the fact that many of these novae were high interest targets, e.g., very bright at visual maximum (KT Eri), extreme ejection velocity (V2672 Oph), RN (RS Oph, V407 Cyg, and U Sco), detected prior to outbursts as an X-ray source (V2491 Cyg), etc.; thus their early X-ray evolution was well documented. In addition, a higher cadence of observations during the early phases greatly increased the probability of discovery.

The evidence of initial hard X-ray emission for slow novae is sparse as few were well sampled early in their outbursts. Only V458 Vul (Ness et al. 2009a; Tsujimoto et al. 2009) had early observations that showed a hard component with a duration of hundreds of days from its first observation $\sim 70$ days after visual maximum. The lack of significant evidence of hard emission in the early outburst of slow novae is consistent with shocks, either within the ejecta or with a pre-outburst ambient medium, being the primary source of early hard X-ray emission in the faster novae. Slower novae have lower ejection speeds and thus should either have less or delayed shock emission (see Equation (3) in Bode et al. 2006). Hard X-rays were also detected late in the outburst of novae with extreme and multiple ejection events. The best example of this is V2362 Cyg which at the time of its unusually bright secondary maximum had already doubled the width of its emission lines and was detected as a hard X-ray source (Lynch et al. 2008). Similarly the slow nova V5558 Sgr was also a late hard source. Its early light curve was marked by numerous secondary maxima similar to V723 Cas (Poggiani 2008b).

Another interesting case is the slow nova V1280 Sco, which was detected multiple times between days 834 and 939 after outburst as an X-ray source. Ness et al. (2009b) found that the X-ray count rate was relatively low and the SED was best fit with multiple thermal plasma models consistent with line emission. They attributed the lines from shock heating of the ejecta, but this is difficult to reconcile with how rapidly shock emission declines. Hounsell et al. (2010) showed that V1280 Sco had two bright secondary peaks after maximum. Thus, it is possible that this nova experienced additional ejection events later in the outburst that contributed the necessary energy to power shocks. Contemporary optical spectra from our Small and Moderate Aperture Research Telescope System (SMARTS) nova monitoring program show that the photosphere of V1280 Sco remains optically thick with P-Cygni profiles still present more than four years after outburst. Alternatively, the line emission may be from circumstellar gas photoionized by the initial X-ray pulse of the explosion. Given the relative proximity of V1280 Sco, ranging from $0.63 \pm 0.10 \mathrm{kpc}$ (Hounsell et al. 2010) to $1.6 \mathrm{kpc}$ (Chesneau et al. 2008), any X-ray emission lines would be much brighter than most novae in our sample, which has a larger median distance of $5.5 \mathrm{kpc}$. Unfortunately, V1280 Sco was X-ray faint making it impossible to determine the source of its X-ray emission.

\section{THE SSS PHASE}

\subsection{Rise to X-Ray Maximum and the "Turn-on" Time}

The unprecedented temporal coverage of the early outburst in X-rays with Swift has fully revealed a new phenomenon during the rise to X-ray maximum. Prior to Swift, V1974 Cyg had the best sampled X-ray light curve (see Figure 1 in Krautter et al. 1996). The 18 ROSAT observations showed a slow and monotonic rise to maximum. This light curve evolution was expected as the obscuration from the ejecta clears and the effective temperature of the WD photosphere increases (MacDonald et al. 1985). However, Chandra observations of V1494 Aql (Drake et al. 2003) and V4743 Sgr (Ness et al. 2003) hinted that this transition was not as smooth as previously observed, with short-term "bursts," periodic oscillations, and sudden declines.

With daily and sometimes hourly Swift coverage, the rise to X-ray maximum is unequivocally highly chaotic with large changes in the count rate evident in all well-observed Swift novae to date. Figure 7 illustrates this phenomenon in KT Eri from the data available in Table 2. During the initial rise to X-ray maximum, it exhibited large oscillations. The numerous large declines are even more dramatic when the observational data sets are not grouped by observation ID number as in Table 2 but broken into small increments (F. M. Walter et al. 2011, in preparation). At 76 days after visual maximum the variability became much smaller and the count rate stabilized around $\sim 150$ counts $\mathrm{s}^{-1}$. In addition to KT Eri (Bode et al. 2010), RS Oph (Osborne et al. 2011), U Sco (Schlegel et al. 2010b), nova LMC 2009a (Bode et al. 2009b; M. F. Bode et al. 2011, in preparation), V2672 Oph (Schwarz et al. 2009b), V2491 Cyg (Page et al. 2010; Ness et al. 2011), and V458 Vul (Ness et al. 2009a) all showed this large amplitude variability. The first three novae are known RNe while the next two and KT Eri are suspected to be RNe based on their observational characteristics. The fact that the less energetic V458 Vul also exhibited this phenomenon indicates that it is not just associated with very fast or RNe. See Section 5.3 for further discussion of nova variability.

The emergence of the SSS, referred to as "turn-on" time or $t_{\text {on }}$ hereafter, provides information on the mass of the ejected shell. The turn-on times for the novae in this sample are given in Table 5 . The $t_{\text {on }}$ time is defined as the time after visual maximum when the HR2 $<-0.8$ and there is significant increase in the soft count rate. Similarly the "turn-off" time $\left(t_{\text {off }}\right)$ is defined as the time after $t_{\text {on }}$ when the hardness ratio becomes harder, HR2 $>-0.8$, and the soft count rate declines rapidly as nuclear 


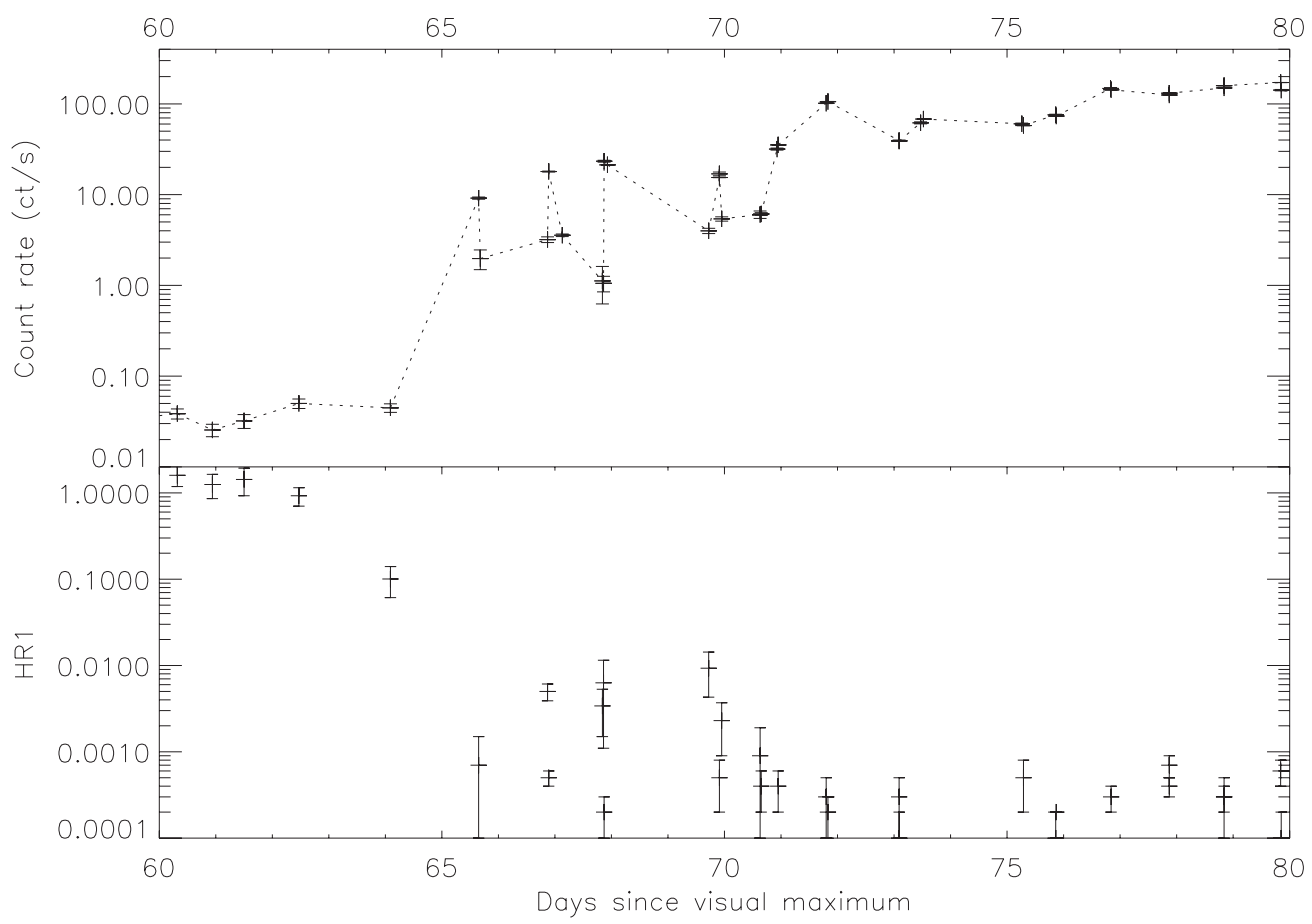

Figure 7. Early X-ray light curve of KT Eri in days since visual maximum. The top panel shows the count rate and the lower panel gives the hardness ratio, HR1. Dotted lines are added to the top panel to emphasize the variability. Prior to day 65 KT Eri was faint and hard. Between days 65 and 75 the source transitioned to the bright SSS phase with large amplitude oscillations in the count rate and some corresponding changes in HR1. After day 76 the both the count rate and hardness ratio significantly stabilized but still showed variability (see Section 5.3).

burning ends. Note that these definitions should not be confused with the SSS phases as shown in Figures $4-6$, as $t_{\text {on }}$ and $t_{\text {off }}$ also include the change in the soft count rate. SSS emission can only be observed when the ejecta column density declines to the point where the source can be observed. With the expansion velocity and turn-on time, upper limits on the ejected mass can be established. Shore (2008) gives the relationship (see Equation (9.2))

$$
M_{\text {eject }} \sim 6 \times 10^{-7} \phi N_{\mathrm{H}}(22) v_{\text {exp }}(1000)^{2} t_{\text {on }}^{2} M_{\odot},
$$

where $\phi$ is the filling factor, $N_{\mathrm{H}}(22)$ is the column density in units of $10^{22} \mathrm{~cm}^{-2}, v_{\exp }(1000)$ is the expansion velocity in units of $1000 \mathrm{~km} \mathrm{~s}^{-1}$, and $t_{\mathrm{on}}$ is the soft X-ray turn-on time in days and assumes spherical geometry. In this study, $\phi=0.1$ and a column density of $10^{22} \mathrm{~cm}^{-2}$ is used as the minimum $N_{\mathrm{H}}$ for the ejected shell to become transparent to soft X-rays. The expansion velocity is determined from $v_{\exp }=\mathrm{FWHM} / 2.355$ (Munari et al. 2010) where FWHM is the width of the Balmer lines near visual maximum as given in Table 1. Using the $t_{\mathrm{on}}$ times from Table 5, Figure 8 shows the estimated ejected masses as a function of ejection velocity. Note that the velocities derived from these FWHMs are lower limits as the X-ray opacity in the ejecta depends on faster material. This has the effect of shifting all the points in Figure 8 to the right. Accordingly the fastest novae, at the bottom right, U Sco and V2672 Oph, must have ejected much less than $10^{-5} M_{\odot}$ otherwise they would not have been observed as SSS sources so early after outburst. This inference is consistent with independent ejected mass estimates (e.g., U Sco; Anupama \& Dewangan 2000; Diaz et al. 2010; Drake \& Orlando 2010). Conversely, novae in the upper left corner must eject a significant amount of material. Large mass ejection events are also inferred from the optical spectra of novae like V1280 Sco which still showed P-Cygni lines three years after outburst (Sadakane et al. 2010) and a year later in our recent SMARTS spectroscopy.

Note that external extinction from the ISM is not taken into account in Figure 8 nor is the evolution of the effective temperature of the WD photosphere. Novae with large extinction may never be observed in the SSS phase, while a slow increase in the WD temperature after the ejecta has sufficiently cleared will delay the onset of $t_{\mathrm{on}}$, resulting in an overestimate of the ejected mass derived from Equation (1). Both factors along with deviations from the underlying assumptions such as different filling factors and non-spherical symmetry, can lead to different mass values given in Figure 8. These limitations explain why two novae with the same ejection velocities, V2468 Cyg and V5558 Sgr at $425 \mathrm{~km} \mathrm{~s}^{-1}$, can have divergent mass estimates due to different turn-on times.

\subsection{Turn-off Time}

Table 5 shows $t_{\text {off }}$ times or upper/lower limits for the novae in our sample. If optical light curve decline times, e.g., $t_{2}$, are used as simple proxies for WD masses then there should be a relationship between $t_{2}$ and duration of the SSS phase. In Figure 9 the turn-off time, $t_{\text {off }}$, is shown versus $t_{2}$. Overplotted as the solid line is the turn-off versus decline relationship of Hachisu \& Kato (2010, Equation (31)) where $t_{3}$ was converted to $t_{2}$ using Equation (7) in Hachisu \& Kato (2007). The combined uncertainties of both equations are represented by the two dotted lines. Hachisu \& Kato (2010) find that the time when nuclear burning ends is $\propto t_{\text {break }}^{1.5}$ (Equation (26)), where $t_{\text {break }}$ is the time of the steepening of their model free-free optical-IR light curves. This relationship is derived using a series of steady state models with a decreasing envelope mass to fit the observed multiwavelength light curves. The X-ray and UV light curves are fit with blackbodies while the optical and IR curves use optically thin, free-free emission. The parameters of the model 


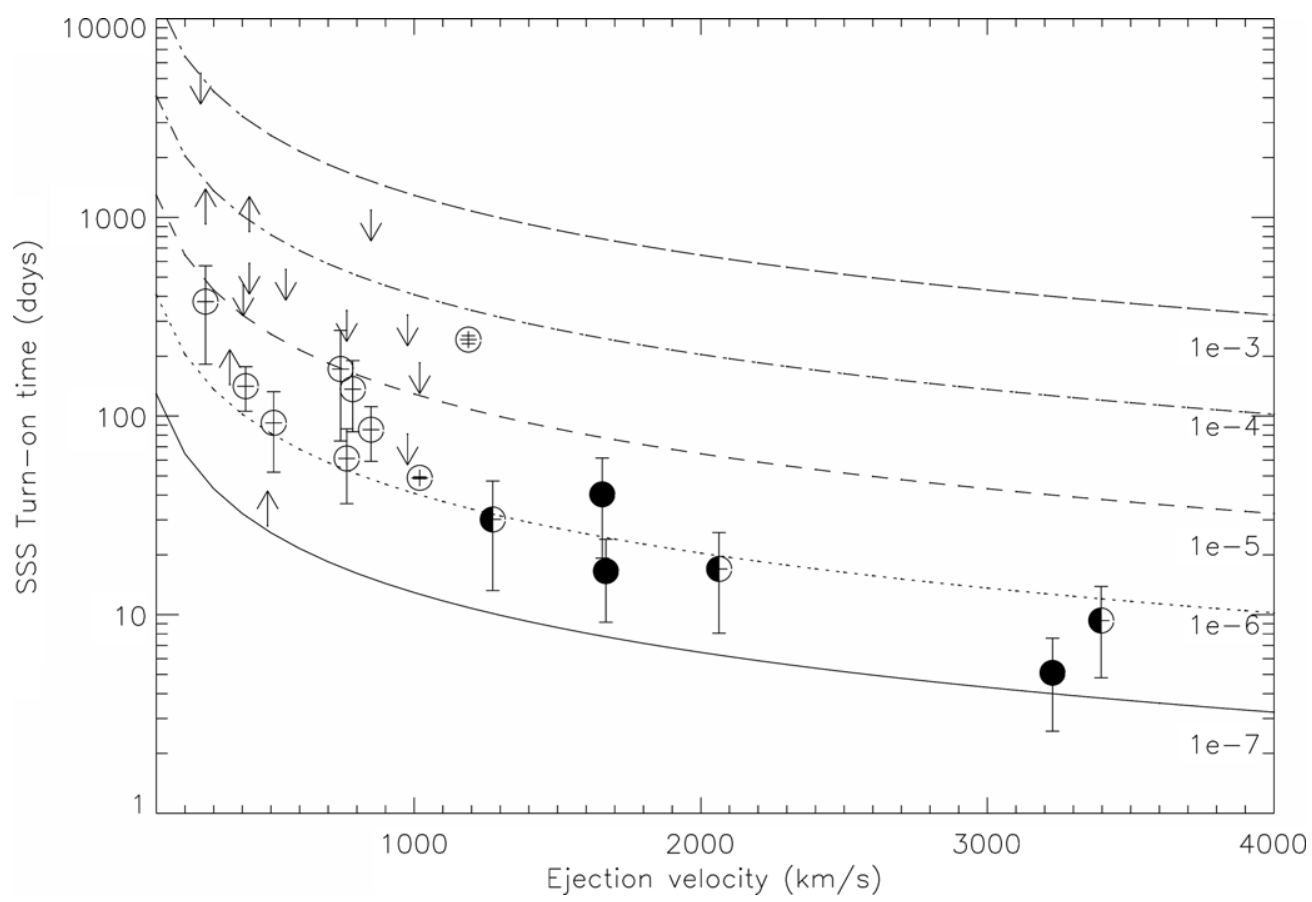

Figure 8. SSS turn-on time of novae (Table 5) as a function of the ejection velocity (estimated from the FWHMs in Table 1). Filled circles are known RNe. Half filled circles are suspected RNe based on their characteristics. Top to bottom: the lines show the relationship from Shore (2008, Equation (9.2)) for ejected masses of $1 \times$ $10^{-3}, 1 \times 10^{-4}, 1 \times 10^{-5}, 1 \times 10^{-6}$, and $1 \times 10^{-7} M_{\odot}$, respectively. The downward and upward arrows are estimated upper and lower limits.

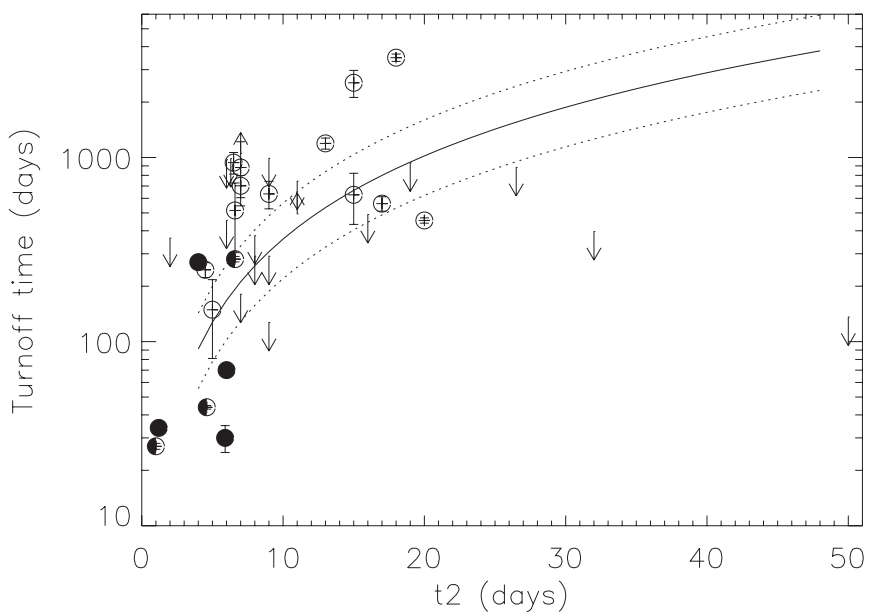

Figure 9. SSS turn-off time as a function of $t_{2}$ time with the Hachisu \& Kato (2010) relationship (solid line) and its associated uncertainty (dotted lines) overplotted. Upper and lower limits are also shown. Filled circles are known RNe. Half filled circles are suspected RNe based on their characteristics.

are the WD mass, composition of the WD envelope, and its mass prior to outburst. While the general trend is similar, the observed data do not fit the Hachisu \& Kato (2010) relationship, especially when the sample is expanded to include the novae with only upper or lower limits.

The relationship derived by Hachisu \& Kato (2010) utilizes the $t_{2}$ derived from the $y$-band light curve instead of the $V$ band as in this paper. The $y$ band is used by Hachisu \& Kato (2010) since it generally samples the continuum, whereas the $V$ band can have a contribution in the red wing from strong $\mathrm{H} \alpha$ line emission. However, the difference in filters cannot explain the poor agreement between the data and the relationship in Figure 9 since there are similar numbers of novae that fall above the line as below. If a contribution from $\mathrm{H} \alpha$ in $V$ was significant then the disagreement would not be symmetric.

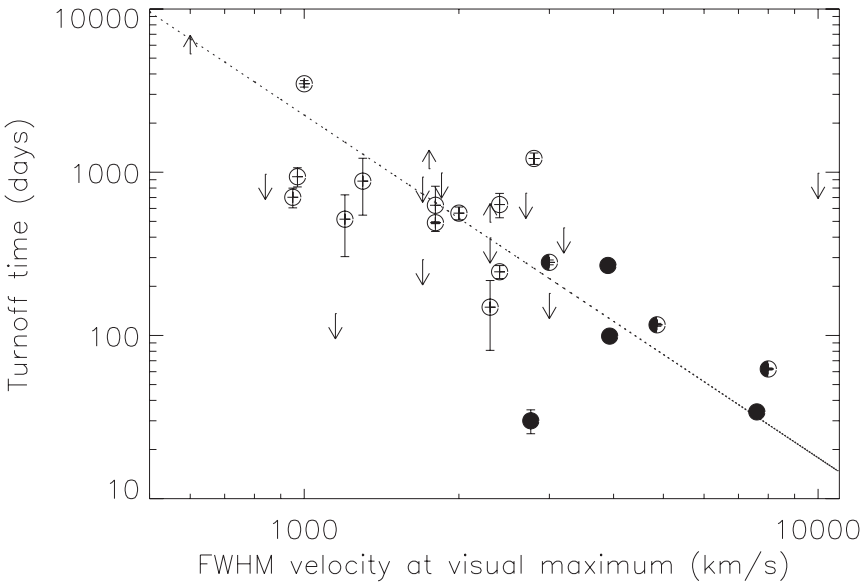

Figure 10. SSS turn-off time as a function of the FWHM of $\mathrm{H} \alpha$ or $\mathrm{H} \beta$ near visual maximum. The relationship of Greiner et al. (2003) is shown as the dotted line. Upper and lower limits are also shown. Filled circles are known RNe. Half filled circles are suspected RNe based on their characteristics.

Similarly, Figure 10 shows the relationship between the FWHM and turn-off time with the dotted line depicting the Greiner et al. (2003) turn-off versus velocity relation. This relationship was derived from all the SSS nova data available at the time, which consisted of only four well-constrained SSS novae and four novae with turnoff limits. With the significantly larger sample currently available, it is clear that there is not a tight fit to the relationship. This discrepancy is particularly acute for the slower novae in our sample, which have turned off much sooner than expected. These figures illustrate that the gross behavior of novae is still poorly understood and confirm that the observational characteristics of an individual nova is governed by more than just the WD mass.

Accurate determinations of the duration of nuclear burning can also provide an independent ejected mass estimate. 
Table 5

SSS X-Ray Timescales

\begin{tabular}{|c|c|c|}
\hline Name & $\begin{array}{c}\text { Turn-on }{ }^{\mathrm{a}} \\
\text { (days) }\end{array}$ & $\begin{array}{c}\text { Turn-off } \\
\text { (days) }\end{array}$ \\
\hline CI Aql & $\ldots$ & $<396$ \\
\hline CSS 081007 & $185 \pm 68$ & $314 \pm 68$ \\
\hline GQ Mus & $\ldots$ & $3484.5 \pm 159.5$ \\
\hline IM Nor & $>28$ & $<136$ \\
\hline KT Eri & $71 \pm 1$ & $280 \pm 10$ \\
\hline LMC 1995 & $<1087$ & $2545 \pm 426$ \\
\hline LMC 2000 & $>48$ & $<291$ \\
\hline LMC 2009a & $95 \pm 5$ & $270 \pm 10$ \\
\hline RS Oph & $35 \pm 5$ & $70 \pm 2$ \\
\hline U Sco & $23 \pm 1$ & $34 \pm 1$ \\
\hline V1047 Cen & $>144$ & $<972$ \\
\hline V1065 Cen & $\ldots$ & $<744^{\mathrm{b}}$ \\
\hline V1187 Sco & $\ldots$ & $<181^{\mathrm{b}}$ \\
\hline V1213 Cen & $<322$ & $>494$ \\
\hline V1280 Sco & $>928$ & $\ldots$ \\
\hline V1281 Sco & $<339$ & $627 \pm 194$ \\
\hline V1494 Aql & $217.5 \pm 30.5$ & $515.5 \pm 211.5$ \\
\hline V1974 Cyg & $201 \pm 54$ & $561.5 \pm 50.5$ \\
\hline V2361 Cyg & $\ldots$ & $<456$ \\
\hline V2362 Cyg & $\ldots$ & $<990$ \\
\hline V2467 Cyg & $<456$ & $702 \pm 97$ \\
\hline V2468 Cyg & $<586$ & $\ldots$ \\
\hline V2487 Oph & $\ldots$ & $<986$ \\
\hline V2491 Cyg & $40 \pm 2$ & $44 \pm 1$ \\
\hline V2672 Oph & $22 \pm 2$ & $28 \pm 2$ \\
\hline V351 Pup & $\ldots$ & $<490$ \\
\hline V382 Vel & $<185$ & $245.5 \pm 22.5$ \\
\hline V407 Cyg & $15 \pm 5$ & $30 \pm 5$ \\
\hline V458 Vul & $406 \pm 4$ & $>1051$ \\
\hline V4633 Sgr & $\ldots$ & $<934$ \\
\hline V4743 Sgr & $115 \pm 65$ & $634 \pm 108$ \\
\hline V5114 Sgr & $<1086$ & $\ldots$ \\
\hline V5115 Sgr & $<546$ & $882 \pm 336$ \\
\hline V5116 Sgr & $332.75 \pm 275.25$ & $938 \pm 126$ \\
\hline V5558 Sgr & $>850^{\mathrm{c}}$ & $\ldots$ \\
\hline V5583 Sgr & $<81$ & $149 \pm 68$ \\
\hline V574 Pup & $571 \pm 302$ & $1192.5 \pm 82.5$ \\
\hline V597 Pup & $143 \pm 23$ & $455 \pm 15$ \\
\hline V598 Pup & $\ldots$ & $<127$ \\
\hline V723 Cas & $<3698$ & $>5308$ \\
\hline V838 Her & $\ldots$ & $<365$ \\
\hline XMMSL1 J060636 & $\ldots$ & $<291$ \\
\hline
\end{tabular}

Notes. $t_{\mathrm{on}}$ and $t_{\mathrm{off}}$ bracket the time after visual maximum when the hardness ratio HR2 is softer than -0.8 .

a Time since visual maximum, see Table 1 .

b Evolution of [Fe VII] (6087 $\AA$ ) and lack of [Fe x] (6375 $\AA$ ) in our SMARTS optical spectra are consistent with this upper limit from the X-ray non-detection. See Section 5.6.

c Optical spectra are slowly becoming more ionized which is consistent with slowly increasing SSS emission observed with Swift.

Recently, Shara et al. (2010) found that the ejected mass is only dependent on the total radiated energy, $E_{\mathrm{rad}}$, and does not require knowledge about the geometry and structure of the shell as with other methods. $E_{\text {rad }}$ is not a trivial value to determine as it depends on the bolometric luminosity of the source and the duration of the outburst. Swift observations can potentially determine the bolometric flux both when the bulk of the emission is in a narrow wavelength region such as the early, optically thick phases in the UV and optical or later in the soft X-ray during the SSS phase. Estimates of the luminosity during both phases require an accurate determination of the extinction and the distance. Perhaps the best example to use the Shara et al. (2010) technique on is RS Oph. With a bolometric luminosity of $3 \times 10^{4} L_{\odot}$ from the Tübingen NLTE Model-Atmosphere Package (Osborne et al. 2011) and a $t_{\text {off }}$ of 70 days, the estimated ejected mass $\sim 2 \times 10^{-6} M_{\odot}$. This is consistent with the low mass estimates from the radio $\left((4 \pm 2) \times 10^{-7} M_{\odot}\right.$; Eyres et al. 2009) and hydrodynamical models of the X-ray behavior $\left(1.1 \times 10^{-6} M_{\odot}\right.$; O'Brien et al. 1992 and $\sim 5 \times 10^{-6} M_{\odot}$; Drake et al. 2009).

\subsubsection{SSS Phase Durations}

Figure 11(left) shows the distribution of the duration of the SSS phase for this sample of novae. Since there are still relatively few novae with well-established turn-off times, a coarse histogram with only three bins is used. The bins have durations of less than one year, between one and three years, and greater than three years. Due to large uncertainties in their exact turn-off times, 10 of the sample novae cannot be placed within a single bin and thus are shown as the smaller crosshatched columns between the bins in which they might belong. Of the 38 novae with detected SSS emission or with strong limits on the duration of the SSS phase, $89 \%$, have turned off in under three years. There are only four novae, GQ Mus, LMC 1995, V574 Pup, and V723 Cas, with detected SSS emission beyond three years. V458 Vul and V1213 Cen were still SSSs at their last observations and could also exceed three years. A similar rapid turn-off was inferred from a search of the ROSAT archive of novae with SSS detections. Orio et al. (2001a) found only three SSS novae among the 39 Galactic and Magellanic Cloud novae in the ROSAT archive observed at least once within 10 years after visual maximum. The median age of the 19 novae with documented turn-off times from this sample is 1.4 years.

The situation is different for nova surveys of M31 (Pietsch et al. 2007; Henze et al. 2010) where SSSs identified as CNe 5-10 years after outburst are fairly common, e.g., 1995-05b, 1995-11c, and 1999-10a. Figure 11(right) shows the same bins as before but with the $18 \mathrm{M} 31$ novae detected as SSSs given in Table 9 of Henze et al. (2010). The difference can be explained by the predominance of slower novae in the M31 sample. The mean $t_{2}$ time of the nine M31 novae with reported decline times in the Henze et al. (2010) SSS sample is 31 days whereas the peak for our sample is significantly faster at eight days (Figure 1). The discrepancy in speed class between the two samples is due to selection effects. By design the Galactic/ Magellanic sample consists primarily of bright and hence faster novae. M31 surveys sample the entire galaxy but with fewer Chandra, XMM, and Swift observations that are randomly scattered in time. The M31 strategy finds many novae since the observed M31 nova rate is greater $\left(\sim 30\right.$ novae year $^{-1}$; Capaccioli et al. 1989) than that of the Milky Way ( $\sim 5$ novae year ${ }^{-1}$; Shafter 1997), however, with limited time sampling slower novae with longer SSS phases are easier to detect than fast novae with rapid turn-offs.

ROSAT detected two SSS novae out of 21 Galactic novae for a Milky Way detection frequency of $9.5 \%$ (Orio et al. 2001a). If the four $\mathrm{RNe}$ in the ROSAT list are discarded because their observations were taken $\gtrsim 1$ year after outburst, the detection frequency increases to $11.8 \%$. The M31 survey has a similar low SSS detection frequency of 6.5\% (Pietsch 2010). These two results show that it is difficult to catch novae during their SSS phase via random time sampling. However, a more systematic approach that (1) targets only bright and low-extinction novae and (2) obtains multiple observations early in the outburst 

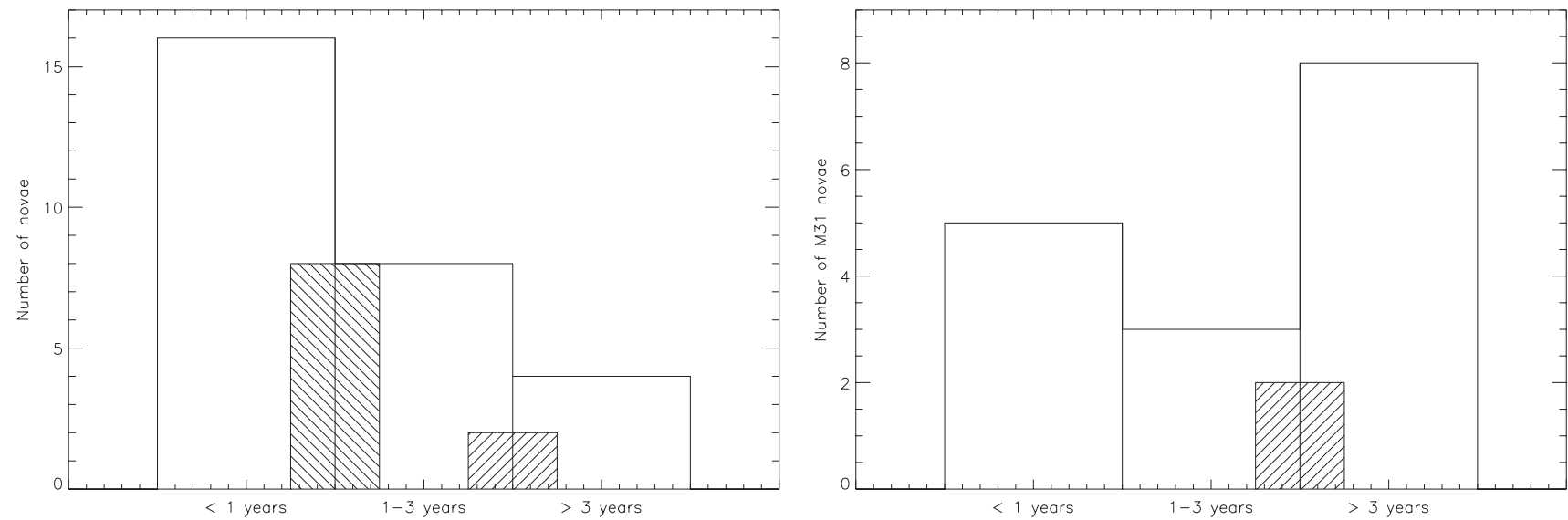

Figure 11. Distribution of the durations of well-established SSS novae in the Galaxy/Magellanic Clouds (left figure) and M31 (right figure) from Henze et al. (2010). The three duration bins are less than one year, between and and three years, and greater than three years. The hatched areas include the novae with only limits on their turnoff time that precludes placing them in a specific bin.
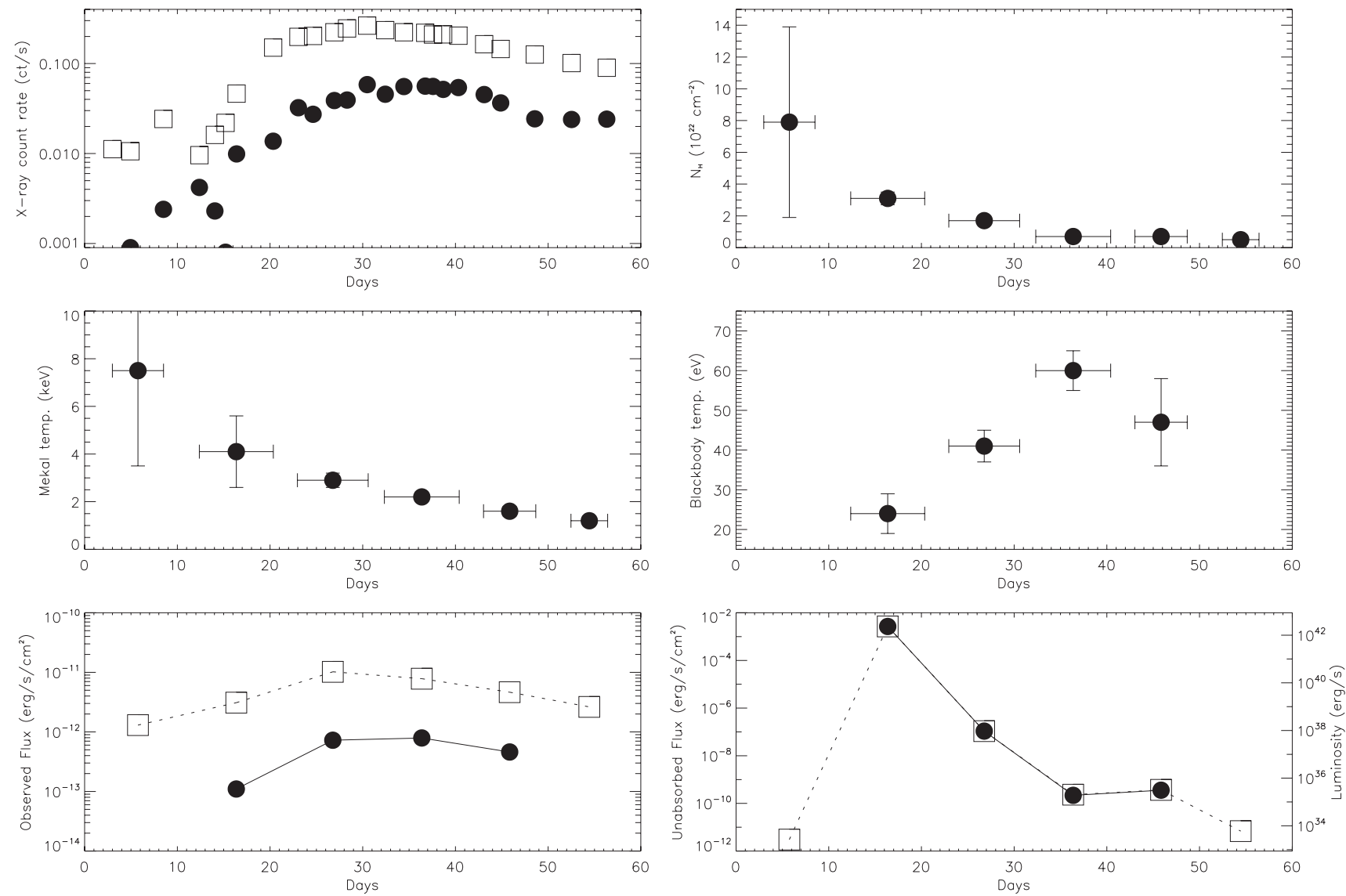

Figure 12. Results of model fits to the Swift V407 Cyg data set. The top left panel shows the total $0.3-10 \mathrm{keV}$ (squares) and soft $0.3-1 \mathrm{keV}$ (circles) light curves. The derived $N_{\mathrm{H}}$ column for the six date bins is shown in the top right panel. The Mekal temperature of the hotter, optical thin plasma model is shown in the middle left panel while the right middle panel shows the temperature of the blackbody fit to the softer component. A second, soft component is not needed in the first and last date bins. The bottom panels show the observed (left) and unabsorbed (right) fluxes. Squares give the total from all components while the circles show just the blackbody contributions. The right axis of the last panel also shows the corresponding $0.3-10 \mathrm{keV}$ luminosity assuming a $2.7 \mathrm{kpc}$ distance.

may have a greater detection frequency. Indeed, Swift has a significantly greater SSS detection rate of $\sim 45 \%$ during its five years of operation with this more systematic approach.

\subsection{SSS Emission in the Hard X-Ray Spectrum of V407 Cyg}

In the initial analysis of the Swift data in Shore et al. (2011) a second soft component was required to fit some of the Swift X-ray spectra. However, there were insufficient counts to distinguish between a blackbody and an optically thin plasma model. Assuming a distance of $2.7 \mathrm{kpc}$ (Munari et al. 1990), the unabsorbed flux of the soft component in the day $<30$ model of Table 3 in Shore et al. (2011) gives a blackbody luminosity of $2 \times 10^{37} \mathrm{erg} \mathrm{s}^{-1}$ which is reasonable for nuclear burning on a WD. To investigate whether the soft emission in V407 Cyg can be attributed to nuclear burning, we reanalyze the Swift $\mathrm{X}$-ray data with twice as many time bins as previously used. Figure 12 shows the results. As in Shore et al. (2011), the model abundances are allowed to vary but the temperatures are not 


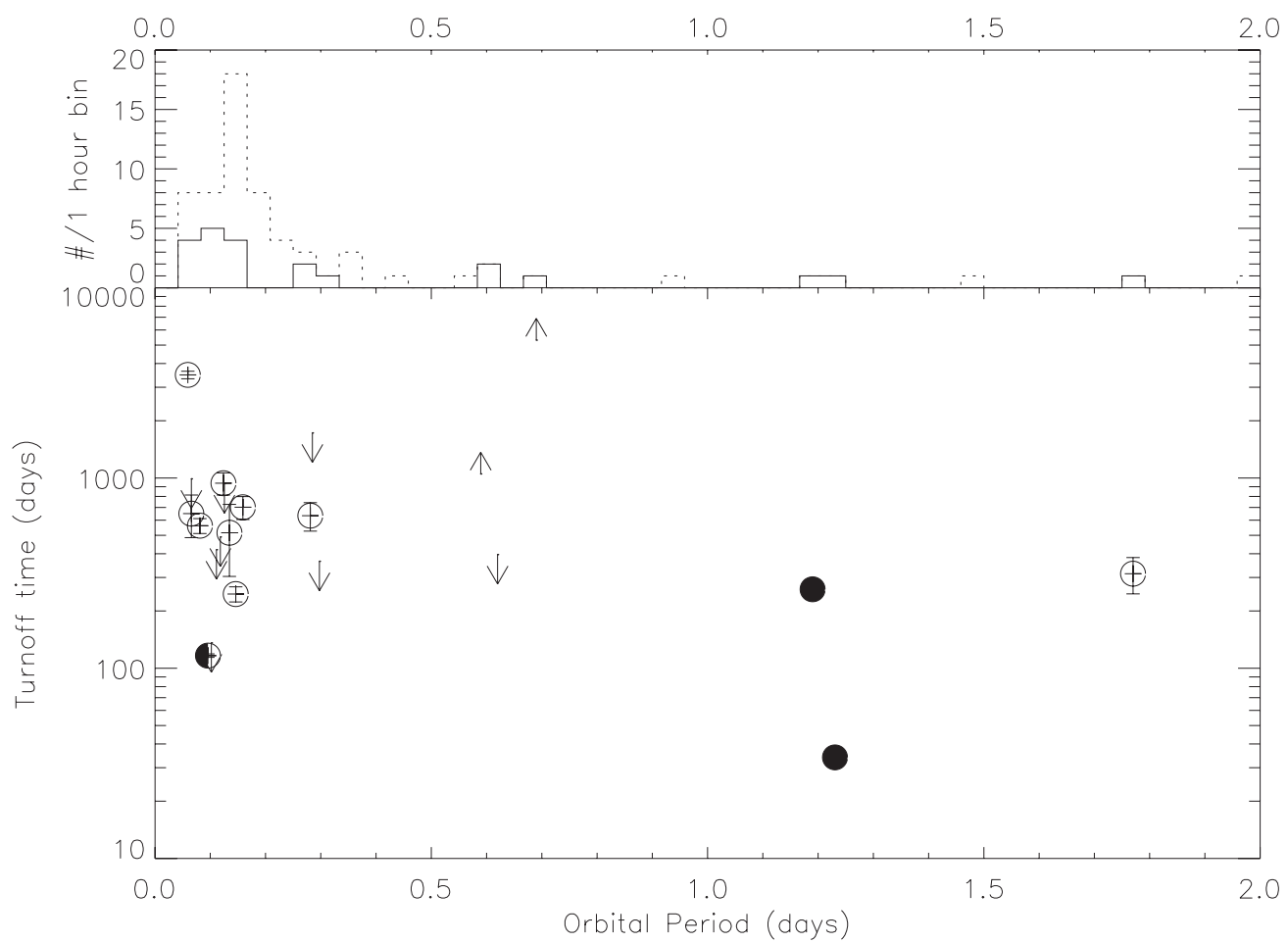

Figure 13. SSS turn-off time as a function of orbital period for novae with well-established turn-off times and novae with good upper (i.e., still in the SSS phase) and lower limits. Filled circles are known RNe. Half filled circles are suspected RNe based on their characteristics. The top plot shows the distribution histogram of our sample (solid line) and of all the known novae (dotted line) from Table 2.5 in Warner (2008).

significantly different if the abundances are constrained to be solar. The data prior to day 10 and after day 50 can be fit with a single optically thin plasma model. The remaining four time bins all require a soft component, which in this analysis is assumed to be a blackbody. Both the derived $N_{\mathrm{H}}$ and the optically thin component temperature decline with time in the models. The blackbody effective temperature increases until the day 36 bin and declines in the day 45 bin, although the error bars are large enough that it could be constant over the last two dates. The derived luminosities (over the $0.3-10 \mathrm{keV}$ $\mathrm{X}$-ray band) for the four dates with blackbody components are $2.3 \times 10^{42} \mathrm{erg} \mathrm{s}^{-1}, 9.3 \times 10^{37} \mathrm{erg} \mathrm{s}^{-1}, 1.9 \times 10^{35} \mathrm{erg} \mathrm{s}^{-1}$, and $3.1 \times 10^{35} \mathrm{erg} \mathrm{s}^{-1}$, respectively, assuming a distance of $2.7 \mathrm{kpc}$. The extreme luminosity for the day 16 bin cannot be considered reliable, given the very low fitted temperature of $\sim 25 \mathrm{eV}$ below the XRT $0.3 \mathrm{keV}$ low-energy cutoff. Nevertheless, the results of fitting blackbodies to the Swift V407 Cyg data are consistent with a scenario where the nuclear burning proceeded on the WD surface near Eddington limits until about 30 days after visual maximum. The fuel was consumed after that point leading to a rapid drop in the luminosity. Thus, although V407 Cyg was not a true SSS, its soft photon light curve was consistent with expected evolution as seen in other novae.

\section{DISCUSSION}

\subsection{Orbital Period and Turn-off Time}

Greiner et al. (2003) found a correlation between the orbital period and X-ray turn-off time. However, at that time only four novae had both well-determined periods and X-ray turnoff times, GQ Mus, V1974 Cyg, V1494 Aql, and V382 Vel, and limits on CI Aql and U Sco. The observed trend implied that novae with short orbital periods had the longest duration SSS phases. Greiner et al. (2003) attributed this relationship to a feedback loop between the WD and its secondary. The luminous X-rays produced during the SSS phase excessively heat the facing side of the secondary in short-period systems. The energy added to the outer layers of the secondary causes it to expand, producing higher mass loss leading to enhanced accretion of material onto the WD.

Since 2003, the turn-off times of 10 additional novae with known periods have been determined. There are also strong limits on the turn-off times of 10 other novae with known orbital periods. Inclusion of this expanded sample, shown in Figure 13, causes the trend between orbital period and duration of the SSS phase noted by Greiner et al. (2003) to disappear. The new distribution, with an increased sample size, shows no discernible correlation. Orbital separation apparently has no effect on the duration of nuclear burning.

To see if the lack of a trend could be explained by having a non-representative sample of novae, the top panel of Figure 13 shows the distribution in $1 \mathrm{hr}$ orbital period bins of the updated Warner (2008) sample as the solid line. The distribution of all novae with orbital periods is shown as the dotted line and shows that the SSS sample is a consistent sub-sample of the known nova period distribution.

Schaefer \& Collazzi (2010) claim a similar relationship between turn-off time and orbital period, albeit in highly magnetized systems. They find that of the eight novae with quiescent luminosities $>10 \times$ brighter than pre-eruption, all have long SSS phases, short orbital periods, highly magnetized WDs, and very slow declines during quiescence. Similar to Greiner et al. (2003), Schaefer \& Collazzi (2010) propose that nuclear burning on the WD is prolonged by increased accretion from the close secondary but in this case efficiently funneled on the WD by the strong magnetic fields. The eight novae (Schaefer \& Collazzi 2010) cited are CP Pup, RW UMi, T Pyx, V1500 Cyg, GQ Mus, V1974 Cyg, V723 Cas, and V4633 Sgr. 
The hypothesis that these specific characteristics enhance the SSS duration can be directly evaluated using V4633 Sgr, GQ Mus, V1974 Cyg, and V723 Cas, since they all have X-ray observations within the first three years of outburst. For CP Pup, RW UMi, T Pyx, and V1500 Cyg the assertion of a long-lasting SSS emission phase depends on secondary evidence as none had any direct X-ray observations during outburst. Lacking direct X-ray observations, we will ignore these four sources for the test.

The first X-ray observation of V4633 Sgr was obtained 934 days after visual maximum but it and subsequent observations were of a hard source implying that any SSS emission was missed. With an upper limit of 2.5 years for its SSS emission, V4633 Sgr cannot be considered a long-lived SSS nova based on the distribution shown in Figure 11 (left). The SSS duration in V1974 Cyg was even shorter and much better constrained at $1.53 \pm 0.14$ years. In addition, V1974 Cyg was not "excessively" luminous in outburst as alleged in Schaefer \& Collazzi (2010). Its early UV plus optical fluxes were consistent with the Eddington luminosity of a WD with a mass range of $0.9-1.4 M_{\odot}$ (Shore et al. 1994). The later "excessive" X-ray luminosities of Balman et al. (1998) were derived from blackbody fits, which are known to predict higher luminosities than model atmospheres fit to the same data. While V723 Cas has the longest SSS duration known among novae ( $\gtrsim 15$ years), its orbital period is very long at $16.62 \mathrm{hr}$ and significantly longer than that of GQ Mus, $1.43 \mathrm{hr}$. The claim of magnetic activity in V723 Cas is based on the different periodicities observed in the early light curve indicating an intermediate polar (IP). However, the multiple periodicities used as evidence by Schaefer \& Collazzi (2010) were from data obtained early in the outburst while the nova ejecta were still clearing (Chochol \& Pribulla 1998). Photometry obtained at this early stage of development frequently results in noisy periodograms. Data obtained later in the outburst by Goranskij et al. (2007) and over the last four years from our own photometric monitoring (C. Hamilton 2011, private communication; Schwarz et al. 2007a) reveal a well-defined $16.7 \mathrm{hr}$ period with a large $\sim 1.5$ mag amplitude in the UV, optical, and NIR bands. There is no other evidence in the literature to support that V723 Cas is magnetic. Of the four novae with supporting X-ray observations, only GQ Mus fully matches the criteria of a long-lasting SSS on a magnetic WD in a short-period system.

With our expanded X-ray sample there are three additional novae with well-constrained SSS durations that can potentially be used to test the hypothesis. V4743 Sgr (Kang et al. 2006), V597 Pup (Warner \& Woudt 2009), and V2467 Cyg (Swierczynski et al. 2008) are IP candidates and thus believed to have strong magnetic fields. The orbital periods for V597 Pup and V2467 Cyg are relatively short at 2.66 and $3.8 \mathrm{hr}$, respectively, but the period in V4743 Sgr is much longer at $6.74 \mathrm{hr}$, see Table 1. While the turn-off times for these novae are all longer than one year they are not exceptionally long, with durations of $1.74 \pm 0.29,1.25 \pm 0.04$, and $1.85 \pm 0.33$ years for V4743 Sgr, V597 Pup, and V2467 Cyg, respectively. Thus the data available do not imply that short orbital period or strong magnetic fields produce significantly longer SSS than the average novae from our sample.

An interesting question is why there is no trend between orbital period and SSS duration as the underlying assumption of enhanced accretion due to heating of the secondary is sound. One reason would be that there is no significant enhancement in the mass transfer rate from the illuminated secondary, perhaps from shielding due to a thick disk. Another possibility is that there is an effect but it is subtle and affected by other variables such as the strength of the magnetic field, composition of the accreted material, WD mass, etc. Another possibility is that an accretion disk cannot form under the harsh conditions during the SSS phase which inhibits additional mass transfer. More observations of novae with different characteristics are required in order to understand the underlying physics.

\subsection{Dusty Novae}

The creation, evolution, and eventual destruction of dust occurs on relatively rapid timescales in novae making them excellent objects for understanding dust grain formation. One curious aspect of dust in novae is how grains can grow within the harsh photoionizing environment. A correlation of the recent Spitzer spectroscopic observations of dusty novae (see Woodward \& Starrfield 2011; Helton 2010, for examples) with this large X-ray sample can bring insights to why most novae do not form dust and the reasons for the large differences in composition and amounts in the novae that do form dust.

In general, it is believed that grain growth occurs within dense clumps in the ejecta where they are shielded from hard radiation. Spectroscopic and direct imaging show that nova shells are inherently clumpy (Shara et al. 1997; O'Brien \& Bode 2008). Grain formation inside dense clumps also explains the higher frequency of dust in slow novae (see Table 13.1 in Evans \& Rawlings 2008) as they eject more material at lower velocities and suffer greater remnant shaping than fast novae and thus provide more protection for grain formation. However, even fast novae with small ejected masses have shown some dust formation, such as V838 Her (Schwarz et al. 2007b). A contrary view was proposed that ionization actually promotes dust formation via the accretion of grain clusters through induced dipole interactions (Shore \& Gehrz 2004).

Known and likely dusty novae represent $16 \%$ of the X-ray sample but only two, V2467 Cyg and V574 Pup, were also SSSs. While there were no characteristic dips in either visual light curve indicating significant dust formation (Lynch et al. 2009; Siviero et al. 2005), both novae showed evidence of some dust formation from the presence of weak silicate emission features in the late Spitzer mid-IR spectra (Woodward \& Starrfield 2011). In V2467 Cyg the first Swift X-ray detection was 458 days after maximum. It was weak but dominated by soft photons. The following Swift observation on day 558 revealed the nova was still soft but was also almost three times brighter. The Spitzer spectra showing weak dust features were taken between these Swift observations, around day 480. V574 Pup was detected as an SSS by XMM and Swift 872 and 1116 days after visual maximum, respectively. Spitzer observations taken around the same time as the Swift data showed the same weak silicate emission features seen in V2467 Cyg. The X-ray observations confirm that dust, albeit weak, can exist in the ejecta when the amount of photoionizing radiation is at its peak. Detailed photoionization modeling of these novae is required to determine if clumps existed in the ejecta during this time and if the conditions were sufficient to protect the dust grains.

There are also two strong dust formers in the sample with hard X-ray emission. V2362 Cyg was detected numerous times by Swift (Lynch et al. 2008) and twice with XMM (Hernanz et al. 2007) but none of the observations were consistent with an SSS. However, V2362 Cyg had significant dust emission at the times of the Swift and first XMM observations. The dust likely formed 


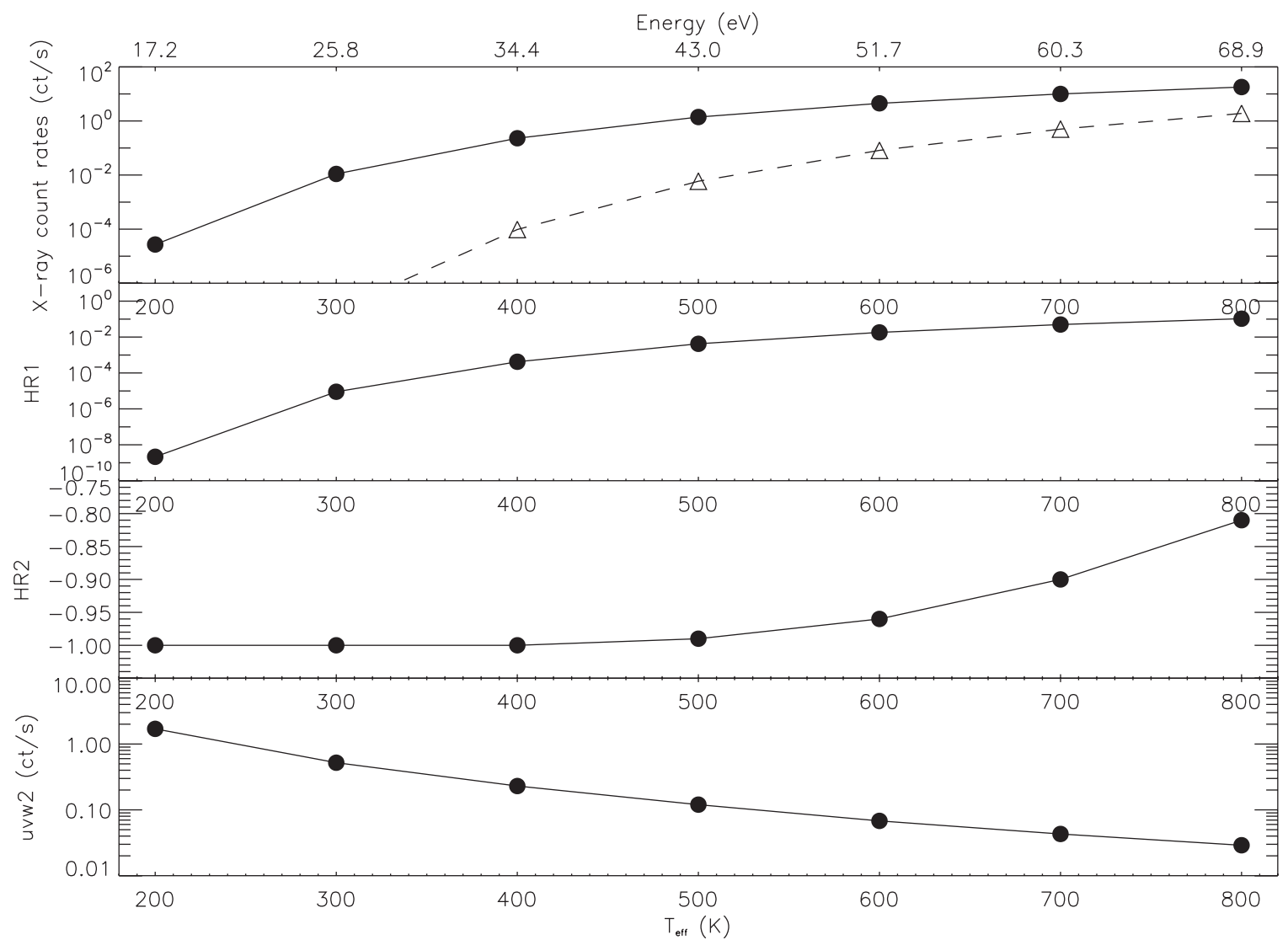

Figure 14. Logarithmic X-ray count rates, hardness ratios, and logarithmic uvw2 count rates as a function of blackbody temperature as calculated by WebPIMMS. An unabsorbed, bolometric flux of $3.3 \times 10^{-8} \mathrm{erg} \mathrm{s}^{-1} \mathrm{~cm}^{-2}\left(1 \times 10^{38} \mathrm{erg} \mathrm{s}^{-1}\right.$ at $\left.5 \mathrm{kpc}\right)$ and $N_{\mathrm{H}}$ of $3 \times 10^{21} \mathrm{~cm}^{-2}$ was used in all models. The top panel shows the soft $(0.3-1 \mathrm{keV}$, solid line and filled circles) and hard $(0.1-10 \mathrm{keV}$, dashed line and triangles) count rates. The soft contribution dominates at all effective temperatures. The middle panels show the hardness ratios HR1 $(=H / S)$ and $\mathrm{HR} 2(=(H-S) /(H+S))$. The bottom panel shows how the uvw2 count rate increases as the blackbody temperature declines.

in the later extraordinary mass ejection event that produced the large secondary peak in the light curve and increased ejection velocities. The additional material would have absorbed the soft X-ray emission and delayed the onset of any SSS phase. In the last XMM observation it was extremely faint indicating that if there was an SSS phase it was over by 990 days after maximum. V1280 Sco was detected as an X-ray source late in its outburst but the X-rays were relatively hard and faint (Ness et al. 2009b). V1280 Sco has yet to be observed as an SSS and its internal extinction is still large. In both V2362 Cyg and V1280 Sco, grain growth was likely enhanced to produce the large dust events due to the effective shielding of the large mass ejections.

\subsection{Variability during SSS Phase}

At the maximum effective temperature, $(2-8) \times 10^{5} \mathrm{~K}$, the bulk of the emission in a nova outburst comes from X-rays that are primarily soft. Assuming the external column is low enough and the effective temperature is suitably high enough, this X-ray emission can be detected. The theory of constant bolometric luminosity predicts that at X-ray maximum the light curve should be relatively constant since one is observing the majority of the emitted flux. Constant bolometric luminosity has been observationally verified in the early phase of the outburst from the combined UV, optical, and near-IR light data, e.g., FH Ser (Gallagher \& Code 1974), V1668 Cyg (Stickland et al. 1981), and LMC 1988\#1 (Schwarz et al. 1998). However, the expected X-ray plateau in all well-studied Swift novae has been far from constant. In addition, the rise to X-ray maximum also shows large amplitude oscillations. What is the source of the variability during both phases?

One important caveat when discussing the Swift data is that the XRT count rate is not a direct measure of the bolometric flux, only the portion that is emitted between 0.3 and $10 \mathrm{keV}$. During the SSS phase the vast majority of photons are emitted within this range, but if the effective temperature varies due to photospheric expansion or contraction, the XRT count rate will change even if the source has a constant bolometric luminosity (see also Osborne et al. 2011). Figure 14 illustrates how the estimated XRT count rate varies as a function of effective temperature for simple blackbody models (WebPIMMS ${ }^{13}$ ) assuming a constant luminosity and column density see Section 5.3.1. A decline from $500,000 \mathrm{~K}$ to $400,000 \mathrm{~K}$ drops the total Swift XRT count rate by a factor of six. The change in HR1 is almost a factor of 10 , while there is essentially no change in the HR2 hardness ratio. Thus changes in temperature might in principle account for the observed X-ray oscillations, see Section 5.3.3. Why the temperature or radius of the WD photosphere would change on the observed timescales remains an open question however. The next sections outline possible explanations for the variations seen during the SSS phase.

\subsubsection{Variable Visibility of the WD}

Figures 2 and 5 in Osborne et al. (2011) show in exquisite detail the rapid and extreme variability in the X-ray light curve

\footnotetext{
13 http://heasarc.nasa.gov/Tools/w3pimms.html
} 
and hardness ratio evolution in RS Oph. In general the trend was for RS Oph to be softer at high X-ray flux but counter examples were also observed. Osborne et al. (2011) cite variable visibility of the hot WD as a possible explanation of the observed phenomena. Changes in the extinction can come from either variable ionization of the ejecta leading to changing extinction at higher ionization states or neutral absorption from high density clumps passing through the line of sight. Changes in the ionization structure of the ejecta are unlikely given the rapid hour to day timescales but are consistent with the crossing times of small, dense clumps traveling across the line of sight assuming transverse velocities of a few percent of the radial velocity. There is evidence for this at other wavelengths. For example, a sudden absorption component that appeared in the Balmer lines of V2214 Oph in 1988 July was interpreted by Williams et al. (1991) as the passage of an absorbing clump in front of the emitting region. Both types of absorption changes should be manifest as a hardening of the X-ray spectrum or increase in the hardness ratio with increasing soft flux emission consistent with the counter examples of Osborne et al. (2011).

As a test of the neutral absorption theory we use the model results from a recent photoionization analyses in WebPIMMS to determine the count rates and hardness ratios for different column densities and simulate the effect of clumps. The photoionization models require two components, high density clumps embedded within a larger diffuse medium (see Schwarz et al. 2007b; Helton et al. 2010 for details) to fit the emission lines of the ejected shell. For convenience we use the 1991 May 24th model parameters for V838 Her in Table 2 of Schwarz et al. (2007b). The model uses a blackbody with an effective temperature of $200,000 \mathrm{~K}$ to photoionize a two component spherical shell. The model shell has a clump-to-diffuse density ratio of 3 with a radius equal to the expansion velocity multiplied by the time since outburst. To facilitate comparisons with the results in Figure 14, the same unabsorbed bolometric flux is assumed. WebPIMMS predicts a Swift soft band count rate of $5.3 \times 10^{-3}$ counts $\mathrm{s}^{-1}$ through the lower density diffuse gas $\left(N_{\mathrm{H}}=1.2 \times 10^{21} \mathrm{~cm}^{-2}\right)$ and $8.5 \times 10^{-6}$ counts $\mathrm{s}^{-1}$ from the higher density clumps $\left(N_{\mathrm{H}}=3.7 \times 10^{21} \mathrm{~cm}^{-2}\right)$. While the total count rate declines by over $100 \times$, the $\mathrm{HR} 2$ hardness ratio does not change with this particular model. The HR2 can vary significantly when using different model parameters such as lower initial densities or higher clump to diffuse density ratios. Care is required when using hardness ratios of low-resolution data. In an SSS source with a hard X-ray component, such as RS Oph, the hardness ratio will increase if the soft component decreases for any reason, not just due to absorption.

Another problem with variable visibility in $\mathrm{RNe}$ and very fast $\mathrm{CNe}$ is that the amount of mass ejected is very low thus minimizing any effect the ejecta have on the obscuration of the WD. The effect should be greater in slower novae with more ejected mass such as V458 Vul. In addition, Osborne et al. (2011) find that in RS Oph the ratio of high flux states to low flux states as a function of energy is not consistent with either type of variable visibility of the WD. Rather the best fit comes from an increase in the effective temperature and declining radius, see Section 5.3.3.

\subsubsection{Periodic Oscillations}

There are several proposed explanations of the periodic X-ray variations. In the X-ray light curve of V1494 Aql, Drake et al. (2003) found periodicities that they attributed to non-radial $g^{+}$-mode pulsations. Similar oscillations have been observed in V4743 Sgr (Ness et al. 2003; Dobrotka \& Ness 2010).

The factor of almost 10 decline in the $X M M X$-ray light curve of V5116 Sgr was interpreted by Sala et al. (2008) as a partial eclipse of the WD since its duration was consistent with the orbital period. Finer binning of the day 762, 764, and 810 Swift observations of V5116 Sgr reveals the presence of a 500-800 s oscillation. This X-ray periodicity is significantly shorter than the $2.97 \mathrm{hr}$ orbital period found by Dobrotka et al. (2008). In addition, the day 810 data show a strong flare that increases the count rate by a factor of three with no significant change in the hardness ratio. This was similar to the flare seen in V1494 Aql (Drake et al. 2003). No other flares were seen in the V5116 Sgr data set.

Other orbital periods have been detected with Swift. U Sco is a high inclination system with deep eclipses and an orbital period of 1.23 days (Evans et al. 2001). Deep eclipses were observed in the 2010 outburst in the Swift UVOT light curves while the XRT light curves showed generally lower flux levels during the UV eclipses, but did not otherwise exhibit clear eclipse signatures (Osborne et al. 2010). A 1.19 day orbital period was deduced from the Swift UVOT light curves in the RN nova LMC 2009a (Bode et al. 2009a). This orbital period was also observed in the XRT light curve during the SSS phase, but with a lag with respect to the UV/optical of 0.24 days (M. F. Bode et al. 2011, in preparation).

The X-ray behavior in CSS 081007:030559+054715 was extremely unusual. This odd source was discovered well after optical maximum by the Catalina Real-time Transient Survey (Prieto et al. 2008). Its X-ray spectra were extremely soft, consistent with the low extinction along its position high above the Galactic plane $(b=-43.7)$, which places it well outside the plane of the Galaxy where novae are generally not located. Figure 15 shows the Swift XRT/UVOT light curves compiled from the data in Table 2. To first order both light curves are in phase with significant variability superimposed over three major maxima. Beardmore et al. (2010b) report that the Swift light curves are unique with a 1.77 day periodicity. They speculate that the period is due to obscuration of the X-ray source in a high inclination system with a 1.77 day orbital period.

Oscillations significantly shorter than the hours to days of typical novae orbital periods have also been detected with Swift. Oscillations of order $35 \mathrm{~s}$ have been observed in RS Oph (Osborne et al. 2006, 2011) and KT Eri (Beardmore et al. 2010a). Some WDs have rotation periods in this range (e.g., $33 \mathrm{~s}$ in AE Aqr; Terada et al. 2008). It seems unlikely that RS Oph and KT Eri should both have nearly identical rotation periods unless the pulsations are tied to the mass of the WD which for both novae are predicted near the Chandrasekhar limit. Another reason the observed variability might not be associated with the rotating WD is that the $\sim 35$ s periodicity is not always detected in the Swift and Chandra X-ray light curves. The $35 \mathrm{~s}$ pulsations could be due to a nuclear burning instability on the WD surface (see Osborne et al. 2011). If so, then the period then is a function of WD mass, and perhaps indicates that the WDs in RS Oph and KT Eri are near the Chandrasekhar mass.

\subsubsection{Temperature Variations}

Long-lived SSS, such as Cal 83, have non-periodic X-ray on/off states. Reinsch et al. (2000) speculate that the decline in X-ray flux is due to accretion disk interactions such as an increase in the mass accretion rate causing the WD photosphere to expand and shifting the SED into the EUV. These sources then 


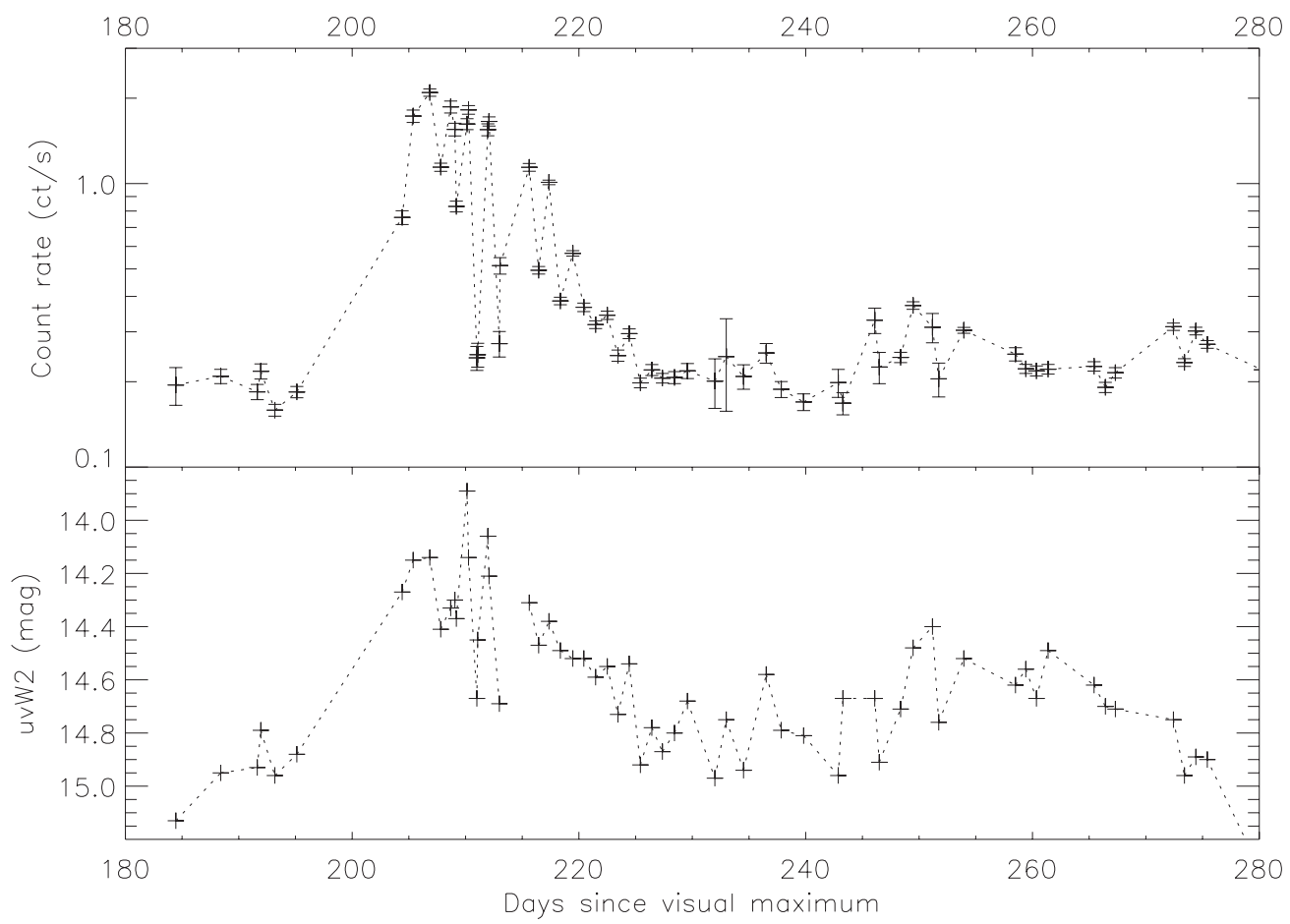

Figure 15. X-ray and uvw2 light curves of the particular nova CSS 081007:030559+054715. The X-ray and UV evolution are in phase.

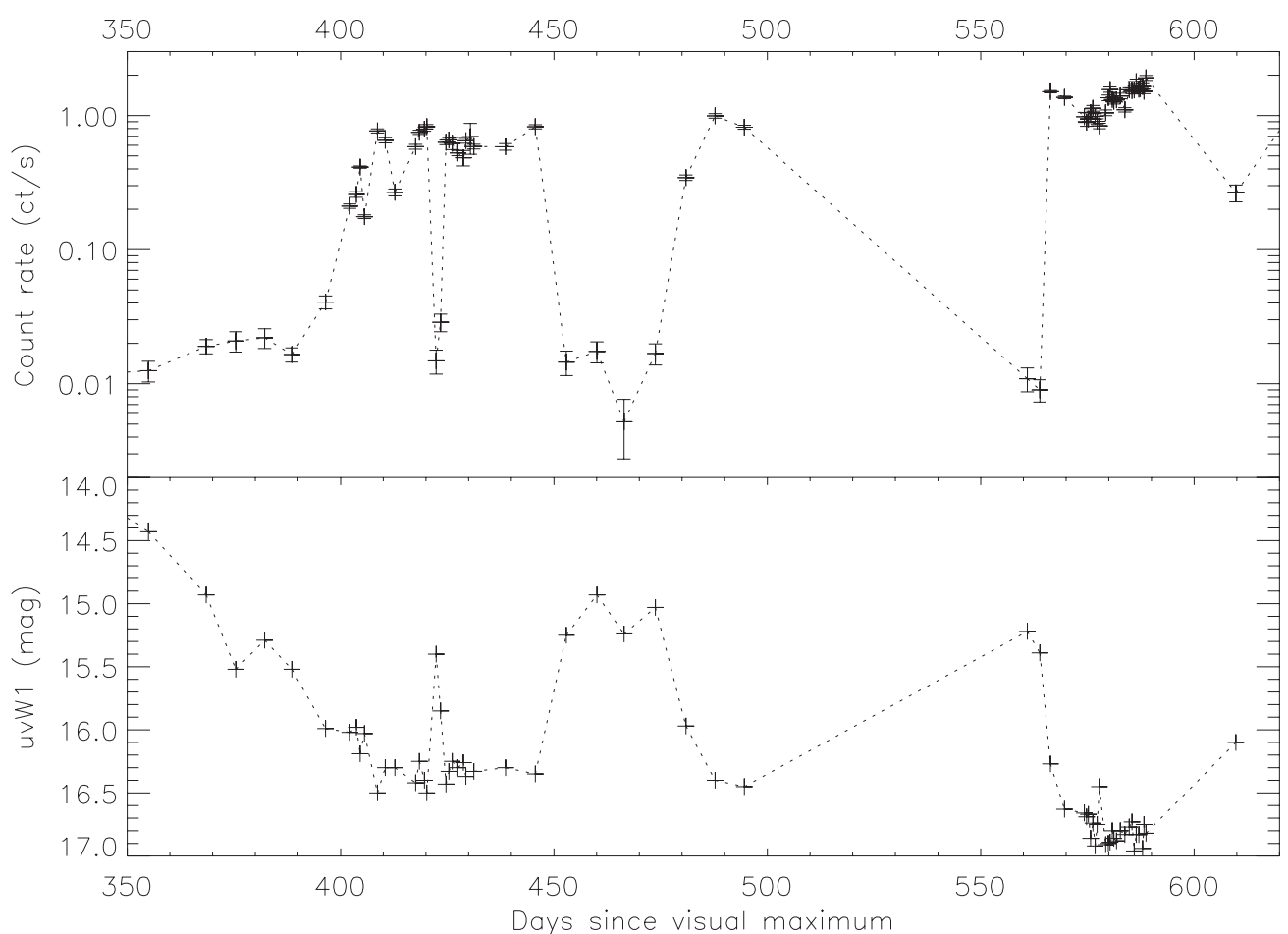

Figure 16. Swift X-ray (top panel) and uvw1 (bottom panel) light curve for V458 Vul. The $X$-axis is the number of days after visual maximum. Prior to day 400 , V458 Vul was in transition to X-ray maximum. After day 400 the majority of the X-ray observations had a count rate of $\sim 1 \mathrm{counts} \mathrm{s}^{-1}$. However, during the later phase there are three periods where the X-ray counts declined by about a factor of 100 . During these times the uvw1 $\left(\lambda_{c}=2600 \AA\right)$ photometric brightness increased by a magnitude.

become optically brighter from the irradiation of the accretion disk and secondary by the larger WD photosphere. The source remains X-ray faint until the WD photosphere shrinks back to its original size. Figure 16 shows similar behavior in the Swift $\mathrm{X}$-ray and UV light curves of V458 Vul compiled from the data in Table 2. The $100 \times$ decline in the X-ray light curve is matched by a 1.5 mag increase in the UV light curve. Figure 14 shows that similar X-ray and UV variations can be achieved by large declines in the effective temperature. For example, a decline from $700,000 \mathrm{~K}$ to $500,000 \mathrm{~K}$ produces a factor of 85 decline in the X-ray count rate and a 1.1 mag uvw1 band increase. If the underlying phenomenon in $\mathrm{V} 458 \mathrm{Vul}$ is the same 


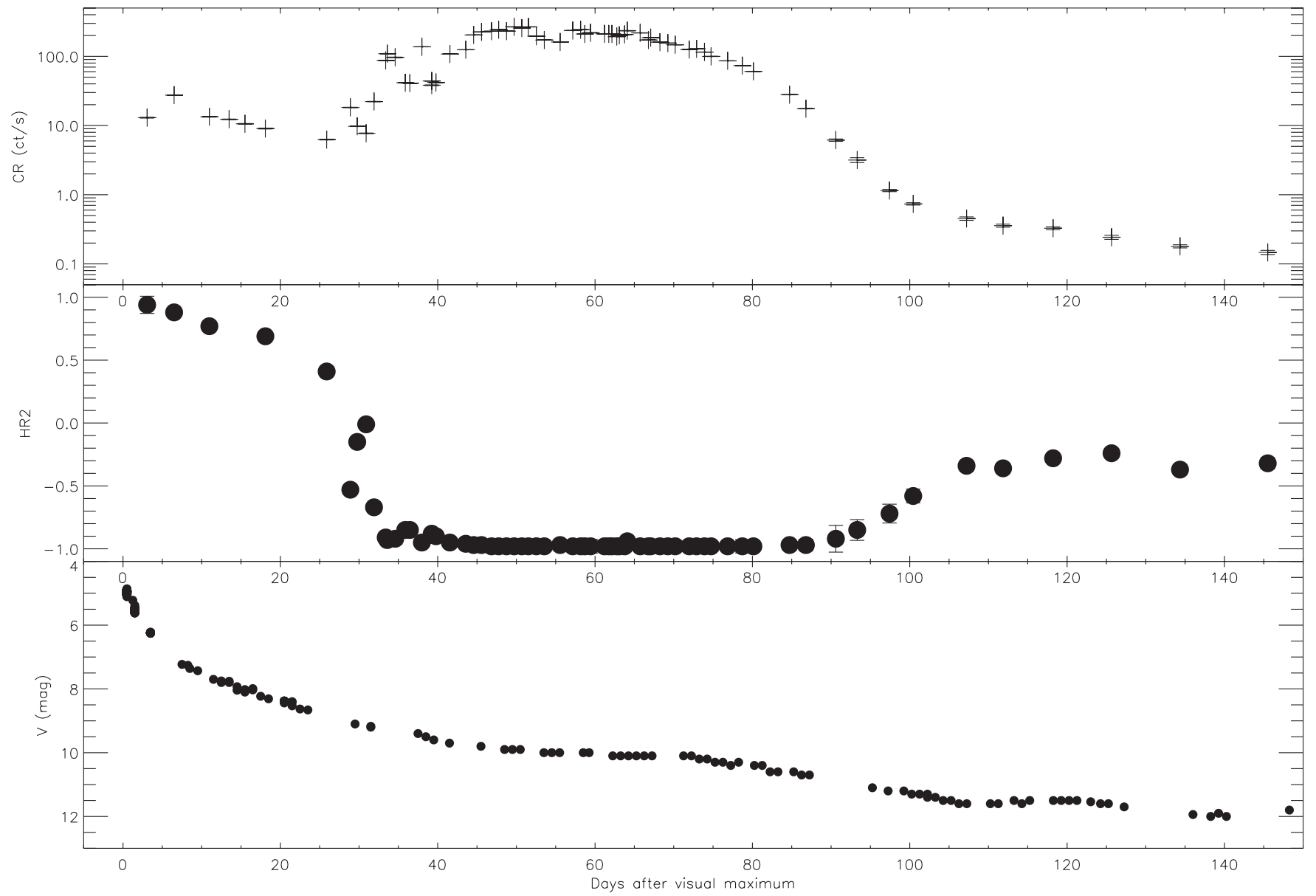

Figure 17. X-ray and optical evolution of RS Oph. The top panel is the Swift XRT $(0.3-10 \mathrm{keV})$ count rate and the middle panel is the hardness ratio, $(H-S) /(H+S)$ where $H=1-10 \mathrm{keV}$ and $S=0.3-1 \mathrm{keV}$. The bottom panel shows the AAVSO V-band light curve. Similar figures for U Sco, Nova LMC 2009 A, V407 Cyg, V2491 Cyg, KT Eri, and V2672 Oph are available in the online version.

(The complete figure set (seven images) is available in the online journal.)

as proposed for RX J0513.9-6951 (Reinsch et al. 2000) and Cal 83 (Greiner \& Di Stefano 2002) and the accretion disk has been reestablished, V458 Vul should have an orbital period of order one day to produce an accretion rate high enough to drive stable nuclear burning. However, Rodríguez-Gil et al. (2010) find a short orbital period of $\sim 98$ minutes implying that V458 Vul will not have a long-term SSS phase. ${ }^{14}$

\subsection{Estimating Timescales in a Variable Environment}

The variability of novae also raises questions about how confident one can be in the determination of turn-off times. A prime example can be seen in the X-ray light curve of V458 Vul in Figure 16. If monitoring had stopped following the four observations between days 450 and 480 the subsequent recovery would never have been found, and it would have been noted that V458 Vul had a turn-off time of 1.2 years instead of $\gtrsim 2.9$ years. While this could be a significant problem with the determination of a turn-off time in most novae, it is likely that the phenomenon observed in V458 Vul is rare. The X-ray behavior of V458 Vul, a $100 \times$ decline in flux and a subsequent recovery, is the only case observed in the 19 novae studied by Swift with SSS emission. Orio et al. (2001a) found no similar "reborn" SSSs in their review of the ROSAT all sky survey although some of the novae

\footnotetext{
${ }^{14}$ Note added in proof: Recent Swift observations of V458 Vul indicate that
} nuclear burning has ended with a turn-off timescale of $\sim 1300$ days. in M31 previously thought to be RNe with very rapid outburst timescales may actually be normal novae but with on/off states similar to those in V458 Vul. Since the sudden X-ray declines in V458 Vul also had corresponding UV rises, if these source exist in M31, they should be easily found with X-ray and UV capable facilities such as Swift and XMM.

\subsection{SSS in RNe and the Light Curve Plateau}

A plateau in visible light of $\mathrm{RNe}$ is speculated to arise from the reradiation of the SSS emission from an accretion disk dominating the emission after the free-free emission has faded (Hachisu et al. 2008). Once nuclear burning ends and the accretion disk is no longer irradiated, the light curve continues its decline to quiescence. Figures 17.1 and 17.2 show that the optical plateaus are nearly coincident with the SSS emission in RS Oph and U Sco.

The other well-observed RNe in the Swift archive are novae LMC 2009a and V407 Cyg. LMC 2009a was previously seen in outburst in 1971 (Liller 2009). It had a much longer SSS phase than RS Oph and U Sco and it ended 270 days after maximum. Unfortunately, the $V$-band light curve compiled from the AAVSO archives and our own SMARTS photometry does not extend beyond 110 days after visual maximum so we cannot determine whether an optical plateau was observed later in this outburst (see Figure 17.3). However, the Swift uvw2 and SMARTS $B$-band light curves are relatively flat during the SSS 
phase (see M. F. Bode et al. 2011, in preparation), indicating LMC 2009a did go through an optical plateau phase. The data are not as extensive for V407 Cyg but the rise in the soft $\mathrm{X}$-ray emission consistent with nuclear burning on the WD (see Section 4.3) is coincident with a short plateau in the optical light curve as shown in Figure 17.4.

There are three other novae with well-observed SSS phases in the Swift archive that are suspected to be RNe based on their outburst characteristics. The novae are V2491 Cyg, KT Eri, and V2672 Oph. Figure 17.5 shows that there is no indication of a plateau in V2491 Cyg while it was an SSS. However, the SSS phase in V2491 Cyg was extremely short, $<10$ days, which may not be sufficient time to produce a noticeable optical plateau or the system did not have its accretion disk reform this early in the outburst.

The early outburst spectra of KT Eri were indicative of the $\mathrm{He} / \mathrm{N}$ class with high expansion velocities typical of $\mathrm{RNe}$ (Ragan et al. 2009). KT Eri also had short X-ray light curve modulation similar to RS Oph, see Section 5.3.2 and Bode et al. (2010), while M. F. Bode et al. (2011, in preparation) draw attention to KT Eri's similarities with the X-ray behavior of LMC 2009a. The X-ray and $V$-band observations are shown in Figure 17.6. The AAVSO $V$-band light curve shows a flattening at 80 days after visual maximum or about 10 days after KT Eri became an SSS implying there was an optical plateau.

The case for V2672 Oph as a RNe is based on its extreme expansion velocities at maximum (Ayani et al. 2009) and early radio synchrotron emission similar to that observed in RS Oph (Krauss Hartman et al. 2009). Munari et al. (2011b) also find many similarities between V2672 Oph and U Sco. Unfortunately, the X-ray and optical observations were hampered due to the faintness at visual maximum and the relatively large column density. Based on the hardness ratio, V2672 Oph was in its SSS phase between days 15 and 30 after visual maximum (Figure 17.7). The AAVSO $V$-band light curve is supplemented with SMARTS $V$-band photometry, which shows a plateau between day 10 and 50 after visual maximum.

Of the four known RNe and three suspected RNe, there are sufficient optical data to reveal the presence of a plateau in six. Of those six all but V2491 Cyg have evidence of an optical plateau correlated with the X-ray SSS emission. However, Schaefer (2010) finds that not all Galactic RNe have optical plateaus. It is interesting to note that if the plateau phase is caused by reradiation off an accretion disk as suggested by Hachisu et al. (2008) then there is no effect on the presence or strength of the plateau due to the inclination of the system. One would expect the effect in more face-on systems such as RS Oph $\left(i=39_{-10^{+1}}^{\circ}\right.$; Ribeiro et al. 2009) than in edgeon systems such as U Sco $(i=82.7 \pm 2.9$; Thoroughgood et al. 2001). Regardless of the root cause of optical plateaus, their presence can clearly be used as a proxy signature of SSS emission. However, it should be stressed that while optical plateaus likely indicate soft X-ray emission, the start and ending of this phase in the optical light curve does not necessarily correspond to the turn-on and turn-off times in the SSS phase. Relationships between the optical timescales and the X-ray are only weakly correlated, e.g., Figure 9, and the two phases do not always align in the RN and suspected RN in this sample (Figures 17.1-17.7).

Optical/NIR plateaus should only be observed in RNe and other fast novae that eject very little mass. In slower novae the later spectra (i.e., several tens of weeks after maximum light) are dominated by hydrogen recombination and nebular line emission effectively hiding any irradiation effects. The continuum from the WD or a hot accretion disk can only be observed after the ejecta have sufficiently cleared.

\subsection{SSS Proxies at Other Wavelengths: The $[\mathrm{Fe} \mathrm{X}]$ Line}

Vanlandingham et al. (2001) used the evolution of UV emission line light curves developed in Shore et al. (1996) for V1974 Cyg to estimate turn-off times. This allowed Vanlandingham et al. (2001) to determine the nuclear burning timescales of five novae with no pointed X-ray observations but significant amounts of $I U E$ data. Unfortunately, it is currently difficult to obtain sufficient UV emission line data to utilize this technique while the optical plateau (Section 5.5) only applies to fast and $\mathrm{RNe}$. Another X-ray proxy is needed for slower novae.

The emergence of the coronal [Fe X] $6375 \AA$ line in the nebular spectra of novae has been long recognized as a strong indication of photoionization of the ejecta from a hot source (e.g., Krautter \& Williams 1989). With an ionization potential of $235 \mathrm{eV}$, an ejected shell must be highly ionized by a hot WD to produce [Fe $\mathrm{X}]$. While shocks can produce high temperatures, they only contribute very early in the outburst and are insignificant during the later nebular phase when $[\mathrm{Fe} \mathrm{x}]$ is typically observed, in all but the RS Oph-type RNe. For example, strong [Fe $\mathrm{x}]$ and [Fe XIV] $5303 \AA$ emission has been observed in RS Oph in all outbursts with adequate spectroscopic coverage (Ribeiro et al. 2009). However, these lines appear well before the SSS phase begins. A relationship between [Fe $\mathrm{X}]$ and soft X-ray emission has not been previously demonstrated but can be strengthened with our larger nova sample.

Seven novae with confirmed SSS emission, GQ Mus (Krautter \& Williams 1989), V1974 Cyg (Rafanelli et al. 1995), V1494 Aql (Iijima \& Esenoglu 2003), V723 Cas (Ness et al. 2008a), V574 Pup (Helton 2010), V597 Pup, and V1213 Cen (Schwarz et al. 2010) all had strong [Fe X] lines in their late nebular spectra. Example spectra of V597 Pup and V1213 Cen from our SMARTS archive are shown in Figure 18. In addition, extensive optical spectra from our Steward Observatory northern hemisphere nova monitoring campaign shows that V2467 Cyg may also have had weak [Fe X] emission at the same time it was an SSS but this cannot be confirmed due to nearby O I lines. These novae clearly show that the presences of strong $[\mathrm{Fe} \mathrm{X}]$ in the optical spectrum is indicative of underlying SSS emission. To our knowledge there has never been a nova with strong [Fe $\mathrm{x}]$ emission that was not also an SSS during contemporaneous X-ray observations. While additional optically and X-ray observations are needed to fully test this hypothesis, ground-based spectroscopic monitoring is a powerful tool for detecting SSS novae from [ $\mathrm{Fe} \mathrm{x}]$ emission in novae with significant ejected mass. The $\mathrm{RNe}$ and very fast $\mathrm{CNe}$ with rapid turn-on/off times are not strong photoionization sources long enough to produce $[\mathrm{Fe} \mathrm{X}]$ in their meager ejected shells.

\section{SUMMARY}

Over the last decade our knowledge of the X-ray behavior of novae has increased dramatically with the launch of the latest generation X-ray facilities. Observations of novae when they are radiating the majority of their flux in the soft X-ray band provide critical insight into the behavior of the WD and TNR processes. Currently, 26 Galactic/Magellanic novae have been observed as SSSs of which 19 such classifications have come from over $2 \mathrm{Ms}$ of Swift observations during the last five years. 

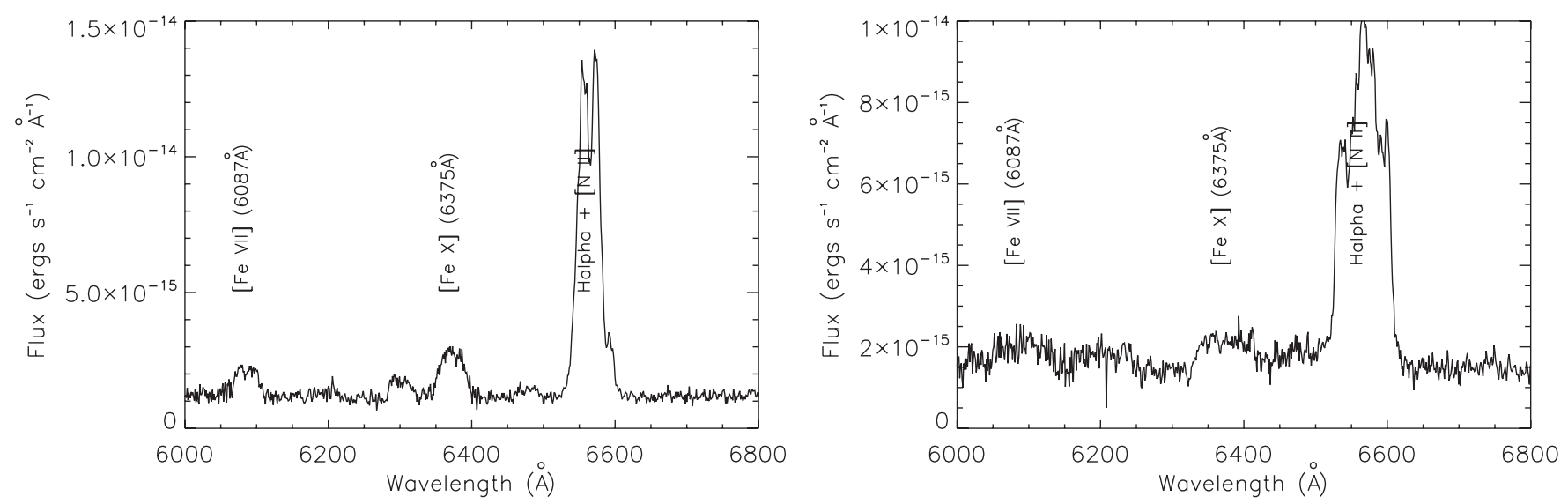

Figure 18. [Fe x] $6375 \AA$ A emission in V1213 Cen (left) and V597 Pup (right) obtained on 2010 June 27th (415 days from visual maximum) and 2008 March 26 th (133 days from visual maximum), respectively. The [Fe VII] $6087 \AA$ line is also visible in both spectra. Strong [Fe x] emission relative to [Fe VII] is a hallmark of novae in their SSS phase.

This large sample shows that individual novae can differ significantly from fits to smaller ensemble data sets such as the $t_{2}$ relationship of Hachisu \& Kato (2010) and the expansion velocity relationship of Greiner et al. (2003). Surprisingly, there is also no relationship between orbital period and the duration of nuclear burning. This large data set confirms that many factors are in play in the evolution of the SSS phase.

The duration of nuclear burning on the WD is short, with $89 \%$ of the novae have turned off within three years in this expanded sample. The median duration of the sample is 1.4 years. This contrasts with the same distribution in M31, which is peaked at longer burning novae. The difference is likely a selection effect between the two surveys.

The new Swift data are also challenging our understanding of novae with highly variable X-ray light curves both during the rise to and at X-ray maximum. Various mechanisms are likely at work to produce the variability. Additional observations are warranted not only to help decipher the current peculiar observations but also to be sure that we have captured the full range of variability behaviors both periodic and nonperiodic that novae may yet produce. Long XMM and Chandra grating observations can explore the short-term oscillations more effectively than Swift, whereas Swift can easily track the long-term behavior such as turn-on and turn-off times. In addition, simultaneous X-ray/UV observations only available through $X M M$ and Swift will continue to be a powerful tool to test the evolution of the emission from the WD during the outburst.

To date no strong dust-forming novae have been detected as an SSS. V2362 Cyg did have detectable soft X-ray photons but it was not similar to any of the other SSS novae. While V574 Pup and V2467 Cyg were in the SSS phase they had IR features indicating weak silicate dust emission. V1280 Sco had a large DQ Her-like dust event but also ejected so much material and at a low velocity that it is still optically thick several years after visual maximum. Any SSS phase will not be detected until this material clears.

There are optical behaviors that track SSS emission in novae. For the RNe with well-defined plateaus in their optical light curves, RS Oph and U Sco, the X-ray light curves reach maximum around the same time. However, not all RNe and suspected RNe in the sample had optical plateaus even though they had well-documented observations during X-ray maximum. An optical spectroscopic signature indicative of an
SSS phase is the presence of strong [Fe x] $6375 \AA$ emission. In the sample, all novae with [Fe $\mathrm{X}]$ that were subsequently observed in the X-ray were SSSs. These were slower novae that ejected significantly more material than the RNe. The inverse of the $[\mathrm{Fe} x]$ relationship does not hold since the source may turn off before [Fe $\mathrm{X}]$ can be created in the ejecta. While the presences of neither optical plateaus or [Fex] has yet been shown to be simultaneous with SSS emission, these relationships offer excellent opportunities to use ground-based monitoring to coordinate X-ray observations during the important SSS phase.

Additional X-ray data need to be collected since the sample is statistically meager with only 26 known SSS novae and is smaller still for novae with early, hard X-ray detections. Trends can be difficult to confirm given the wide range of behavior observed during the different X-ray phases. With the sample heavily biased toward fast and RNe, efforts should be expended on novae that are not currently well represented in the X-ray sample such as slow and dust-forming novae. The monitoring of the two slow novae that have been detected as X-ray sources, V5558 Sgr and V1280 Sco, but have not yet evolved to an SSS state, will help in understanding slow systems. Likewise, Swift monitoring of the two long-lasting SSSs, V723 Cas and V458 Vul, are also of interest since they are rare, and thus, important to our understanding of why they persist.

Finally, it is important to continue to collect X-ray observations of novae and build on this sample. This analysis shows that each nova is in some ways unique and that attempts to predict their behavior based on a relationship to a single observational value, e.g., $t_{2}$ versus the nuclear burning timescale, is fraught with difficulties. Some of these problems can be addressed by expanding the sample to include regions of the parameter space that are not well represented. This X-ray sample includes few slow novae which likely explains the differences between the nuclear burning timescale of the Milky Way and M31 surveys. It is also equally important to obtain numerous, high-quality data for all bright novae through their evolution and at different wavelengths from X-ray to radio. Multiwavelength observations are critical to properly interpret nova phenomena such as the apparent early turn-off in V458 Vul and to verify periodicities seen in the X-ray, particularly potential orbital periods. With the understanding that comes from a few well-observed novae like RS Oph and U Sco, the entire nova data set can be anchored to nova theory. These large data sets also reveal new phenomena such as the strong X-ray variability that is not 
appreciated in novae with sparser observations or detected at other wavelengths.

This research has made use of data obtained from NASA's Swift satellite. We thank Neil Gehrels and the Swift team for generous allotments of $\mathrm{ToO}$ and fill in time. Funding support from NASA NNH08ZDA001N1. Stony Brook University's initial participation in the SMARTS consortium was made possible by generous contributions from the Dean of Arts and Sciences, the Provost, and the Vice President for Research of Stony Brook University. We acknowledge with thanks the variable star observations from the AAVSO International Database contributed by observers worldwide and used in this research. J.P.O., K.P., P.E. and A.B. acknowledge the support of the STFC. S.S. acknowledges partial support from NASA and NSF grants to ASU. J.J.D. was supported by NASA contract NAS8-39073 to the Chandra $\mathrm{X}$-ray Center.

Facilities: Swift(UVOT/XRT), AAVSO, CTIO:1.3m, CTIO:1.5m, Bok(B\&C spectrograph), Spitzer(IRS)

Note added in proof: Recent Swift observations of V458 Vul indicate that nuclear burning has ended with a turn-off timescale of $\sim 1300$ days.

\section{REFERENCES}

Abdo, A. A., Ackermann, M., Ajello, M., et al. 2010, Science, 329, 817 Ak, T., Retter, A., \& Liu, A. 2005, PASA, 22, 298

Anupama, G. C. 2010, ATel, 2411, 1

Anupama, G. C., \& Dewangan, G. C. 2000, AJ, 119, 1359

Austin, S. J., Wagner, R. M., Starrfield, S., et al. 1996, AJ, 111, 869

Ayani, K., Kawabata, T., \& Liller, W. 2001, IAU Circ., 7589, 2

Ayani, K., \& Murakami, N. 2008, IAU Circ., 8948, 2

Ayani, K., Murakami, N., Hata, K., et al. 2009, IAU Circ., 9064, 2

Baklanov, A., Pavlenko, E., \& Berezina, E. 2008, ATel, 1514, 1

Balman, S., Krautter, J., \& Oegelman, H. 1998, ApJ, 499, 395

Balman, S., Nasiroglu, I., \& Akyuz, A. 2009, ATel, 2137, 1

Balman, Ş., Retter, A., \& Bos, M. 2006, AJ, 131, 2628

Beardmore, A. P., Balman, S., Osborne, J. P., et al. 2010a, ATel, 2423, 1

Beardmore, A. P., Osborne, J. P., Page, K. L., et al. 2010b, Astron. Nachr., 331, 156

Bode, M. F. 2010, Astron. Nachr., 331, 160

Bode, M. F., Harman, D. J., O’Brien, T. J., et al. 2007, ApJ, 665, L63

Bode, M. F., O’Brien, T. J., Osborne, J. P., et al. 2006, ApJ, 652, 629

Bode, M. F., Osborne, J. P., Page, K. L., et al. 2008, in ASP Conf. Ser. 401, RS Ophiuchi (2006) and the Recurrent Nova Phenomenon, ed. A. Evans et al. (San Francisco, CA: ASP), 269

Bode, M. F., Osborne, J. P., Page, K. L., et al. 2009a, ATel, 2001, 1

Bode, M. F., Osborne, J. P., Page, K. L., et al. 2009b, ATel, 2025, 1

Bode, M. F., Osborne, J. P., Page, K. L., et al. 2010, ATel, 2392, 1

Bohlin, R. C., Savage, B. D., \& Drake, J. F. 1978, ApJ, 224, 132

Bos, M., Retter, A., Cook, L., \& Novak, R. 2001, IAU Circ., 7665, 2

Boyd, D., \& Poyner, G. 2006, J. Br. Astron. Assoc., 116, 320

Bruch, A. 2001, IBVS, 5138, 1

Buil, C., \& Fujii, M. 2007, IAU Circ., 8862, 2

Burlak, M. A. 2008, Astron. Lett., 34, 249

Burrows, D. N., Hill, J. E., Nousek, J. A., et al. 2005, Space Sci. Rev., 120, 165

Camilleri, P., McNaught, R. H., Gilmore, A. C., \& Kilmartin, P. M. 1992, IAU Circ., 5422, 1

Capaccioli, M., della Valle, M., Rosino, L., \& D’Onofrio, M. 1989, AJ, 97, 1622 Chesneau, O., Banerjee, D. P. K., Millour, F., et al. 2008, A\&A, 487, 223

Chochol, D., Grygar, J., Pribulla, T., et al. 1997, A\&A, 318, 908

Chochol, D., \& Pribulla, T. 1998, Contrib. Astron. Obs. Skalnate Pleso, 28, 121

Cragg, T., Charles, P., Nikoloff, I., et al. 1983, IAU Circ., 3766, 1

Csák, B., Kiss, L. L., Retter, A., Jacob, A., \& Kaspi, S. 2005, A\&A, 429, 599

Czart, K., Lewandowski, M., Maciejewski, G., et al. 2006, ATel, 792, 1

Darnley, M. J., Ribeiro, V. A. R. M., Bode, M. F., \& Munari, U. 2011, A\&A, 530, A70

Das, R. K., Banerjee, D. P. K., Ashok, N. M., \& Chesneau, O. 2008, MNRAS, 391, 1874

De Young, J. A., \& Schmidt, R. E. 1994, ApJ, 431, L47

della Valle, M., \& Livio, M. 1995, ApJ, 452, 704 della Valle, M., Pasquini, L., Daou, D., \& Williams, R. E. 2002, A\&A, 390, 155 della Valle, M., Pizzella, A., Bernardi, M., et al. 1998b, IAU Circ., 6848, 1 Diaz, M. P., \& Steiner, J. E. 1989, ApJ, 339, L41

Diaz, M. P., Williams, R. E., Luna, G. J., Moraes, M., \& Takeda, L. 2010, AJ, 140,1860

Dickey, J. M., \& Lockman, F. J. 1990, ARA\&A, 28, 215

Dobrotka, A., \& Ness, J.-U. 2010, MNRAS, 405, 2668

Dobrotka, A., Retter, A., \& Liu, A. 2006, MNRAS, 371, 459

Dobrotka, A., Retter, A., \& Liu, A. 2008, A\&A, 478, 815

Drake, J. J., Laming, J. M., Ness, J.-U., et al. 2009, ApJ, 691, 418

Drake, J. J., \& Orlando, S. 2010, ApJ, 720, L195

Drake, J. J., Wagner, R. M., Starrfield, S., et al. 2003, ApJ, 584, 448

Duerbeck, H. W., Baptista, R., Dutra, C. M., \& Sterken, C. 2002, IAU Circ., 7799, 3

Duerbeck, H. W., \& Pompei, E. 2000, IAU Circ., 7457, 1

Ederoclite, A., Mason, E., Della Valle, M., et al. 2006, A\&A, 459, 875

Evans, A., Krautter, J., Vanzi, L., \& Starrfield, S. 2001, A\&A, 378, 132

Evans, A., \& Rawlings, J. M. 2008, in Classical Novae, ed. M. Bode \& A. Evans

(2nd ed.; Cambridge: Cambridge Univ. Press), 308

Evans, A., Woodward, C. E., Helton, L. A., et al. 2007a, ApJ, 671, L157

Evans, P. A., Beardmore, A. P., Page, K. L., et al. 2007b, A\&A, 469, 379

Evans, P. A., Beardmore, A. P., Page, K. L., et al. 2009, MNRAS, 397, 1177

Eyres, S. P. S., O’Brien, T. J., Beswick, R., et al. 2009, MNRAS, 395, 1533

Ferri, C., Hernanz, M., \& Sala, G. 2007, in ASP Conf. Ser. 372, 15th European

Workshop on White Dwarfs, ed. R. Napiwotzki \& M. R. Burleigh (San Francisco, CA: ASP), 519

Fujii, M. 1999, IAU Circ., 7324, 1

Gallagher, J. S., III, \& Code, A. D. 1974, ApJ, 189, 303

Gallagher, J. S., \& Starrfield, S. 1978, ARA\&A, 16, 171

Gehrz, R. D., Truran, J. W., Williams, R. E., \& Starrfield, S. 1998, PASP, 110, 3

Gilmore, A. C., \& Kilmartin, P. M. 2005, IAU Circ., 8559, 2

Goranskij, V. P., Katysheva, N. A., Kusakin, A. V., et al. 2007, Astrophys. Bull., 62,125

Greiner, J., \& Di Stefano, R. 2002, A\&A, 387, 94

Greiner, J., Orio, M., \& Schartel, N. 2003, A\&A, 405, 703

Hachisu, I., \& Kato, M. 2007, ApJ, 662, 552

Hachisu, I., \& Kato, M. 2010, ApJ, 709, 680

Hachisu, I., Kato, M., Kiyota, S., et al. 2008, in ASP Conf. Ser. 401, RS Ophiuchi (2006) and the Recurrent Nova Phenomenon, ed. A. Evans et al. (San Francisco, CA: ASP), 206

Hanzl, D., Reszelski, M., Hornoch, K., \& Lehky, M. 2000, IAU Circ., 7411, 3 Helton, L. A. 2010, PhD thesis, Univ. Minnesota

Helton, L. A., Woodward, C. E., Vanlandingham, K., \& Schwarz, G. J. 2008a, CBET, 1379, 1

Helton, L. A., Woodward, C. E., Vanlandingham, K., \& Schwarz, G. J. 2008b, CBET, 1448, 1

Helton, L. A., Woodward, C. E., Walter, F. M., et al. 2010, AJ, 140, 1347

Henze, M., Pietsch, W., Haberl, F., et al. 2010, A\&A, 523, A89

Hernanz, M. 2008, in Classical Novae, ed. M. Bode \& A. Evans (2nd ed.; Cambridge: Cambridge Univ. Press), 252

Hernanz, M., Ferri, C., \& Sala, G. 2007, ATel, 1226, 1

Hernanz, M., \& José, J. 2005, Nucl. Phys. A, 758, 721

Hernanz, M., \& Sala, G. 2007, ApJ, 664, 467

Hernanz, M., \& Sala, G. 2010, Astron. Nachr., 331, 169

Hirosawa, K. 2010, CBET, 2199, 1

Hounsell, R., Bode, M. F., Hick, P. P., et al. 2010, ApJ, 724, 480

Iijima, T., Correia, A. P., Hornoch, K., \& Carvajal, J. 2007, CBET, 1006, 1

Iijima, T., \& Esenoglu, H. H. 2003, A\&A, 404, 997

Iijima, T., \& Rosino, L. 1996, IAU Circ., 6365, 2

José, J., Hernanz, M., \& Iliadis, C. 2006, Nucl. Phys. A, 777, 550

Kahabka, P., \& van den Heuvel, E. P. J. 1997, ARA\&A, 35, 69

Kalberla, P. M. W., Burton, W. B., Hartmann, D., et al. 2005, A\&A, 440, 775

Kang, T. W., Retter, A., Liu, A., \& Richards, M. 2006, AJ, 132, 608

Kato, T., \& Fujii, M. 2003, IAU Circ., 8132, 2

Kato, T., Fujii, M., \& Ayani, K. 2002b, IAU Circ., 7975, 2

Kato, T., Nishimura, H., Takamizawa, K., et al. 2001, IAU Circ., 7590, 2

Kato, T., Yamaoka, H., \& Liller, W. 2002a, IAU Circ., 7791, 2

Kimeswenger, S., Dalnodar, S., Knapp, A., et al. 2008, A\&A, 479, L51

Kiss, L., \& Derekas, A. 2005, IAU Circ., 8501, 2

Kiss, L. L., \& Thomson, J. R. 2000, A\&A, 355, L9

Kraft, R. P., Burrows, D. N., \& Nousek, J. A. 1991, ApJ, 374, 344

Krauss Hartman, M. I., Rupen, M. P., \& Mioduszewski, A. J. 2009, ATel, 2195, 1

Krautter, J. 2002, in AIP Conf. Proc. 637, Classical Nova Explosions, ed. M. Hernanz \& J. José (Melville, NY: AIP), 345

Krautter, J., Beuermann, K., Leitherer, C., et al. 1984, A\&A, 137, 307 
Krautter, J., Oegelman, H., Starrfield, S., Wichmann, R., \& Pfeffermann, E. 1996, ApJ, 456, 788

Krautter, J., \& Williams, R. E. 1989, ApJ, 341, 968

Lane, B. F., Retter, A., Eisner, J. A., et al. 2007, ApJ, 669, 1150

Lee, S., Pearce, A., Gilmore, C., et al. 1999, IAU Circ., 7176, 1

Liller, W. 1995, IAU Circ., 6143, 2

Liller, W. 2003, IAU Circ., 8126, 2

Liller, W. 2005, IAU Circ., 8559, 1

Liller, W. 2009, IAU Circ., 9019, 1

Liller, W., Allen, B., \& Pearce, A. 2005a, IAU Circ., 8635, 1

Liller, W., Heathcote, B., di Scala, G., \& Allen, W. 2007a, J. Am. Assoc. Var. Star Obs., 35, 359

Liller, W., Heathcote, B., Schmeer, P., et al. 2007b, IAU Circ., 8800, 1

Liller, W., Jacques, C., Pimentel, E., Aguiar, J. G. D. S., \& Shida, R. Y. 2005b, IAU Circ., 8596, 1

Liller, W., Monard, L. A. G., \& Africa, S. 2005c, IAU Circ., 8582, 2

Liller, W., Monard, L. A. G., Africa, S., Samus, N. N., \& Kazarovets, E. 2005d, IAU Circ., 8497, 1

Liller, W., Shida, R. Y., \& Jones, A. F. 2004, IBVS, 5582,

Liller, W., \& Stubbings, R. 2000, IAU Circ., 7453, 1

Liller, W., Tabur, V., Williams, P., et al. 2008, IAU Circ., 8990, 2

Lipkin, Y., Leibowitz, E. M., Retter, A., \& Shemmer, O. 2001, MNRAS, 328, 1169

Lipkin, Y. M., \& Leibowitz, E. M. 2008, MNRAS, 387, 289

Livio, M., \& Truran, J. W. 1994, ApJ, 425, 797

Lloyd, H. M., O’Brien, T. J., Bode, M. F., et al. 1992, Nature, 356, 222

Lu, G., Zhu, C., Wang, Z., Huo, W., \& Yang, Y. 2011, MNRAS, 413, L11

Lyke, J. E., \& Campbell, R. D. 2009, AJ, 138, 1090

Lynch, D. K., Rudy, R. J., Mazuk, S., \& Puetter, R. C. 2000, ApJ, 541, 791

Lynch, D. K., Rudy, R. J., Mazuk, S., et al. 2004, IAU Circ., 8368, 3

Lynch, D. K., Rudy, R. J., Venturini, C. C., et al. 2006a, IAU Circ., 8730, 5

Lynch, D. K., Russell, R. W., Rudy, R. J., \& Woodward, C. E. 2007, IAU Circ., 8883,1

Lynch, D. K., Russell, R. W., Rudy, R. J., \& Woodward, C. E. 2009, BAAS, 41, 471

Lynch, D. K., Woodward, C. E., Geballe, T. R., et al. 2006b, ApJ, 638, 987

Lynch, D. K., Woodward, C. E., Gehrz, R., et al. 2008, AJ, 136, 1815

MacDonald, J., Fujimoto, M. Y., \& Truran, J. W. 1985, ApJ, 294, 263

Mason, E., Brandeker, A., Ederoclite, A., Della Valle, M., \& Liller, W. 2005, IAU Circ., 8582, 3

Mazuk, S., Lynch, D. K., Rudy, R. J., et al. 2005, IAU Circ., 8644, 1

Mazuk, S., Lynch, D. K., Rudy, R. J., et al. 2007, IAU Circ., 8848, 1

Meng, X., \& Yang, W. 2010, Ap\&SS, 329, 287

Morgan, G. E., Ringwald, F. A., \& Prigge, J. W. 2003, MNRAS, 344, 521

Munari, U., Goranskij, V. P., Popova, A. A., et al. 1996, A\&A, 315, 166

Munari, U., Henden, A., Pojmanski, G., et al. 2006a, MNRAS, 369, 1755

Munari, U., Henden, A., Valentini, M., et al. 2008a, MNRAS, 387, 344

Munari, U., Henden, A., Valisa, P., Dallaporta, S., \& Righetti, G. L. 2010, PASP, 122,898

Munari, U., Joshi, V. H., Ashok, N. M., et al. 2011a, MNRAS, 410, L52

Munari, U., Margoni, R., \& Stagni, R. 1990, MNRAS, 242, 653

Munari, U., Moretti, S., \& Tomaselli, S. 2007a, CBET, 1029, 1

Munari, U., Orio, M., Valentini, M., et al. 2007b, CBET, 1010, 1

Munari, U., Ribeiro, V. A. R. M., Bode, M. F., \& Saguner, T. 2011b, MNRAS, 410,525

Munari, U., Siviero, A., Moretti, S., et al. 2008b, CBET, 1352, 1

Munari, U., Siviero, A., Navasardyan, H., \& Dallaporta, S. 2006b, A\&A, 452, 567

Munari, U., Siviero, A., Valisa, P., et al. 2007c, CBET, 1183, 1

Munari, U., Valisa, P., Dalla Via, G., \& Dallaporta, S. 2007d, CBET, 852, 1

Munari, U., \& Zwitter, T. 1997, A\&A, 318, 269

Naito, H., \& Fujii, M. 2008, IAU Circ., 8972, 2

Naito, H., Narusawa, S., Yamaoka, H., \& Fujita, Y. 2007, IAU Circ., 8812, 2

Naito, H., \& Tokimasa, N. 2007, IAU Circ., 8896, 2

Naito, H., Tokimasa, N., \& Yamaoka, H. 2005a, IAU Circ., 8484, 1

Naito, H., Tokimasa, N., Yamaoka, H., \& Fujii, M. 2005b, IAU Circ., 8576, 2

Nakano, S., Beize, J., Jin, Z.-W., et al. 2008a, IAU Circ., 8934, 1

Nakano, S., Kaneda, H., \& Kadota, K. 2008b, IAU Circ., 8927, 2

Nakano, S., Kushida, Y., Aoki, M., \& Garradd, G. J. 1998, IAU Circ., 6847, 2

Nakano, S., Nishimura, H., Kadota, K., et al. 2007a, IAU Circ., 8824, 1

Nakano, S., Nishimura, H., Wakuda, S., \& Kadota, K. 2005, IAU Circ., 8483, 1

Nakano, S., Nishiyama, K., Kabashima, F., \& Kadota, K. 2008c, IAU Circ. 8948, 3

Nakano, S., Nishiyama, K., Kabashima, F., et al. 2008d, IAU Circ., 8950, 1

Nakano, S., Nishiyama, K., Kabashima, F., et al. 2008e, IAU Circ., 8972, 1

Nakano, S., Tago, A., Itagaki, K., \& Kadota, K. 2007b, CBET, 1181, 1
Nakano, S., Tago, A., Nishiyama, K., \& Sakamoto, T. 2007c, IAU Circ., 8821 1

Nakano, S., Takamizawa, K., Kushida, Y., \& Kato, T. 1998, IAU Circ., 6941, 1 Nakano, S., Yamaoka, H., Itagaki, K., et al. 2009, IAU Circ., 9064, 1

Nelson, T., \& Orio, M. 2007, ATel, 1202, 1

Nelson, T., \& Orio, M. 2009, ATel, 1910, 1

Nelson, T., Orio, M., Cassinelli, J. P., et al. 2008, ApJ, 673, 1067

Ness, J.-U., Drake, J. J., Beardmore, A. P., et al. 2009a, AJ, 137, 4160

Ness, J.-U., Drake, J. J., Starrfield, S., et al. 2010a, ATel, 2418, 1

Ness, J.-U., Drake, J. J., Starrfield, S., et al. 2010b, ATel, 2469, 1

Ness, J.-U., Osborne, J. P., Dobrotka, A., et al. 2011, ApJ, 733, 70

Ness, J.-U., Schwarz, G., Starrfield, S., et al. 2008a, AJ, 135, 1328

Ness, J.-U., Schwarz, G., Woodward, C. E., et al. 2009b, ATel, 2063, 1

Ness, J.-U., Schwarz, G. J., Retter, A., et al. 2007, ApJ, 663, 505

Ness, J.-U., Starrfield, S., Burwitz, V., et al. 2003, ApJ, 594, L127

Ness, J.-U., Starrfield, S., Gonzalez, R., et al. 2008b, ATel, 1573, 1

Ness, J.-U., Starrfield, S., Gonzalez, R., et al. 2008c, ATel, 1561, 1

Ness, J.-U., Starrfield, S., Schwarz, G. J., et al. 2008d, IAU Circ., 8911, 2

Nishimura, H., Nakano, S., Liller, W., et al. 2004, IAU Circ., 8306, 1

Nishiyama, K., Kabashima, F., Pojmanski, G., et al. 2009, IAU Circ., 9061, 1

O’Brien, T. J., \& Bode, M. F. 2008, in Classical Novae, ed. M. Bode \& A. Evans (2nd ed.; Cambridge: Cambridge Univ. Press), 285

O’Brien, T. J., Bode, M. F., \& Kahn, F. D. 1992, MNRAS, 255, 683

O’Brien, T. J., Lloyd, H. M., \& Bode, M. F. 1994, MNRAS, 271, 155

Orio, M., Balman, S., della Valle, M., Gallagher, J., \& Ögelman, H. 1996, ApJ, 466,410

Orio, M., Covington, J., \& Ögelman, H. 2001a, A\&A, 373, 542

Orio, M., Nelson, T., Luna, J., et al. 2010, ATel, 2451, 1

Orio, M., Parmar, A., Benjamin, R., et al. 2001b, MNRAS, 326, L13

Orio, M., Tepedelenlioglu, E., Starrfield, S., Woodward, C. E., \& Della Valle, M. 2005, ApJ, 620, 938

Osborne, J., Page, K., Beardmore, A., et al. 2006, ATel, 770, 1

Osborne, J. P., Page, K. L., Beardmore, A. P., et al. 2011, ApJ, 727, 124

Osborne, J. P., Page, K. L., Wynn, G., et al. 2010, ATel, 2442, 1

Page, K. L., Osborne, J. P., Evans, P. A., et al. 2010, MNRAS, 401, 121

Page, K. L., Osborne, J. P., Read, A. M., et al. 2009, A\&A, 507, 923

Pagnotta, A., Schaefer, B. E., Xiao, L., Collazzi, A. C., \& Kroll, P. 2009, AJ, 138,1230

Pereira, A. J. S., McGaha, J. E., Young, J., \& Rhoades, H. 2007, IAU Circ. 8895, 1

Perry, R. B., Venturini, C. C., Rudy, R. J., et al. 2005, IAU Circ., 8638, 1

Pietsch, W. 2010, Astron. Nachr., 331, 187

Pietsch, W., Haberl, F., Sala, G., et al. 2007, A\&A, 465, 375

Pigulski, A. 2009, IAU Circ., 9043, 2

Poggiani, R. 2009, Astron. Nachr., 330, 77

Poggiani, R. 2008a, Ap\&SS, 315, 79

Poggiani, R. 2008b, New Astron., 13, 557

Poggiani, R. 2010, New Astron., 15, 170

Pojmanski, G., Nakano, S., Nishimura, H., Hashimoto, N., \& Urata, T. 2005a, IAU Circ., 8574, 1

Pojmanski, G., \& Oksanen, A. 2005, IAU Circ., 8540, 1

Pojmanski, G., Szczygiel, D., Pilecki, B., et al. 2009, IAU Circ., 9043, 1

Pojmanski, G., Yamaoka, H., Haseda, K., et al. 2005b, IAU Circ., 8617, 1

Pojmanski, G., Yamaoka, H., Kiyota, S., et al. 2006, IAU Circ., 8671, 1

Poole, T. S., Breeveld, A. A., Page, M. J., et al. 2008, MNRAS, 383, 627

Prieto, J. L., Denney, K., Pejcha, O., \& Wagner, R. M. 2008, ATel, 1835, 1

Puetter, R. C., Rudy, R. J., Lynch, D. K., et al. 2005, IAU Circ., 8640, 2

Rafanelli, P., Rosino, L., \& Radovich, M. 1995, A\&A, 294, 488

Ragan, E., Brozek, T., Suchomska, K., et al. 2009, ATel, 2327, 1

Rauch, T., Orio, M., Gonzales-Riestra, R., et al. 2010, ApJ, 717, 363

Read, A. M., Saxton, R. D., Jonker, P. G., et al. 2009, A\&A, 506, 1309

Read, A. M., Saxton, R. D., Torres, M. A. P., et al. 2008, A\&A, 482, L1

Reinsch, K., van Teeseling, A., King, A. R., \& Beuermann, K. 2000, A\&A, 354 L37

Retter, A., O’Toole, S., Stathakis, R., Pogson, J., \& Naylor, T. 2002, IAU Circ., 7809, 1

Ribeiro, V. A. R. M., Bode, M. F., Darnley, M. J., et al. 2009, ApJ, 703, 1955

Rodríguez-Gil, P., Santander-García, M., Knigge, C., et al. 2010, MNRAS, 407, L21

Roming, P. W. A., Kennedy, T. E., Mason, K. O., et al. 2005, Space Sci. Rev., 120,95

Rudy, R. J., Lynch, D. K., Russell, R. W., Gilbert, A. M., \& Woodward, C. E. 2008a, IAU Circ., 8957, 1

Rudy, R. J., Lynch, D. K., Russell, R. W., \& Woodward, C. E. 2007, IAU Circ., 8884, 2

Rudy, R. J., Lynch, D. K., Russell, R. W., Woodward, C. E., \& Covey, K. 2008b, IAU Circ., 8938, 2 
Rudy, R. J., Lynch, D. K., Russell, R. W., et al. 2009, American Astronomical Society Meeting Abstract, 214, 2144, \#428.06

Rudy, R. J., Rossano, G., \& Puetter, R. 1989, ApJ, 383, 344

Rudy, R. J., Russell, R. W., Lynch, D. K., \& Widths, I. 2005, IAU Circ., 8523, 4

Rudy, R. J., Russell, R. W., Lynch, D. K., \& Woodward, C. E. 2008c, IAU Circ., 8936, 2

Rudy, R. J., Tessensohn, T. K., Mazuk, S., et al. 1998, IAU Circ., 7049, 1

Russell, R. W., Lynch, D. K., \& Rudy, R. J. 2005a, IAU Circ., 8579, 3

Russell, R. W., Lynch, D. K., Rudy, R. J., \& Woodward, C. E. 2008a, IAU Circ., 8936, 3

Russell, R. W., Rudy, R. J., Lynch, D. K., Gilbert, A. M., \& Woodward, C. E. 2008b, IAU Circ., 8956, 1

Russell, R. W., Rudy, R. J., Lynch, D. K., \& Golisch, W. 2005b, IAU Circ., 8524,2

Russell, R. W., Rudy, R. J., Lynch, D. K., \& Woodward, C. E. 2006, IAU Circ., 8710,2

Russell, R. W., Rudy, R. J., Lynch, D. K., et al. 2007, IAU Circ., 8846, 2

Russell, R. W., Rudy, R. J., Lynch, D. K., et al. 2008c, IAU Circ., 8948, 1

Sadakane, K., Tajitsu, A., Mizoguchi, S., Arai, A., \& Naito, H. 2010, PASJ, 62, L5

Saizar, P., Pachoulakis, I., Shore, S. N., et al. 1996, MNRAS, 279, 280

Sala, G., Hernanz, M., Ferri, C., \& Greiner, J. 2008, ApJ, 675, L93

Samus, N. N., \& Kazarovets, E. 2005, IAU Circ., 8502, 1

Schaefer, B. E. 2010, ApJS, 187, 275

Schaefer, B. E., \& Collazzi, A. C. 2010, AJ, 139, 1831

Schlegel, D. J., Finkbeiner, D. P., \& Davis, M. 1998, ApJ, 500, 525

Schlegel, E. M., Schaefer, B., Pagnotta, A., et al. 2010a, ATel, 2419, 1

Schlegel, E. M., Schaefer, B., Pagnotta, A., et al. 2010b, ATel, 2430, 1

Schwarz, G., Woodward, C., Starrfield, S., et al. 2007a, BAAS, 38, 99

Schwarz, G. J., Hauschildt, P. H., Starrfield, S., et al. 1998, MNRAS, 300, 931

Schwarz, G. J., Ness, J.-U., Osborne, J. P., et al. 2008, ATel, 1847, 1

Schwarz, G. J., Osborne, J. P., Page, K. L., et al. 2009a, ATel, 2157, 1

Schwarz, G. J., Osborne, J. P., Page, K. L., et al. 2009b, ATel, 2173, 1

Schwarz, G. J., Osborne, J. P., Page, K. L., et al. 2010, ATel, 2904, 1

Schwarz, G. J., Shore, S. N., Starrfield, S., \& Vanlandingham, K. M. 2007b, ApJ, 657,453

Schwarz, G. J., Shore, S. N., Starrfield, S., et al. 2001, MNRAS, 320, 103

Senziani, F., Skinner, G. K., Jean, P., \& Hernanz, M. 2008, A\&A, 485, 223

Shafter, A. W. 1997, ApJ, 487, 226

Shara, M. M., Yaron, O., Prialnik, D., \& Kovetz, A. 2010, ApJ, 712, L143

Shara, M. M., Zurek, D. R., Williams, R. E., et al. 1997, AJ, 114, 258

Shore, S. N. 2008, in Classical Novae, ed. M. Bode \& A. Evans (2nd ed.; Cambridge: Cambridge Univ. Press), 194

Shore, S. N., \& Gehrz, R. D. 2004, A\&A, 417, 695

Shore, S. N., Genovali, K., \& Wahlgren, G. M. 2011, arXiv:1109.5397

Shore, S. N., Sonneborn, G., Starrfield, S., Gonzalez-Riestra, R., \& Polidan, R. S. 1994, ApJ, 421, 344

Shore, S. N., Starrfield, S., \& Sonneborn, G. 1996, ApJ, 463, L21

Shore, S. N., Wahlgren, G. M., Augusteijn, T., et al. 2011, A\&A, 527, A98

Sitko, M., Lynch, D. K., Russell, R. W., Rudy, R. J., \& Woodward, C. E. 2008, IAU Circ., 8998, 3

Siviero, A., Munari, U., \& Jones, A. F. 2005, IBVS, 5638, 1

Siviero, A., Munari, U., Valentini, M., \& Valisa, P. 2006, IAU Circ., 8702, 2

Snijders, M. A. J., Cassatella, A., Hassall, B. J. M., Harris, A., \& Green, D. W. E. 1985, IAU Circ., 4067, 2

Sokoloski, J. L., Luna, G. J. M., Mukai, K., \& Kenyon, S. J. 2006, Nature, 442, 276

Soma, M., Takao, A., Yamaoka, H., et al. 2005, IAU Circ., 8607, 1
Sostero, G., Yamaoka, H., Kiyota, S., Nakajima, K., \& Maehara, H. 2004, IAU Circ., 8445, 3

Starrfield, S., Iliadis, C., \& Hix, R. 2008, in Classical Novae, ed. M. Bode \& A. Evans (2nd ed.; Cambridge: Cambridge Univ. Press), 77

Starrfield, S., Krautter, J., Ness, J.-U., et al. 2009, in Chandra's First Decade of Discovery, ed. S. Wolk, A. Fruscione, \& D. Swartz (Boston, MA: Chandra X-ray Center), 199 (http://cxc.harvard.edu/ChandraDecade/)

Starrfield, S., Schwarz, G., Truran, J. W., \& Sparks, W. M. 2000, in AIP Conf. Proc. 522 Cosmic Explosions: Tenth Astrophysical Conference, ed. W. W. Zhang \& S. S. Holt (Melville, NY: AIP), 379

Stickland, D. J., Penn, C. J., Seaton, M. J., Snijders, M. A. J., \& Storey, P. J. 1981, MNRAS, 197, 107

Stringfellow, G. S., \& Walter, F. M. 2004, BAAS, 36, 1599

Strope, R. J., Schaefer, B. E., \& Henden, A. A. 2010, AJ, 140, 34

Sugano, M., Kosai, H., Alcock, G. E. D., et al. 1991, IAU Circ., 5222, 1

Suzuki, A., \& Shigeyama, T. 2010, ApJ, 723, L84

Swierczynski, E., Ragan, E., Galan, C., \& Mikolajewski, M. 2008, ATel, 1723, 1

Szkody, P., \& Ingram, D. 1994, ApJ, 420, 830

Takamizawa, K., Kato, T., Yamamoto, M., et al. 2000, IAU Circ., 7409, 1

Terada, Y., Hayashi, T., Ishida, M., et al. 2008, PASJ, 60, 387

Thoroughgood, T. D., Dhillon, V. S., Littlefair, S. P., Marsh, T. R., \& Smith, D. A. 2001, MNRAS, 327, 1323

Tomov, T., Mikolajewski, M., Brozek, T., et al. 2008a, ATel, 1485, 1

Tomov, T., Mikolajewski, M., Ragan, E., Swierczynski, E., \& Wychudzki, P. 2008b, ATel, 1475, 1

Tsujimoto, M., Takei, D., Drake, J. J., Ness, J.-U., \& Kitamoto, S. 2009, PASJ, 61,69

van den Bergh, S., \& Younger, P. F. 1987, A\&AS, 70, 125

Vanlandingham, K. M., Schwarz, G., Starrfield, S., et al. 2007, BAAS, 38, 99

Vanlandingham, K. M., Schwarz, G. J., Shore, S. N., \& Starrfield, S. 2001, AJ, 121,1126

Vanlandingham, K. M., Starrfield, S., Wagner, R. M., Shore, S. N., \& Sonneborn, G. 1996, MNRAS, 282, 563

van Rossum, D. R., \& Ness, J.-U. 2010, Astron. Nachr., 331, 175

Vaytet, N. M. H., O’Brien, T. J., \& Bode, M. F. 2007, ApJ, 665, 654

Waagen, E. O., Granslo, B., Reszelski, M., et al. 2006, CBET, 466, 2

Waagen, E. O., Henden, A., Maury, A., et al. 2008, IAU Circ., 8999, 1

Wagner, R. M., Starrfield, S. G., Rohrbach, J., et al. 2003, IAU Circ., 8176, 3

Walder, R., Folini, D., Favre, J. M., \& Shore, S. N. 2010, in ASP Conf. Ser. 429, Numerical Modeling of Space Plasma Flows, Astronum-2009, ed. N. V. Pogorelov, E. Audit, \& G. P. Zank (San Francisco, CA: ASP), 173

Walder, R., Folini, D., \& Shore, S. N. 2008, A\&A, 484, L9

Walter, F. M. 2007, IAU Circ., 8800, 2

Warner, B. 2008, in Classical Novae, ed. M. Bode \& A. Evans (2nd ed.; Cambridge: Cambridge Univ. Press), 16

Warner, B., \& Woudt, P. A. 2009, MNRAS, 397, 979

Whitelock, P. A., Carter, B. S., Feast, M. W., et al. 1984, MNRAS, 211, 421

Williams, P., Waagen, E. O., Krajci, T., \& Schmeer, P. 2006, IAU Circ., 8700, 1

Williams, R. E., Hamuy, M., Phillips, M. M., et al. 1991, ApJ, 376, 721

Woodward, C. E., Gehrz, R. D., Jones, T. J., \& Lawrence, G. F. 1992, ApJ, 384, L41

Woodward, C. E., \& Starrfield, S. 2011, Can. J. Phys., 89, 333

Woudt, P. A., \& Warner, B. 2001, MNRAS, 328, 159

Woudt, P. A., \& Warner, B. 2003, MNRAS, 343, 313

Yamaoka, H., Nakamura, Y., Itagaki, K., Nakano, S., \& Nishimura, H. 2007a, IAU Circ., 8810, 1

Yamaoka, H., Waagen, E., Amorim, A., et al. 2007b, IAU Circ., 8807, 1

Yaron, O., Prialnik, D., Shara, M. M., \& Kovetz, A. 2005, ApJ, 623, 398 